\title{
Petroleum Potentials of the Nigerian Benue Trough and Anambra Basin: A Regional Synthesis
}

\section{B. Abubakar}

National Centre for Petroleum Research and Development, Abubakar Tafawa Balewa University, Bauchi, Nigeria. Email: ambello2002@yahoo.com, ambello@ncprd.org.ng

Received October $20^{\text {th }}, 2013$; revised November $23^{\text {rd }}, 2013$; accepted December $14^{\text {th }}, 2013$

Copyright (c) 2014 M. B. Abubakar. This is an open access article distributed under the Creative Commons Attribution License, which permits unrestricted use, distribution, and reproduction in any medium, provided the original work is properly cited. In accordance of the Creative Commons Attribution License all Copyrights (c) 2014 are reserved for SCIRP and the owner of the intellectual property M. B. Abubakar. All Copyright (C) 2014 are guarded by law and by SCIRP as a guardian.

\section{ABSTRACT}

A review on the geology and petroleum potentials of the Nigerian Benue Trough and Anambra Basin is done to identify potential petroleum systems in the basins. The tectonic, stratigraphic and organic geochemical evaluations of these basins suggest the similarity with the contiguous basins of Chad and Niger Republics and Sudan, where commercial oil discovery have been made. At least two potential petroleum systems may be presented in the basins: the Lower Cretaceous petroleum system likely capable of both oil and gas generation and the Upper Cretaceous petroleum system that could be mainly gas-generating. These systems are closely correlative in temporal disposition, structures, source and reservoir rocks and perhaps generation mechanism to what obtains in the Muglad Basin of Sudan and Termit Basin of Niger and Chad Republics. They are very effective in planning future exploration campaigns in the basins.

\section{KEYWORDS}

Benue Trough; Anambra Basin; Petroleum Potentials; Southern Benue Trough; Central Benue Trough; Northern Benue Trough

\section{Introduction}

Petroleum (oil and gas) accounts for up to 95\% of the Nigeria's foreign earnings [1,2] and has remained the major supporter of its economy since it was first discovered in commercial volume in 1956. Globally, petroleum as an energy source will continue to dominate other primary energy sources and is expected to account for up to $56 \%$ of the world energy demand in the year 2030. Therefore, it is expected that a review on the petroleum potentials of the Nigerian Benue Trough and Anambra Basin (Figure 1(a)) will provide the necessary impetus for exploration activities in these frontier inland basins. This paper attempts to synthesize the results of multiple researches done in the basins over the years for easy reference and effective understanding. The paper has attempted to identify potential petroleum system elements in the basins and the tectonic processes related to trap formation and generation.

\section{Geologic and Tectonic Setting}

The Benue Trough of Nigeria (Figure 1(a)) is an intracontinental basin in Central West Africa that extends NE to SW. It is over $1000 \mathrm{~km}$ in length and exceeds $150 \mathrm{~km}$ in width. Its southern outcrop limit is the northern boundary of the Niger Delta Basin, while the northern outcropping limit is the southern boundary of the Chad Basin separated from the Benue Trough by an anticlinal structure termed the "Dumbulwa-Bage High" [3]. The Benue Trough is filled with up to $6000 \mathrm{~m}$ of Cretaceous sediments associated with some volcanics. It is part of a mega-rift system termed the West and Central Africa Rift System (WCARS). The WCARS includes the Termit Basin of Niger and western Chad, the Bongor, Doba and Doseo Basins of southern Chad, the Salamat Basin of Central African Republic and the Muglad Basin of Sudan (Figure 1(b)).

The Benue Trough is geographically subdivided into 


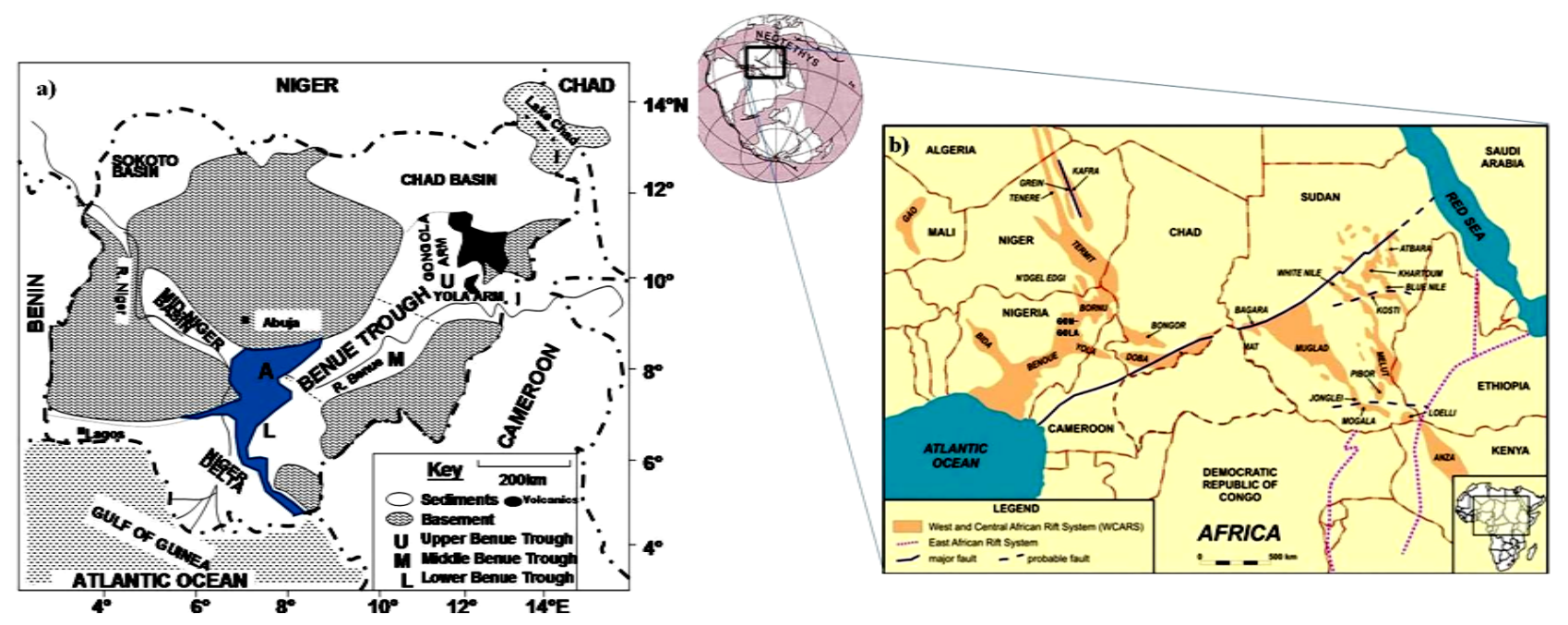

Figure 1. (a) Generalized geological map of Nigeria showing the Benue Trough, blue area represents the Anambra Basin; (b) WCARS showing the Benue Trough (from United Reef Limited Report, 2004). NB: The Anambra Basin is a separate sedimentary basin.

southern, central and northern parts (Figure 1(a)). The origin and tectonic history of the Benue Trough and indeed the entire WCARS is associated with the separation of Africa and South America (break-up of Gondwanaland) during the early Cretaceous time [4]. This break-up was followed by the drifting apart of these continents, the opening of the South Atlantic and the growth of the MidAtlantic ridge [4].

The mechanism responsible for the origin and evolution of the Benue Trough is controversial. However, there are basically two models: the rift system models and the pull-apart model.

Several mechanisms have been proposed based on the rift system. The trough is considered to be a third arm of a triple junction beneath the present Niger Delta. The earliest model was given by [5] and subsequently supported by [6-8] who proposed tensional movements resulting in a rift as the controlling factor. They interpreted an observed axial zone of positive gravity anomalies flanked by linear negative anomalies on both sides as an arrangement typical of rift valleys in general, and resulted from crustal thinning and elevation of crust-mantle boundary beneath the central parts of the rift. The problem with this model, however, is the lack of conspicuous rift faults at the margins of the trough $[9,10]$ and a generalized folding of the Cretaceous sediments. Also, except in the Abakaliki area, Cretaceous magmatic activity associated with rift structures is very scarce; it is only found close to, or along major faults. Reference [7] however, argued that the main boundary rift faults are now concealed by the Cretaceous sediments overlying the margins of the trough. Reference [11] suggested the existence below the Niger Delta and the Southern Benue Trough of a triple junction of the RRR type which indicates the existence of a spreading ridge active from Albian to Santonian (Figure 2(a)). An unstable RRF triple junction model leading to plate dilation and the opening of the Gulf of Guinea was proposed by reference [12] (Figure 2(c)). References $[13,14]$ considered the Benue Trough as the third failed arm (or aulacogen) of a three-armed rift system related to the development of hot spots (Figure 2(b)).

Most recent models based on pull-apart system revealed that wrenching was a dominant tectonic process during the Benue Trough evolution. References [4] and [15] defined the Benue Trough as a set of juxtaposed pull-apart basins generated along pre-existing $\mathrm{N}^{\circ} 0^{\circ} \mathrm{E}$ strike-slip faults during the Lower Cretaceous. The strike-slip (transcurrent) faults are believed to be connected to the oceanic fracture zones and reactivated during the separation of the South American and African plates (Figure 3). This model originated from the fact that most of the major faults identified in the Benue Trough are transcurrent faults rather than normal faults of rift systems. Identified normal faults (mostly $\mathrm{N} 120^{\circ} \mathrm{E}$ trending) in the Benue Trough are seen to control the grabens but are always linked to major sinistral $N 60^{\circ} \mathrm{E}$ strike-slip faults (Figure 4).

During the mid-Santonian, $\mathrm{N} 155^{\circ} \mathrm{E}$ trending compression reactivated the sinistral faults as reverse faults, while the $\mathrm{N} 120^{\circ} \mathrm{E}$ normal fractures acted as dextral strike-slip faults. In the Northern Benue Trough however, there is a controversy as to the existence of the Santonian event. Reference [16] favour the Maastrichtian compression as the only Late Cretaceous event that affected the Northern Benue Trough while several workers such as $[6,17]$ and $[18,19]$ suggested the presence of both the mid-Santonian 


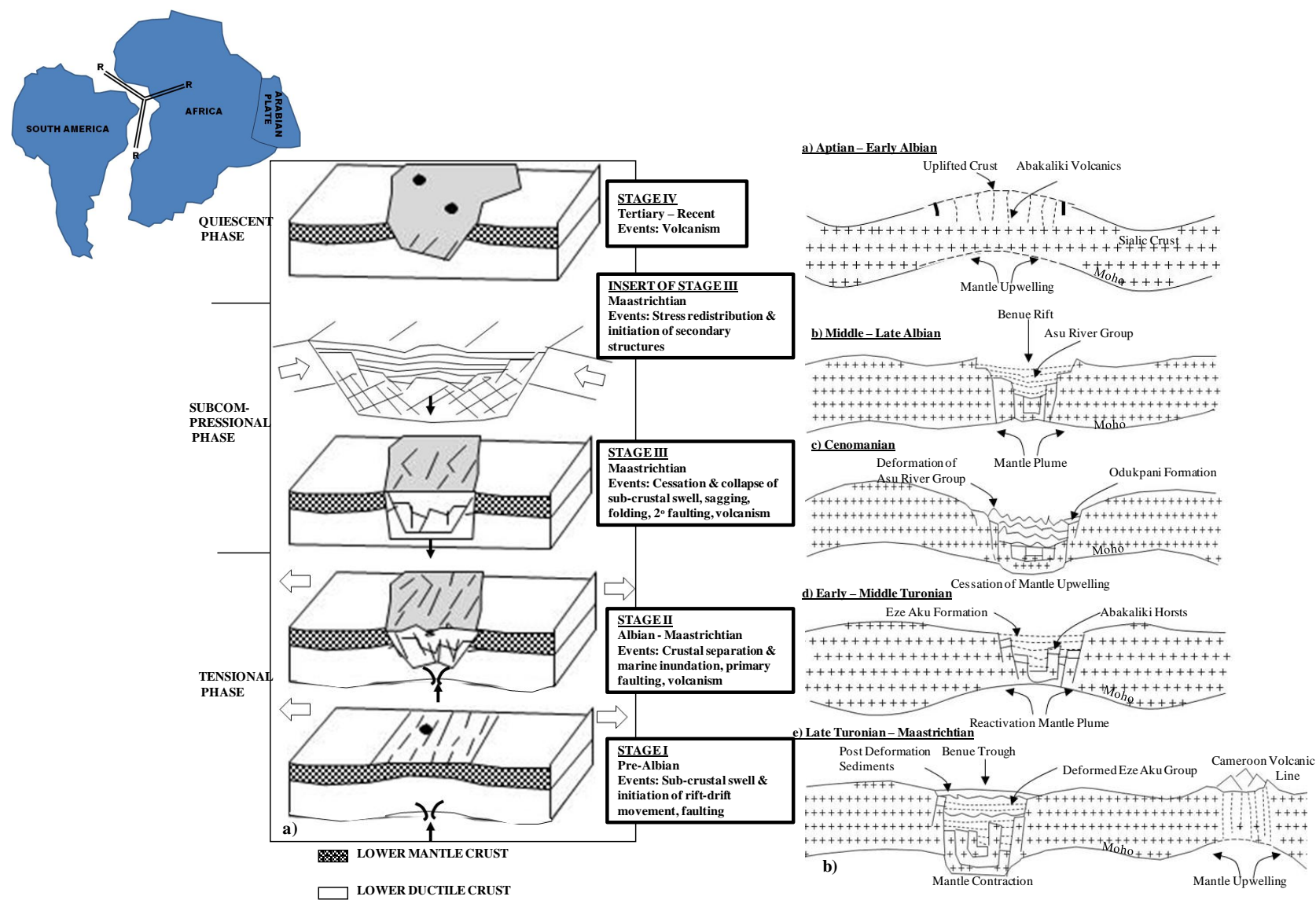

- volCANics

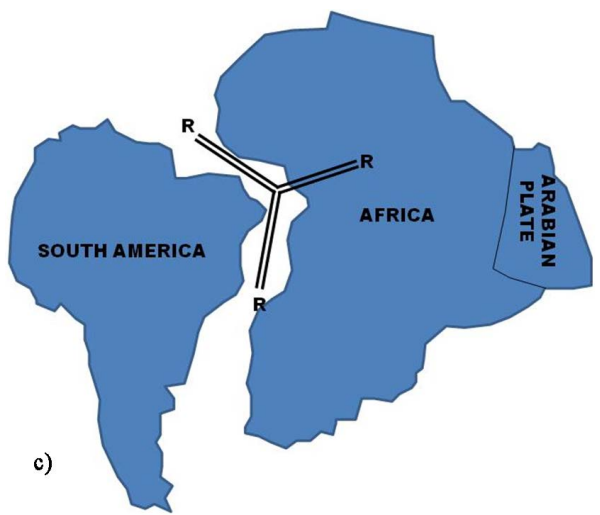

Figure 2. Models for the RRR Rift origin of the Benue Trough of Nigeria. (a) Spreading Ridge Model (from Avbovbo et al. 1986); (b) Aulacogen Model (from Olade, 1975); (c) RRF Model.

and the Maastrichtian events in the trough.

\section{Stratigraphy}

Detailed stratigraphic description of sediments in the Benue Trough was presented by [18-26] amongst many others.

The stratigraphic succession in the Benue Trough is shown in Figure 5. The Albian Asu River Group comprises the oldest marine deposits in the Southern Benue Trough. It includes the shales, limestones and sandstones of the Abakaliki Shale, correlative to the Mfamosing Limestone on the Calabar Flank [23,27]. In the Central Benue Trough, fossiliferous Arufu, Uomba and Gboko Formations are lateral equivalents. Albian sediments (late Aptian-early Cenomanian and older $[28,29])$ in the Northern Benue Trough are represented by the alluvial fan to lacustrine to fluvial Bima Formation.

There was virtually no deposition during the Cenomanian in the Southern Benue Trough except the Odukpani Formation in the Calabar flank [22]. The period is be- 


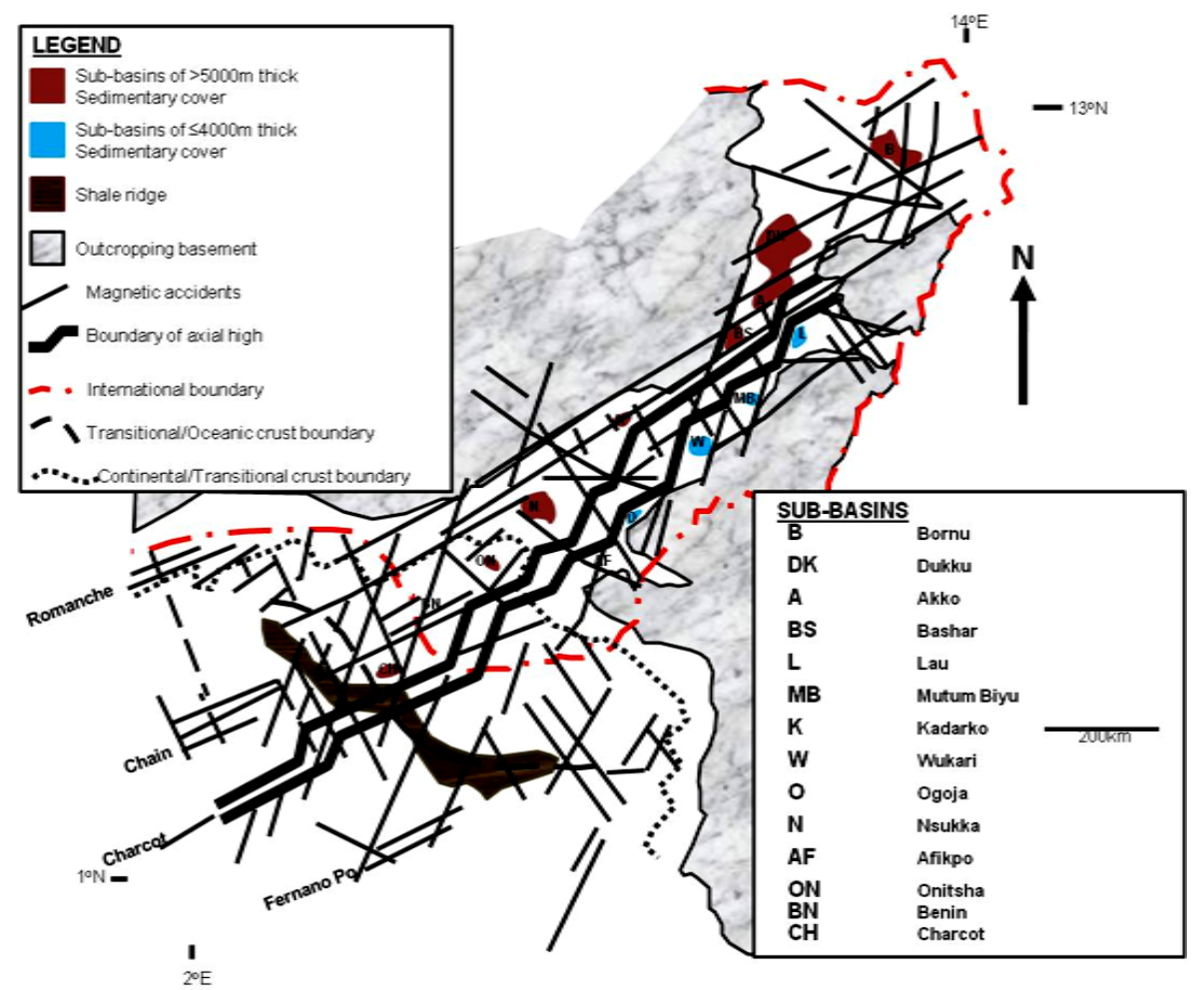

Figure 3. Magnetic discontinuities showing axially placed structural high associated with transcurrent faulting and location of some sub-basins in the Chad Basin, Benue Trough, Anambra Basin, Niger Delta and Nigerian Continental Margin (from Benkhelil et al., 1989).

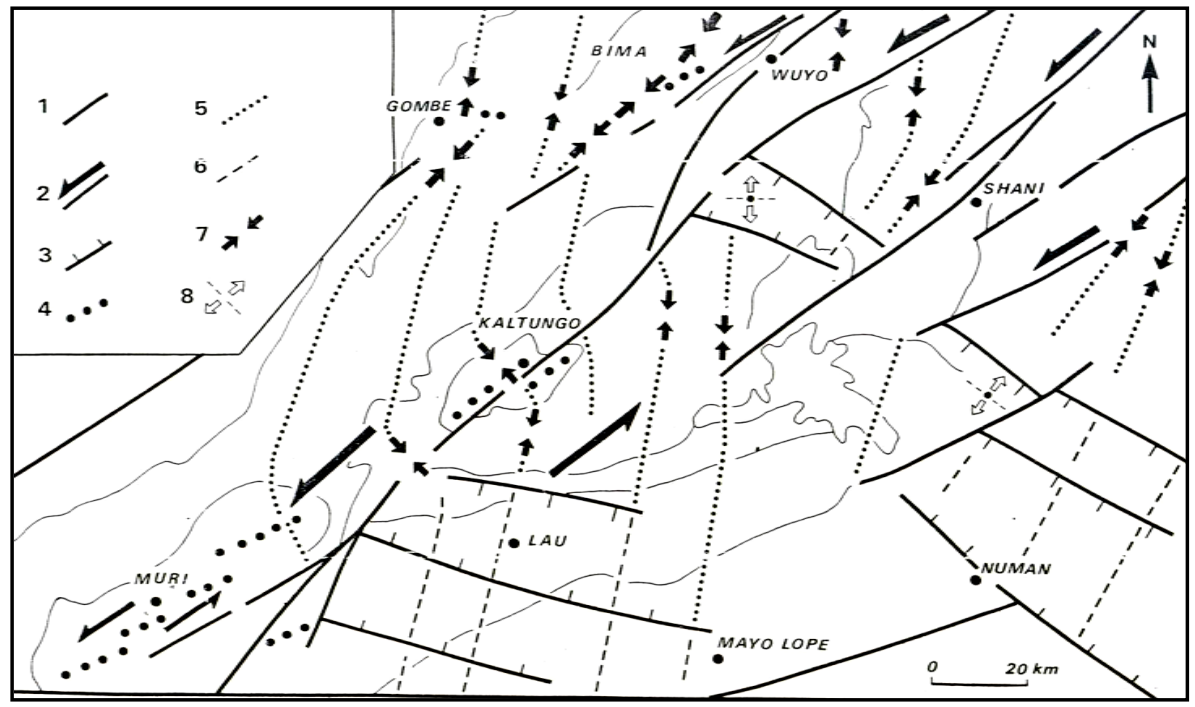

Figure 4. Microtectonic analysis of structural setting of the Early Cretaceous in the Northern Benue Trough. 1, regional fault; 2, sinistral fault; 3 , normal fault; 4 , anticline axis; $5, \delta_{1}$ trajectories; $6, \delta_{3}$ trajectories; 7 , compressive strike-slip tensors; 8 , extensional tensors (from Guiraud, 1990).

lieved to represent minor regression in the Southern and the Central Benue Trough [30] perhaps caused by a compressional event [31,32]. In the Central Benue Trough, the regressive Awe and Keana Formations were deposited [33] while in the Northern Benue Trough; the transitional Yolde Formation marked the Cenomanian [34].
The late Cenomanian to early Turonian was a period of major transgression throughout the Benue Trough that culminated into possible link between the waters of the Gulf of Guinea to the south and the Tethys Sea to the north [18]. In the Southern Benue Trough, the Ezeaku Group which comprises the Nkalagu Formation (black 


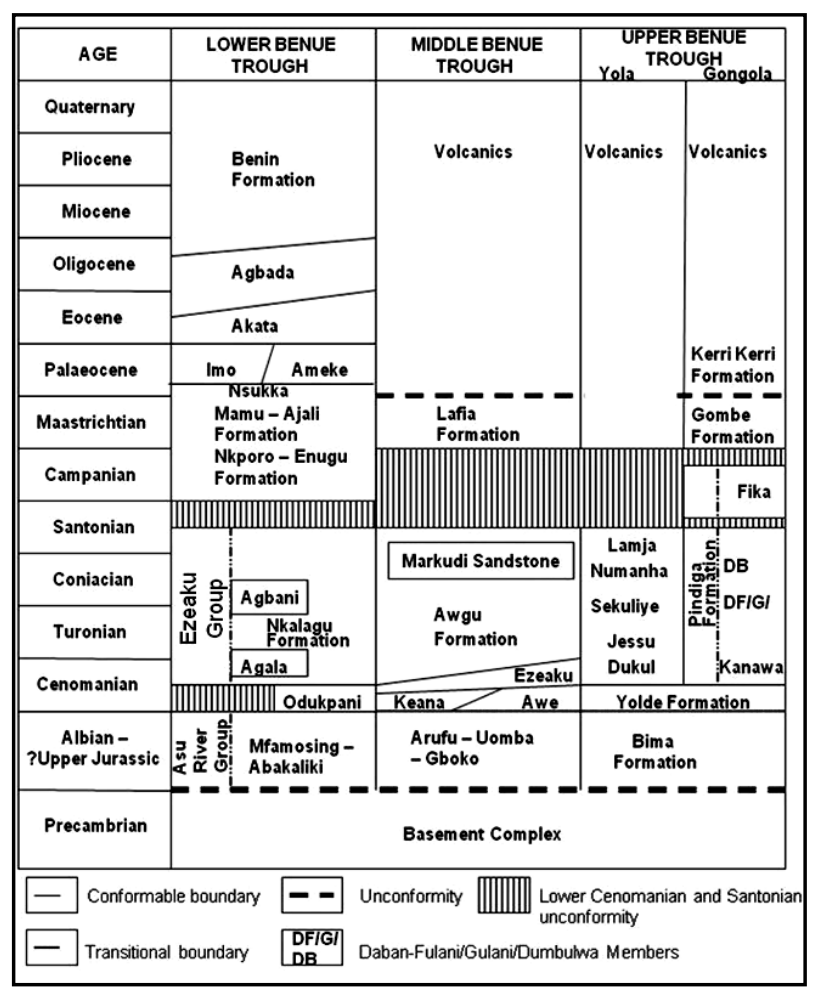

Figure 5. Microtectonic analysis of structural setting of the Early Cretaceous in the Northern Benue Trough. 1, regional fault; 2 , sinistral fault; 3 , normal fault; 4 , anticline axis; 5 , $\delta_{1}$ trajectories; $6, \delta_{3}$ trajectories; 7 , compressive strike-slip tensors; 8, extensional tensors (from Guiraud, 1990).

shales, limestones and siltstones) and interfingering regressive sandstones of the Agala and Agbani Formations were deposited up to the Santonian [23]. In the Central Benue Trough, the marine facies of the Ezeaku Group and the Awgu Formation are the lateral equivalents. In the Gongola Sub-basin of the Northern Benue Trough, the Pindiga Formation [6], represented by the marine Kanawa Member, the regressive fluvial and littoral sandy facies of the Gulani, the Dumbulwa and the Deba Fulani (Daban Fulani) Members [3] were deposited. The limestones and shales of the Dukul Formation, the mudstones of the Jessu Formation, the Sekuliye Formation, the Numanha Shale and the Lamja Sandstone (all of the Yola Sub-basin of the Northern Benue Trough) are the facies equivalents of the Pindiga Formation. The Fika Shale [6] in the Gongola Sub-basin which is also equivalent to the Fika Member [3] was correlated to be part of the late Cenomanian to early Santonian sequence [24] but biostratigraphic studies by $[34,35]$, and recent observations on field relationship indicate that this formation is postfolding Campano-Maastrichtian deposit.

During the mid-Santonian period, all the pre-Santonian sediments were folded and uplifted as a result of global plate reorganization [36]. The Abakaliki area was folded intensely into Abakaliki anticlinorium accompanied by minor igneous activity. This resulted in the formation of depression on either flank of the anticlinorium: the small Afikpo syncline on the southeast and the wider Anambra Basin on the northwest. In the Southern Benue Trough initial transgression followed by a regressional period started after the mid-Santonian folding, and the Anambra Basin became the new depocenter where CampanoMaastrichtian shales of the Enugu and the Nkporo Formations, coal measures of the Mamu Formation, and fluvio-deltaic sandstones of the Ajali Formation were deposited. The regressional period marked the beginning of a proto-Niger Delta [37]. In the Central Benue Trough, the fluvio-deltaic Lafia Formation represents the only lateral facies equivalent of the post-Santonian sediments. In the Northern Benue Trough, the Gombe Formation, a Maastrichtian sediment, overlies the Campano-Maastrictian Fika Shale.

Tertiary sediments (debatably considered not part of the Benue Trough by [25]) were restricted to the western part of the Northern Benue Trough where the continental Kerri-Kerri Formation unconformably overlies the Gombe Formation $[3,6]$. Tertiary sediments are not recorded in the Central Benue Trough. In the Southern Benue Trough, there was a major transgression in the $\mathrm{Pa}-$ laeocene $[18,33]$ terminating the advance of the Upper Cretaceous proto-Niger Delta. Sedimentation was restricted to the Anambra Basin where the Imo Shale and the Ameki Formation together with their sub-surface equivalent (the Akata and the Agbada Formations) were deposited.

\section{Tectonic Structures}

Tectonic and structural development of the Benue Trough and Anambra Basin is related to their origin and evolution and on regional scale comparable to what obtains in other basins of the WCARS.

Generally, basins within the WCARS are divided into two subsystems: the NW-SE trending West African rift sub-system (WARS) mostly situated in Niger Republic (e.g. Termit Basin) and the E-W trending Central African rift sub-system (CARS) that includes basins of the southern Chad Republic, Salamat Basin of the Central African Republic and the Sudanese basins [38] (Figure 1(b)). While the WARS basins are characteristically rift basins (half-grabens), the CARS counterparts were strongly affected by strike-slip (transcurrent) faulting associated with the Central African Shear Zone (CASZ) (Figure 1(b)). In this classification, the Benue Trough and the post-Santonian evolved Anambra Basin of Nigeria are considered part of the WARS, representing southwestern extension of the Termit Basin of Niger into Nigeria. But as pointed earlier from the works of [4] and [15], the Benue Trough was strongly affected by transcurrent faulting (Figures 3, 6) at different times of its evolutio- 


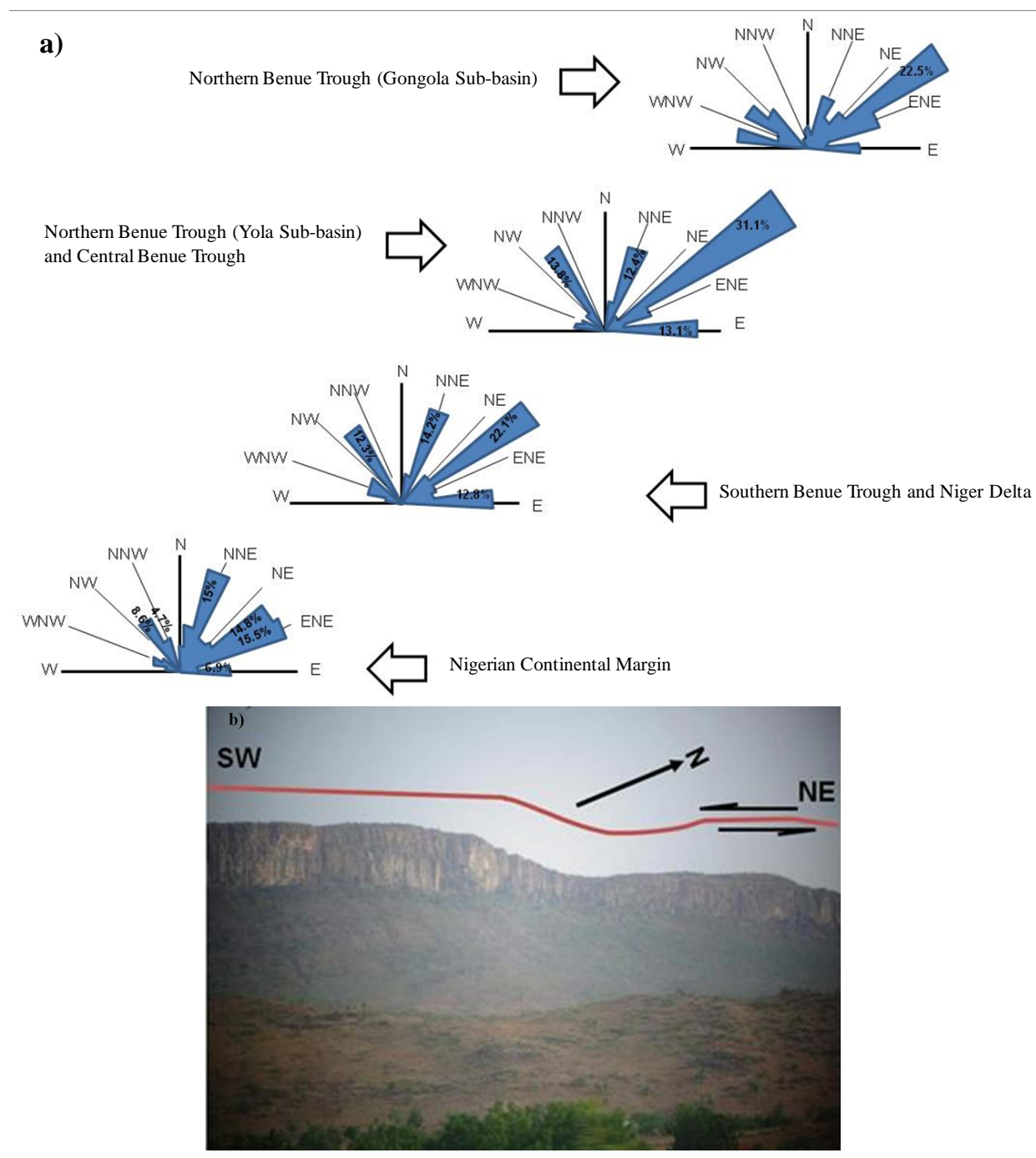

Figure 6. (a) Frequency diagrams of magnetic discontinuities showing major structural trends in the Benue Trough through to the Nigerian continental margin (after Benkhelil et al., 1989), (b) NE-SW trending sinistral strike-slip fault at Bima Hill, Northern Benue Trough.

nary history such that it developed strong similarity with the Doba and Bongor Basins of the CARS. Therefore, the Benue Trough may be structurally more complex and is expected to develop structures peculiar to both the WARS and CARS. The close proximity of the Anambra Basin to the Niger Delta and their seemingly related basins development make also possible the presence of structural elements in the Anambra Basin similar to those in the onshore Niger Delta.

The structural and stratigraphic framework of the WCARS, to which the Benue Trough and Anambra Basin belongs, owes its origin to three major rift phases and two non-rift phases (Figure 7) of:

1) Post-Rift Phase (Miocene-Recent);

2) Palaeogene Rift Phase III (Palaeocene-Oligocene);

3) Upper Cretaceous Rift Phase II (Late CenomanianMaastrichtian);
4) Lower Cretaceous Rift Phase I (Late Jurassic-Albian);

5) Pre-Rift Phase (as Late Jurassic).

See [38] for details. For simplicity of purpose in this review however, the structurations in the Benue Trough and Anambra Basin and by extension the WCARS can be grouped into three:

1) Post Cretaceous Structuration;

2) Late Cretaceous Structuration and Inversion;

3) Early Cretaceous Structuration.

The subsequent discussion of these groups utilizes the works of $[3,15,17,38,39]$ and field experience.

\subsection{The Early Cretaceous Structuration}

The Early Cretaceous structurations are related to Neocomian-Albian rifting phase (Lower Cretaceous Rift

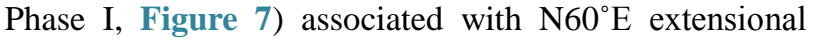




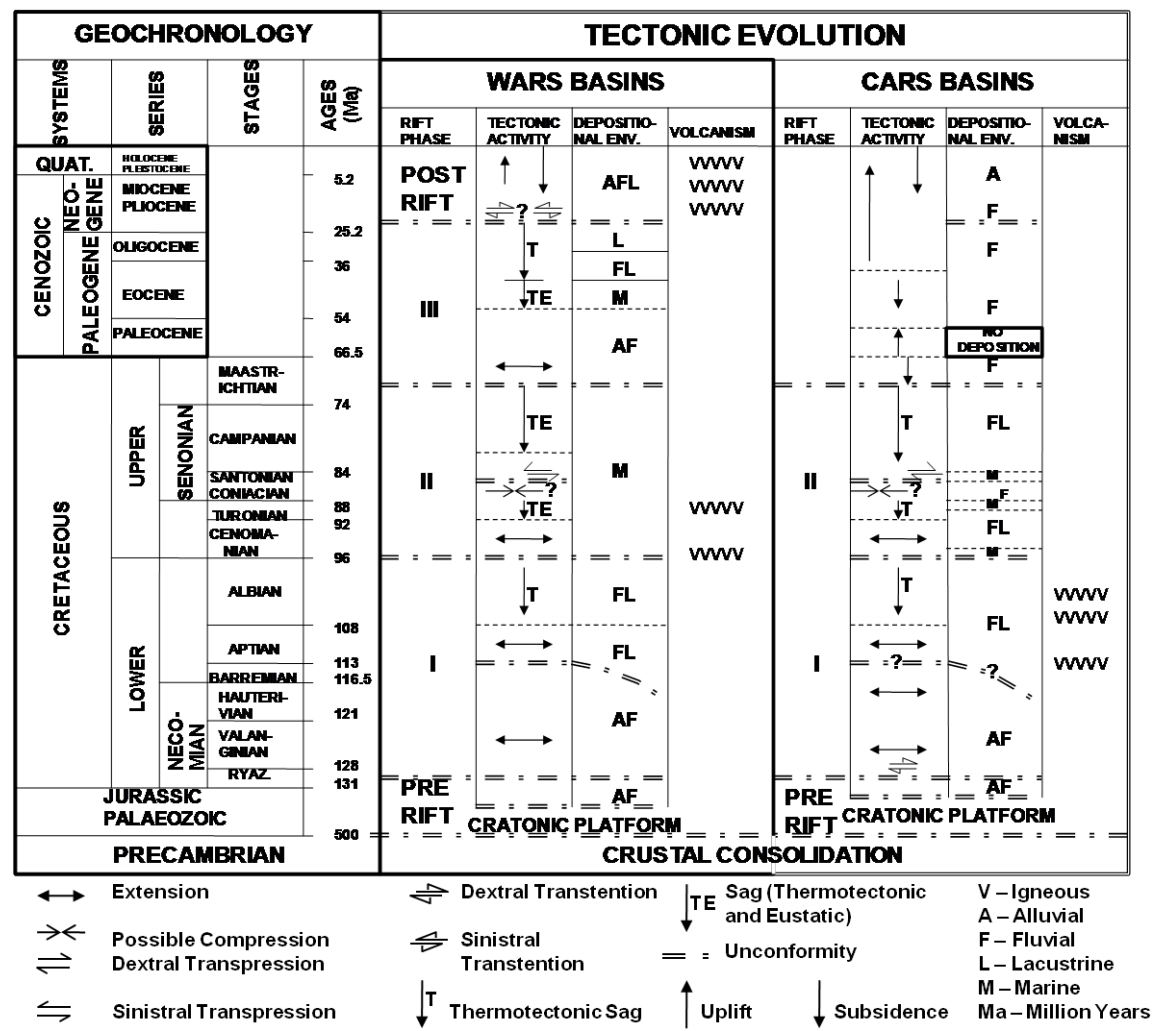

Figure 7. Tectonic Framework of the WCARS basins (including the Benue Trough and the Anambra Basin) (modified from Genik, 1993).

regimes. The resulting structural elements seem to be closely related to sinistrally re-activated transcurrent faulting. Structures include:

1) Mainly sinistral strike-slip faults with dominant N50 - $70^{\circ} \mathrm{E}$ trends (Figure 6);

2) $\mathrm{N} 120-130^{\circ} \mathrm{E}$ normal faults associated with transtensional and pure extensional stress regimes forming fault blocks (Figure 4). The normal faults control the grabens but always linked to major sinistral $\mathrm{N} 60^{\circ} \mathrm{E}$ strike-slip faults;

3) Locally associated large scale drag folds (e.g. Bima anticline, Figure 8) with sub-parallel axial traces to major NE-SW wrench faults;

4) Horsts and grabens (Figure 9);

5) Regional unconformities (Barremian and upper Aptian unconformities in the Northern Benue Trough, Figure 10(a), and upper Albian unconformity in the southern Benue Trough. Note: upper Albian regional unconformity is questionable in the Northern Benue Trough).

These structures were buried/sealed by the Upper Cretaceous sedimentation related to the Upper Cretaceous Rift Phase II (Figure 10(b)).

\subsection{The Late Cretaceous Structuration and Inversion}

The Late Cretaceous structurations are related to the San-

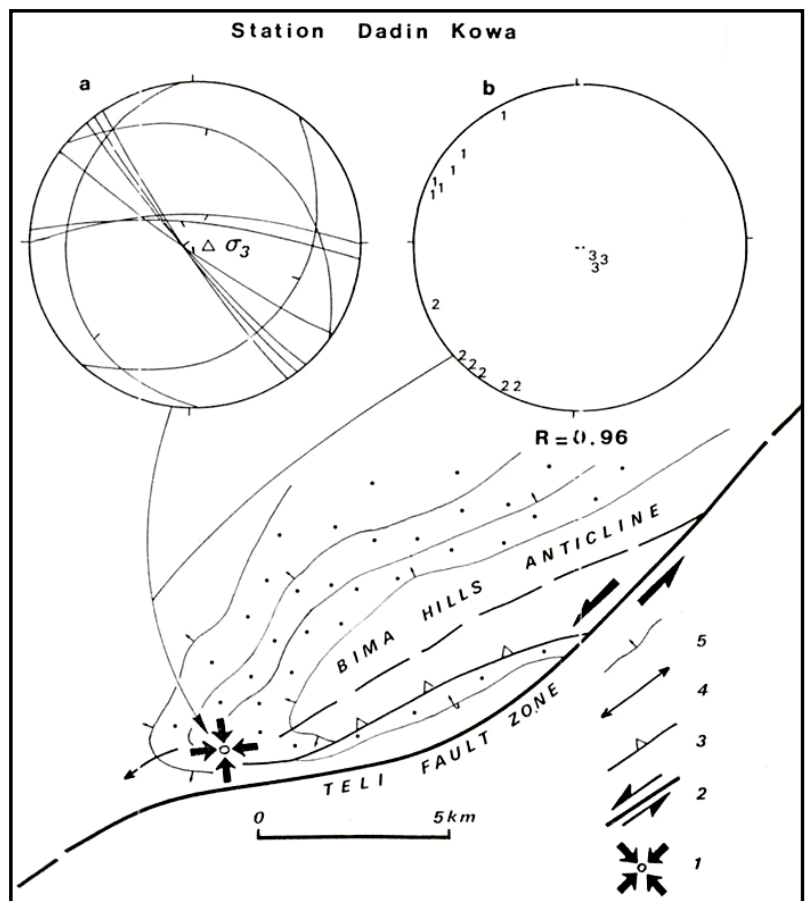

Figure 8. Structural setting of Bima Hill, Northern Benue Trough showing the Bima anticline. 1, tensor of vertically axial symmetric extension; 2 , sinistral wrench fault; 3 , reverse fault; 4, anticline axis; 5, bedding trace (from Guiraud et al., 1993). 


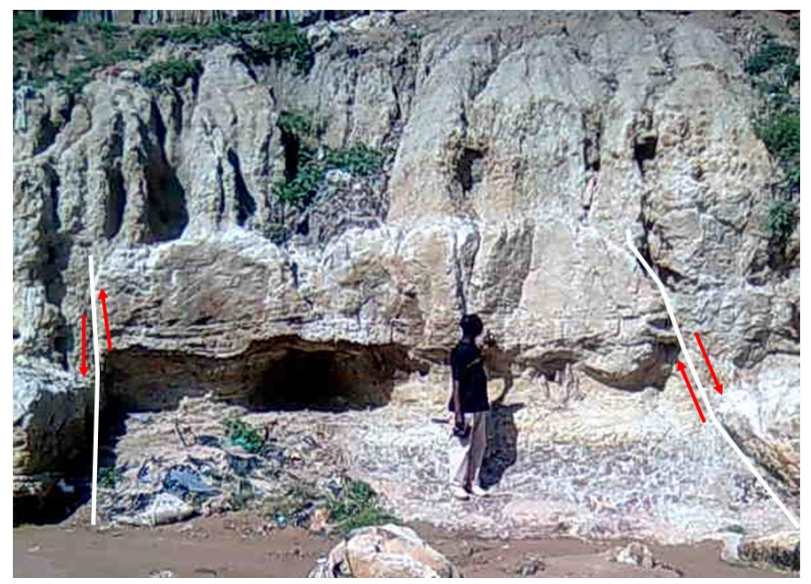

Figure 9. Small horst and graven structure in the Northern Benue Trough.

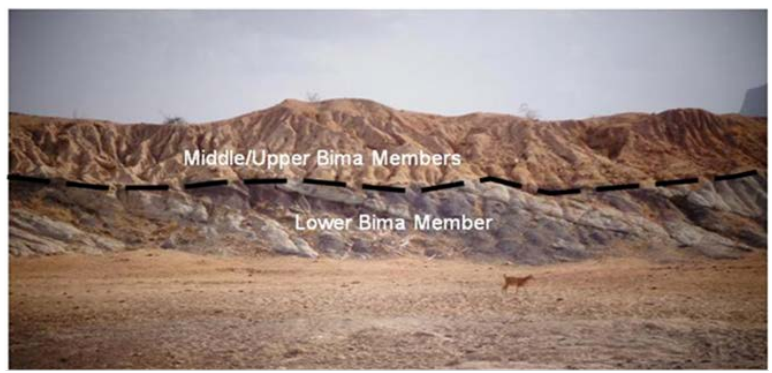

(a)

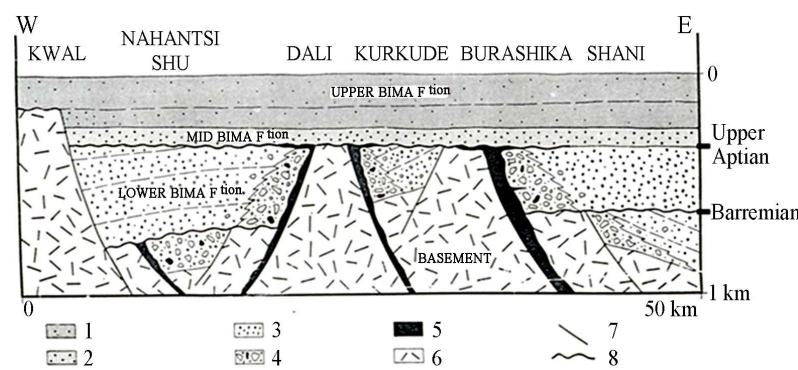

(b)

Figure 10. Small horst and graven structure in the Northern Benue Trough.

tonian $\mathrm{N} 155^{\circ} \mathrm{E}$ trending compression and transtensional inversion in the Southern and Central Benue Troughs, and Santonian/Maastrichtian compressions in the Northern Benue Trough. The structural elements formed seem to be closely related to transpressional processes associated with the reactivation of the Early Cretaceous N50 $70^{\circ} \mathrm{E}$ sinistral strike-slip faults mainly as reverse faults (e.g. Bima-Teli fault zone, Figure 11(a)), and the N120 $130^{\circ} \mathrm{E}$ normal faults as dextral strike-slip faults (Figures 11(c), (d)). Generated structures include:

1) Large-scale NE-SW trending transpressional anticlines (Figure 11(a));

2) Drag folds;

3) Flower structures (Figure 11(b));
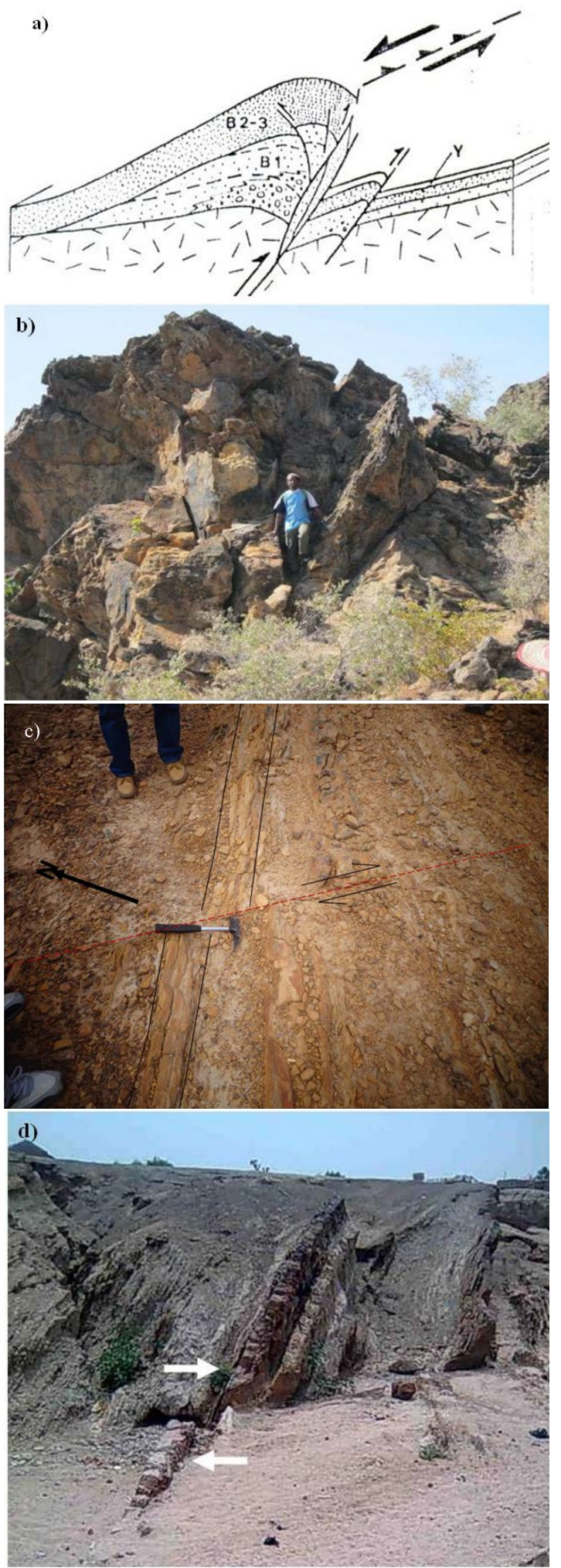

Figure 11. Late Cretaceous structures from the Northern Benue Trough. (a) Schematic diagram of the Bima Hill traspressional anticline from Guiraud, 1993 related to the Santonian/Maastrichtian events; (b) Flower structure petals (an evidence of basin inversion); (d), (c) NW-SE trending dextral strike-slip faults. 
4) Inverted fault blocks;

5) E-W and NW-SE trending dextral strike-slip faults (Figures 11(c), (d)).

Post Santonian to Palaeocene sedimentation located towards the NW portion of the Benue Trough (e.g. the Anambra Basin in the Southern Benue Trough and the Kerri-Kerri Sub-basin in the Northern Benue Trough) truncates and sealed the structures of the Late Cretaceous Inversion events (Figure 12).

The "Santonian event" had wide-ranging effects significant for hydrocarbon exploration in the Benue Trough and the entire WCARS. It created hydrocarbon trapping folds in southern WARS and CARS [38], folded the Benue Trough [40,4], and produced hydrocarbon trapping folds in the Muglad Basin of Sudan [41].

Related to the Late Cretaceous tectonics are identified field-scale growth faults and roll-over anticlines in the deltaic Enugu Formation in the Anambra Basin and Gombe Formation in the Gongola Basin of the Northern Benue Trough (Figure 13).

\subsection{The Post Cretaceous Structuration}

Although references [39,42-44] reported cases of few post-Cretaceous structurations in the Kerri-Kerri Subbasin and the Anambra Basin (considered not part of the Benue Trough by [25]); this time interval was mainly a quiescence period. The identified structures are mainly normal faults perhaps related to the Palaeogene Rift
Phase III (Figure 7).

\section{Petroleum Potentials/Possible Petroleum Systems}

The origin of the Benue Trough and Anambra Basin has been shown to be related to rifting and basin inversion respectively. Basins formed as rifts have high geothermal gradients and large traps for hydrocarbons [40]. Reference [45] showed that $35 \%$ of rifted basins contain giant oil fields. The discovery of oil in the contiguous basins of Niger, Chad and Sudan, the discovery of the 33BCF of gas in well Kolmani River-1 in the Gongola Sub-basin [46], and oil/gas in some exploratory wells in the Anambra Basin [47] attest to the presence of petroleum system(s) in both the Benue Trough and the Anambra basin.

Petroleum system concept describes the genetic relationship between a pod of active source rock and the resulting oil and gas accumulations and encompasses four essential elements of source rock, reservoir rock, seal rock and overburden, and two processes of trap formation and generation/migration/accumulation [48].

As part of the WCARS, it is instructive to evaluate the petroleum potentials of the Benue Trough/Anambra Basin within the context of the identified petroleum systems in the WCARS. In both the Benue Trough and Anambra Basin, sediment thickness is in excess of $4000 \mathrm{~m}$ [39]. This is more than the minimum overburden thickness of $1000 \mathrm{~m}$ [49] required for a basin to be prospective if all other elements of a petroleum system are present.
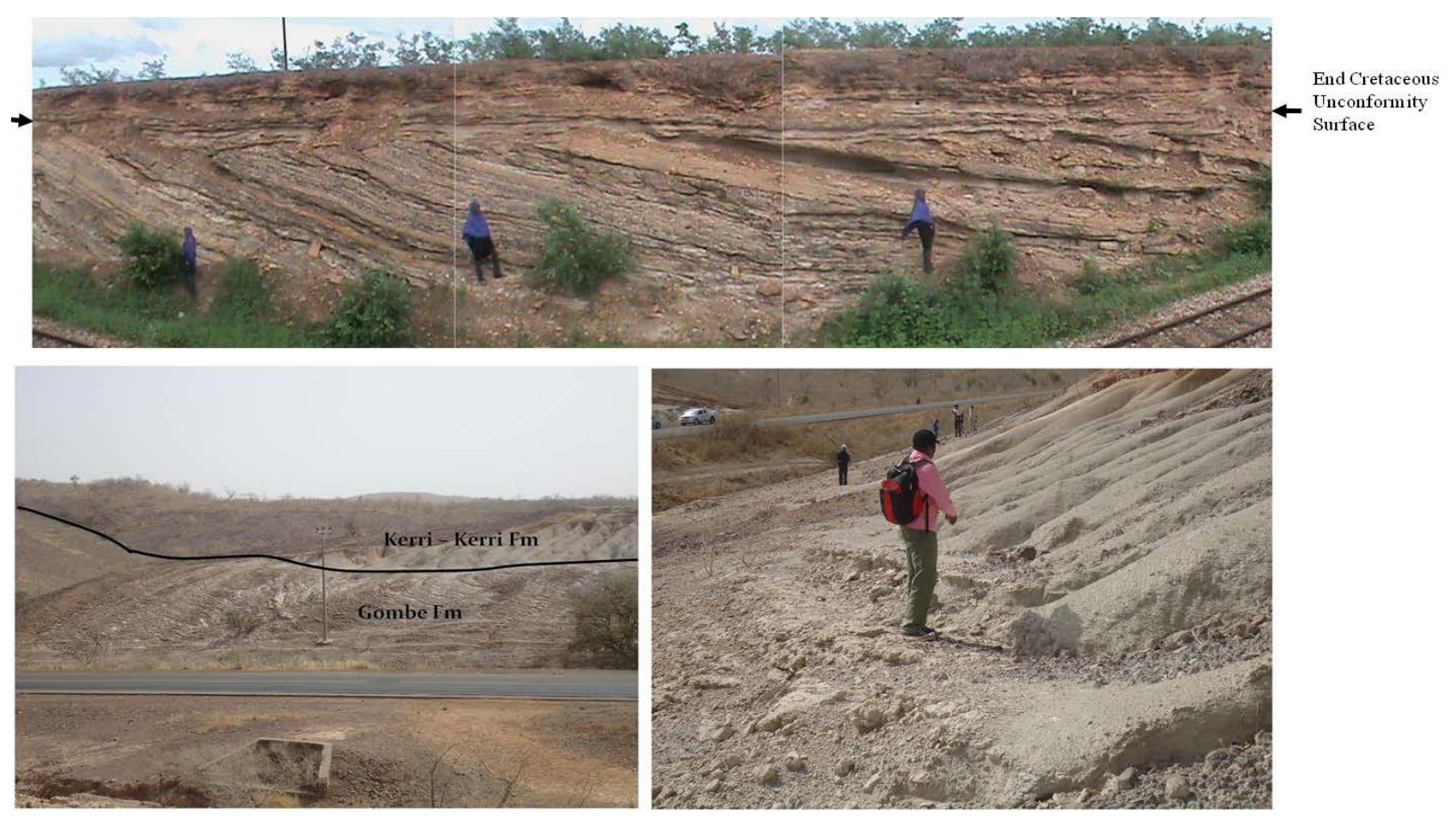

Figure 12. Various expressions of late cretaceous angular unconformities in Northern Benue Trough. 

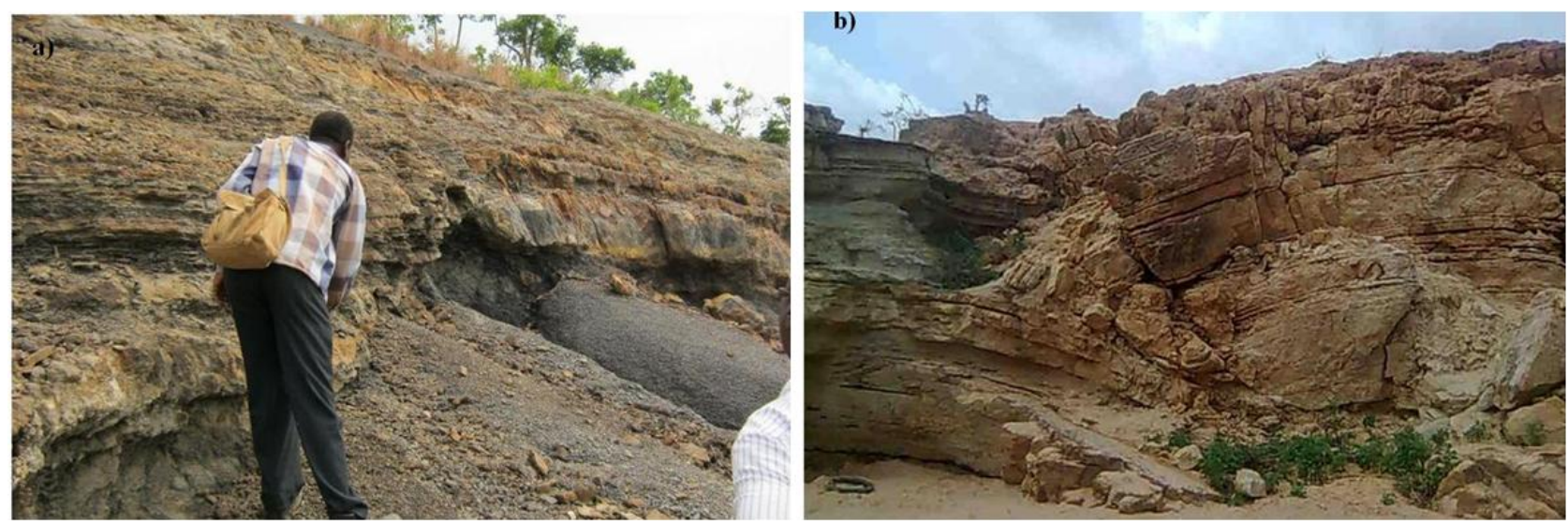

Figure 13. (a) Growth fault in the Enugu formation, Anambra Basin; (b) Small-scale growth fault and roll-over anticline in the Gombe formation, Gongola sub-basin, Northern Benue Trough.

\subsection{Petroleum Systems}

Three petroleum systems can be identified in the WCARS basins [38]. Reference [50] also recognized same distinct hydrocarbon systems in the southern Nigeria onshore and offshore basins albeit with very minor modification. These systems are related to the three major rift phases that affected the WCARS (Figure 7), hence mostly individually confined within the identified sequence bounded assemblages of the rift phases.

The identified petroleum systems are:

1) Lower Cretaceous Petroleum System (including the most basal Upper Cretaceous "Cenomanian”);

2) Upper Cretaceous Petroleum System;

3) Palaeogene Petroleum System.

\subsubsection{The Lower Cretaceous Petroleum System}

This petroleum system is generally associated with the rift Phase I and the basal part (Cenomanian) of rift Phase II in the WCARS basins (Figure 7). Petroleum accumulations occur in sandstones of Aptian to Cenomanian alluvial/braided/meandering rivers, and coastal marine and lacustrine delta deposits (Table 1). In the Muglad Basin of Sudan these sandy reservoirs constitute mediumcoarse grained sandstones of the upper Albian-Cenomanian Bentiu Formation with porosity 15\% - 27\% at depth interval of up to $3595 \mathrm{~m}$ [51]. In the Doba and Doseo Basins of the Chad Republic, the sandstones are fine to coarse grained, poorly-fairly sorted and in places conglomeratic. Porosity ranges from $12 \%$ to $24 \%$ (ave. $18 \%$ ) and permeability is $3-25 \mathrm{md}$ (ave. $15 \mathrm{md}$ ) at a depth range of $1500-2700 \mathrm{~m}$ [38]. In Termit Basin of Chad and Niger Republics, deltaic to tidal sandstones of Cenomanian Sedigi Formation constitute the reservoir.

The source rocks of this system are the Lower Cretaceous (pre-Aptian to Albian) lacustrine shales (Table 1) deposited mainly at the axial part of the rift system in a dysoxic to anoxic setting. They are generally rich in total organic carbon (TOC) and are composed of mainly type I (oil-generating) organic matter (OM). In the Muglad Basin, these source rocks constitute the Neocomian-Barremain Sharaf and Abu Gabra Formations rich in amorphous kerogen (>80\%) with TOC ranges of $1.5-2.3$ wt $\%$ and high values of hydrogen index (HI) of 338 - 546 mg HC/g TOC [52]. This suggests mainly type I OM. These source rocks (e.g. Tefidet, Alaniara and Tegama Formations) in Niger and Chad Republics basins contain TOC that ranges from 1 - $14 \mathrm{wt} \%$ with predominantly type I OM (HI > $600 \mathrm{mg} \mathrm{HC/g} \mathrm{TOC)} \mathrm{derived} \mathrm{from} \mathrm{fresh}$ water algae and bacteria [38].

Local seal rocks (3 - $5 \mathrm{~m}$ thick) exist as interbedded Lower Cretaceous lacustrine shales while regional seals are provided by the Upper Cretaceous fluvial and lacustrine shales in the Muglad Basin (e.g. Aradeiba and Zarga Formations) and predominantly marine shales in Niger and Chad basins.

1) The Northern Benue Trough

In the northeastern Nigerian sector of the Benue Trough, potential Lower Cretaceous Petroleum System includes sediments of the alluvial-braided-lacustrine $\mathrm{Bi}$ ma and the transitional (barrier island-lagoon and deltaic) Yolde Formations in both the Yola and the Gongola Sub-basins (Figures 5, 14).

The potential reservoirs are the alluvial fans, braided river channel and lacustrine deltaic sandstones of the Bima Formation, as well as, the barrier ridges and inlet channel sandstones and the flood and ebb deltas of the barrier island complex of the Yolde Formation. Sandstone thicknesses in the lower and upper Bima Formation are in the range of $3-10 \mathrm{~m}$ and may be more than $100 \mathrm{~m}$ where amalgamated. Sandstone thickness in the Yolde Formation ranges between $1-10 \mathrm{~m}$ [53]. Porosity and permeability data of these potential reservoirs are very scarce. In the Gongola Sub-basin, however, porosity varied from $5.58 \%-29.22 \%$ and permeability is in the range of 10.67 - $89.27 \mathrm{md}$ [54]. The sandstones of the 
Table 1. Petroleum discovery wells in Niger and Chad Republics showing stratigraphic levels and rock type of reservoirs and the associated petroleum system (data from Genik, 1993).

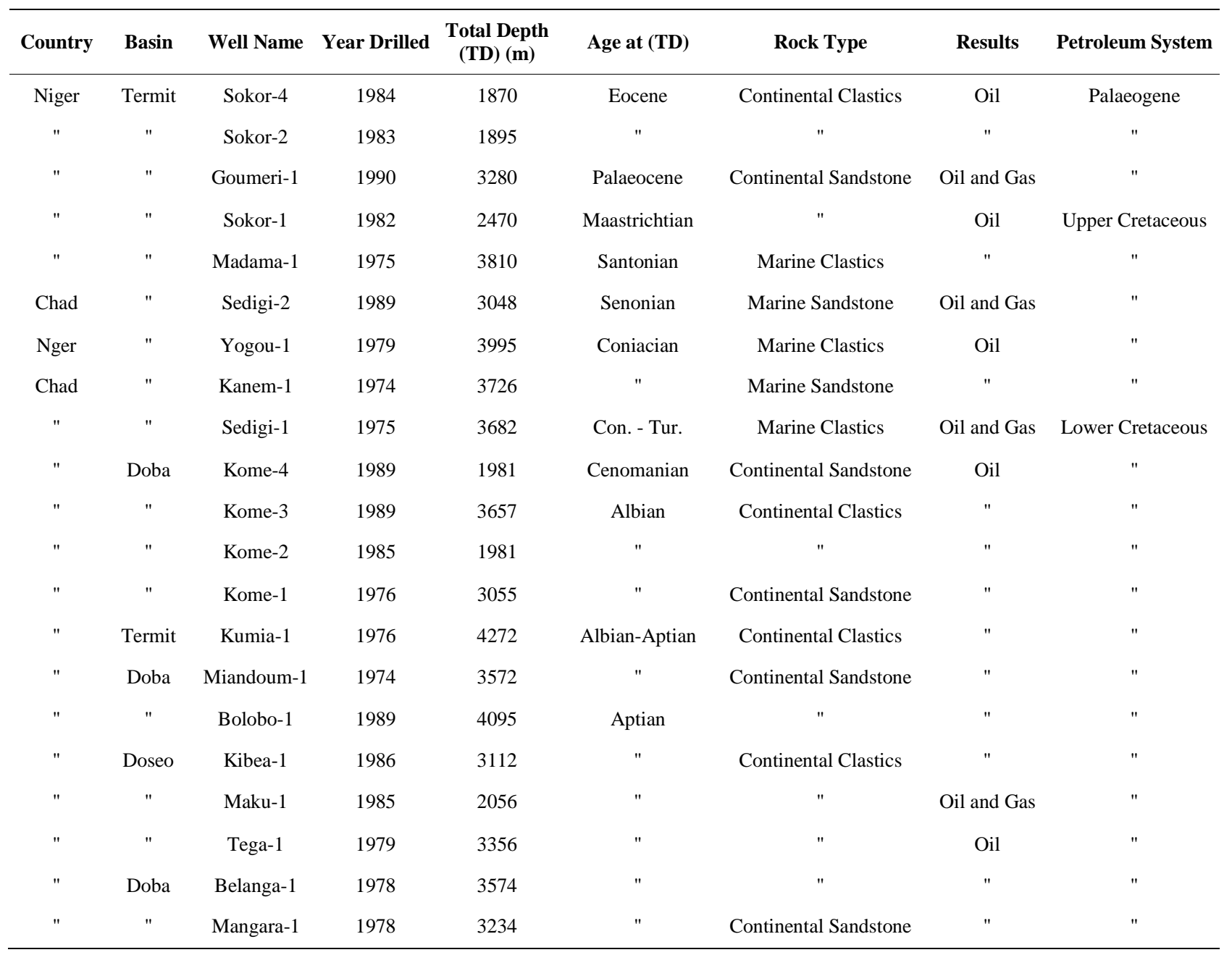
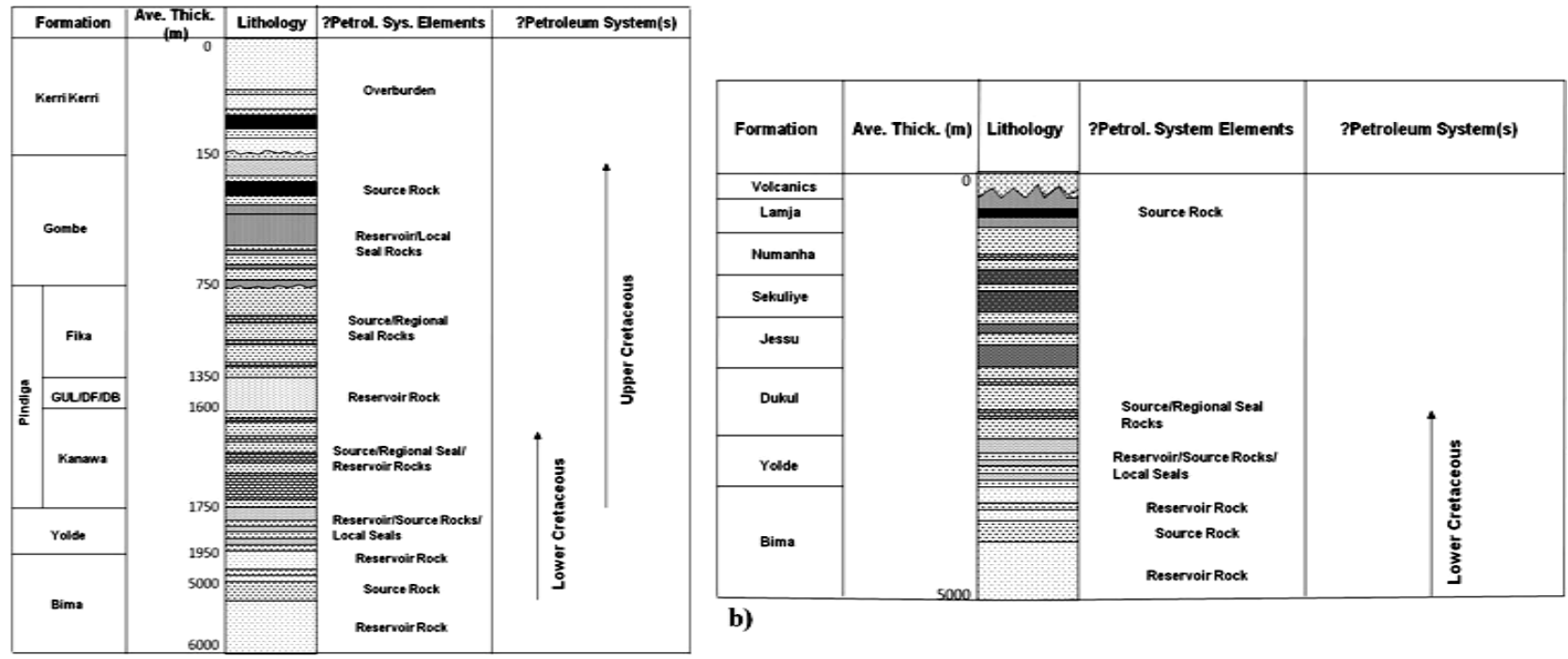

b)

a)

Figure 14. Potential petroleum system(s) in, (a) the Gongola Sub-basin and (b) The Yola Sub-basin, Northern Benue Trough. Note: GUL/DF/DB represents Gulani/Daban Fulani/Dumbulwa Members. 
Yolde Formation, on the other hand, are generally moderately well sorted and constitute very important aquifer in both the Yola and the Gongola Sub-basins.

Potential source rocks of this system are the interbedded shales of the Bima and Yolde Formations. These shales are fluvio-lacustrine lacustrine in the Bima Formation, and marine to lagoonal in the Yolde Formation. Although little is known on the distribution of the lacustrine facies in the Gongola Sub-basin, [15] and [34] reported the presence of some $350 \mathrm{~m}$ of alternating shales, silty shales, fine to coarse grained sandstones and minor carbonates in the core of Lamurde anticline. Reference [34] interpreted this succession as lacustrine-related shales and delta sandstones. Reference [55] interpreted it as part of a regional lacustrine and peri-lacustrine succession that existed at depth over much areas of the Northern Benue Trough. Reference [56], however, regarded the lacustrine facies as strictly local to the Lamurde area. Source rock assessment of the Bima Formation of the Gongola Sub-basin (Table 2) indicates TOC range of $0.10-0.87 \mathrm{wt} \%$ with an average of mere 0.32 wt\% (Table 3). Only $19.0 \%$ of the samples from the studied data (Table 2, excluding samples Nas 53, 54, 55 from the well Nasara-1) shows TOC values $\geq 0.5$ wt $\%$. HIs are equally low ranging from $21-160 \mathrm{mgHC} / \mathrm{gTOC}$ with an average of $73 \mathrm{mg} \mathrm{HC} / \mathrm{g}$ TOC (Table 3). This indicates the dominance of terrestrially derived type III OM capable of generating mainly gas. An exception to this interpretation, however, is the Rock Eval pyrolysis data of the samples Nas 53, 54 and 55, representing a depth interval of $60 \mathrm{ft}(\approx 18 \mathrm{~m})$ from $4710 \mathrm{ft}(\approx 1436 \mathrm{~m})$ $4770 \mathrm{ft}(\approx 1454 \mathrm{~m})$ in the well Nasara-1 drilled by Chevron in the Gongola Sub-basin. At this depth interval, the TOCs and HIs are anomalously high (52.10 - 55.20 wt\% and 564 - 589 mg HC/g TOC respectively, Table 2) with averages of $53 \mathrm{wt} \%$ and $574 \mathrm{mg}$ HC/g TOC respectively (Table 3), and the lithology is sandy [46]. This, coupled with the bimodality of the $S_{2}$ peak (pyrolysable hydrocarbon yield) of the Rock Eval pyrogram (Figure 15), high extract yield (Table 4) and predominance of oilrelated macerals (i.e. fluorinite and exsudatinite, Figure 16) suggest the presence of reservoired migrated oil at the depth interval (Figure 17). Very low extended hopane distribution of $\leq 0.27$ (H31R/H30 ratios, Table 5) indicates that the oil was generated from lacustrine sediments [46]. These sediments may be the lacustrine shales of the Bima Formation not yet penetrated by the well Nasara-1. Therefore, this may also attests to the presence of effective and mature type I (oil-generating) source rock of lacustrine origin at deeper stratigraphic levels in the Northern Benue Trough (source rock data from the Yola Sub-basin is not available but may mimic those of the Gongola Sub-basin). Potential source rocks from the Cenomanian Yolde Formation, on the other hand, show

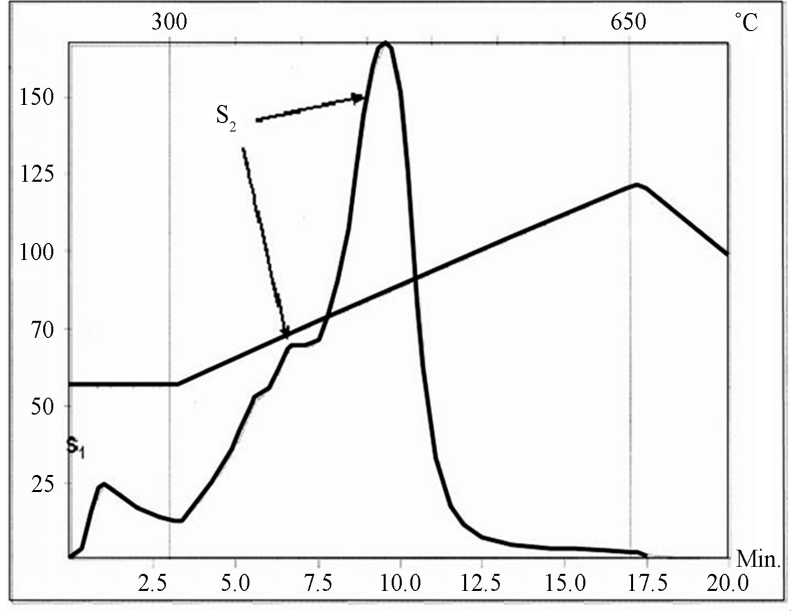

Figure 15. A pyrogram of sample NAS 53 showing bimodal $S_{2}$ peak.

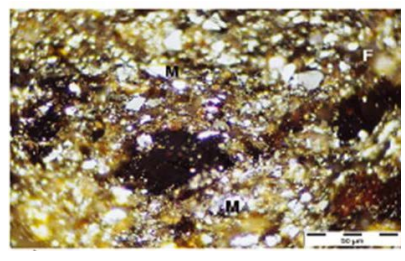

(a)

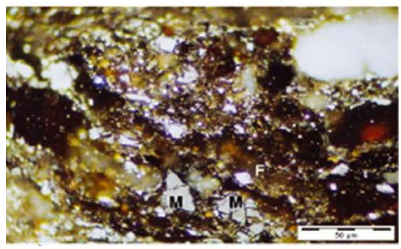

(c)

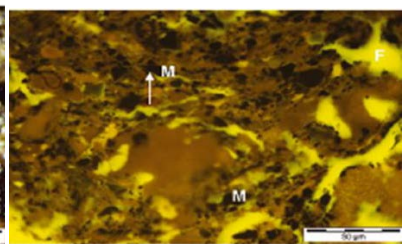

(b)

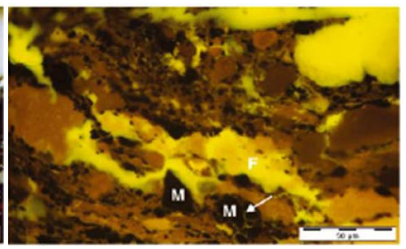

(d)
Figure 16. Maceral composition of sample NAS 53 under reflected white light (upper) and fluorescent light (lower). (F) Fluorinite perhaps associated with exsudatinite; (M) Mineral matter, mainly quartz and clays. Note the infilling (arrow) of the fractures of the mineral matter by fluorinite in (c) and (d).

TOC values of $0.30-0.35 \mathrm{wt} \%$ from the Gongola Subbasin with an average of $0.33 \%$ (Table 3) and 0.10 $12.90 \mathrm{wt} \%$ with an average of $2.00 \mathrm{wt} \%$ in the Yola Subbasin (Table 6). HIs range from 26 - $31 \mathrm{mg} \mathrm{HC/g}$ TOC in the Gongola Sub-basin suggesting type IV organic matter (Table 3). In the Yola Sub-basin, however, the HIs range from 27 - $171 \mathrm{mg} \mathrm{HC/g}$ TOC with an average of $60 \mathrm{mg}$ HC/g TOC (Table 6) suggesting the predominance of type III organic matter but with localized presence of oil and gas generating type II organic matter. Generally, the potential source rocks of the Lower Cretaceous Petroleum System in the Northern Benue Trough (both Yola and Gongola Sub-basins) are mature for hydrocarbon generation showing average $T_{\max }$ values that are generally above the minimum threshold of $435^{\circ} \mathrm{C}$ (Tables 3, 6). 
Table 2. Rock-Eval pyrolysis data of samples from the Northern Benue Trough [43,46,57-59].

\begin{tabular}{|c|c|c|c|c|c|c|c|c|c|c|c|c|c|c|}
\hline $\mathbf{S} / \mathbf{N}$ & $\begin{array}{l}\text { Sample } \\
\text { Name }\end{array}$ & $\begin{array}{l}\text { Sample } \\
\text { Loc. }\end{array}$ & $\begin{array}{c}\text { Basin/ } \\
\text { Sub-basin }\end{array}$ & $\begin{array}{l}\text { Form./ } \\
\text { Lithol. }\end{array}$ & Age & TOC & S1 & S2 & S3 & Tmax & HI & OI & PI & Source \\
\hline 1 & KM3 & $\begin{array}{l}\text { K/Borehole } \\
\text { (GSN4041) }\end{array}$ & $\begin{array}{c}\text { NBT/ } \\
\text { Gongola }\end{array}$ & $\begin{array}{l}\text { Gombe/ } \\
\text { Shale }\end{array}$ & $\begin{array}{l}\text { Campan.- } \\
\text { Maastr. }\end{array}$ & 1.46 & 0.03 & 0.96 & & 433.00 & 65.00 & & 0.97 & [58] \\
\hline 2 & KM4 & $"$ & $"$ & $"$ & $"$ & 1.20 & 0.01 & 0.27 & & 422.00 & 22.00 & & 0.96 & $"$ \\
\hline 3 & GMC1 & H/Gari & $"$ & $"$ & $"$ & 0.55 & 0.02 & 0.10 & & 418.00 & 18.00 & & 0.83 & [57] \\
\hline 4 & GMC7 & $"$ & $"$ & $"$ & $"$ & 0.25 & 0.01 & 0.10 & & 418.00 & 0.00 & & 0.91 & $"$ \\
\hline 5 & GMC14 & $"$ & $"$ & $"$ & $"$ & 0.20 & 0.01 & 0.00 & & 418.00 & 0.00 & & 0.00 & $"$ \\
\hline 6 & UBHJ1 & $"$ & $"$ & $"$ & $"$ & 0.92 & 0.01 & 0.03 & 0.47 & 282.00 & 3.00 & 51.00 & 0.75 & [43] \\
\hline 7 & UBHJ2 & $"$ & $"$ & $"$ & $"$ & 0.83 & 0.01 & 0.03 & 0.47 & 300.00 & 4.00 & 57.00 & 0.75 & $"$ \\
\hline 8 & UBHJ3 & $"$ & $"$ & $"$ & $"$ & 0.96 & 0.01 & 0.03 & 0.43 & 502.00 & 3.00 & 45.00 & 0.75 & $"$ \\
\hline 9 & UBHJ4 & $"$ & $"$ & $\begin{array}{l}\text { Gombe/ } \\
\text { Coaly Shale }\end{array}$ & $"$ & 1.05 & 0.01 & 0.03 & 0.37 & 310.00 & 3.00 & 35.00 & 0.75 & {$[46]$} \\
\hline 10 & UBWJ1 & W/Sale & $"$ & $"$ & $"$ & 1.26 & 0.01 & 0.05 & 0.67 & 515.00 & 4.00 & 53.00 & 0.83 & [43] \\
\hline 11 & UBWJ2 & $"$ & $"$ & $"$ & $"$ & 2.63 & 0.01 & 0.06 & 2.60 & 511.00 & 2.00 & 99.00 & 0.86 & $"$ \\
\hline 12 & UBDJ1 & D/Borehole & $"$ & $"$ & $"$ & 6.84 & 0.13 & 12.01 & 5.08 & 429.00 & 176.00 & 74.00 & 0.99 & [46] \\
\hline 13 & MGMC3 & $"$ & $"$ & $"$ & $"$ & 3.43 & 0.08 & 9.62 & 1.58 & 432.00 & 280.00 & 46.00 & 0.99 & $"$ \\
\hline 14 & UBDJ2 & $"$ & $"$ & $\begin{array}{l}\text { Gombe/ } \\
\text { Shaly Coal }\end{array}$ & $"$ & 20.20 & 0.62 & 35.95 & 10.53 & 423.00 & 178.00 & 52.00 & 0.98 & $"$ \\
\hline 15 & CP8 & $\begin{array}{l}\text { Maiganga/ } \\
\text { Borehole }\end{array}$ & $"$ & $"$ & $"$ & 14.90 & 0.79 & 18.19 & 11.30 & 435.00 & 122.08 & 75.84 & 0.96 & $"$ \\
\hline 16 & СР13 & $"$ & $"$ & $"$ & $"$ & 23.70 & 0.80 & 32.60 & 14.77 & 423.00 & 137.55 & 62.32 & 0.98 & $"$ \\
\hline 17 & Lamco1 & Lamja & $\begin{array}{l}\text { NBT/ } \\
\text { Yola }\end{array}$ & $\begin{array}{l}\text { Lamja/ } \\
\text { Coal }\end{array}$ & $\begin{array}{l}\text { U. Cenom. - } \\
\text { Sant. }\end{array}$ & 50.70 & 2.15 & 93.25 & 12.62 & 438.00 & 184.00 & 25.00 & 0.98 & [43] \\
\hline 18 & Lamco2 & $"$ & $"$ & $"$ & $"$ & 51.10 & 1.47 & 91.70 & 14.15 & 438.00 & 179.00 & 28.00 & 0.98 & $"$ \\
\hline 19 & LK5 & Lakun & $"$ & $\begin{array}{l}\text { Jessu/ } \\
\text { Shale }\end{array}$ & $"$ & 0.37 & 0.01 & 0.05 & & 433.00 & 31.00 & & 0.83 & [59] \\
\hline 20 & LK6 & $"$ & $"$ & $"$ & $"$ & 0.21 & 0.00 & 0.05 & & 434.00 & 11.00 & & 1.00 & $"$ \\
\hline 21 & LK7 & $"$ & $"$ & $"$ & $"$ & 0.32 & 0.01 & 0.07 & & 431.00 & 18.00 & & 0.88 & $"$ \\
\hline 22 & LK8 & $"$ & $"$ & $"$ & $"$ & 0.43 & 0.01 & 0.07 & & 431.00 & 16.00 & & 0.88 & $"$ \\
\hline 23 & LK9 & $"$ & $"$ & $"$ & $"$ & 0.51 & 0.03 & 0.52 & & 436.00 & 35.00 & & 0.95 & $"$ \\
\hline 24 & KT5 & Kutare & $"$ & $"$ & $"$ & 0.71 & 0.02 & 0.10 & & 432.00 & 13.00 & & 0.83 & $"$ \\
\hline 25 & KT6 & $"$ & $"$ & $"$ & $"$ & 0.75 & 0.02 & 0.18 & & 435.00 & 28.00 & & 0.90 & $"$ \\
\hline 26 & KT7 & $"$ & $"$ & $"$ & $"$ & 0.85 & 0.02 & 0.28 & & 432.00 & 49.00 & & 0.93 & $"$ \\
\hline 27 & FKS5 & Nafada & $\begin{array}{l}\text { NBT/ } \\
\text { Gongola }\end{array}$ & $\begin{array}{l}\text { U. Pindiga/ } \\
\text { Shale }\end{array}$ & $"$ & 0.05 & 0.03 & 0.09 & & 591.00 & 180.00 & & 0.75 & [57] \\
\hline 28 & FKS9 & $"$ & $"$ & $"$ & $"$ & 0.05 & 0.04 & 0.09 & & 586.00 & 180.00 & & 0.69 & $"$ \\
\hline 29 & FKS11 & $"$ & $"$ & $"$ & " & 0.04 & 0.03 & 0.06 & & 584.00 & 150.00 & & 0.67 & $"$ \\
\hline 30 & FKS14 & $"$ & $"$ & $"$ & $"$ & 0.39 & 0.02 & 0.02 & & 445.00 & 5.00 & & 0.50 & $"$ \\
\hline 31 & KM9 & $\begin{array}{l}\text { K/Borehole } \\
\text { (GSN4041) }\end{array}$ & " & $\begin{array}{l}\text { Pindiga/ } \\
\text { Shale }\end{array}$ & $"$ & 2.45 & 0.02 & 1.88 & & 435.00 & 76.00 & & 0.99 & [58] \\
\hline
\end{tabular}




\section{Continued}

\begin{tabular}{|c|c|c|c|c|c|c|c|c|c|c|c|c|c|c|}
\hline 32 & KM11 & $"$ & $"$ & $"$ & " & 1.63 & 0.01 & 0.22 & & 415.00 & 13.00 & & 0.96 & $"$ \\
\hline 33 & KM13 & $"$ & $"$ & $"$ & " & 1.56 & 0.01 & 0.31 & & 416.00 & 19.00 & & 0.97 & $"$ \\
\hline 34 & KM16 & $"$ & $"$ & $"$ & $"$ & 0.60 & 0.00 & 0.09 & & 423.00 & 15.00 & & 1.00 & $"$ \\
\hline 35 & KM17 & $"$ & $"$ & $"$ & " & 0.74 & 0.02 & 0.10 & & 422.00 & 13.00 & & 0.83 & $"$ \\
\hline 36 & KM18 & $"$ & $"$ & $"$ & " & 0.39 & 0.00 & 0.04 & & 426.00 & 10.00 & & 1.00 & $"$ \\
\hline 37 & KM19 & $"$ & $"$ & $"$ & " & 0.28 & 0.01 & 0.04 & & 0.00 & 14.00 & & 0.80 & $"$ \\
\hline 38 & KM21 & $"$ & $"$ & $"$ & $"$ & 0.65 & 0.00 & 0.09 & & 425.00 & 13.00 & & 1.00 & $"$ \\
\hline 39 & KM23 & $"$ & $"$ & $"$ & " & 0.54 & 0.01 & 0.05 & & 419.00 & 9.00 & & 0.83 & $"$ \\
\hline 40 & KM25 & $"$ & $"$ & $"$ & " & 0.21 & 0.01 & 0.11 & & 0.00 & 0.00 & & 0.92 & " \\
\hline 41 & GB1 & $\begin{array}{l}\text { G/Borehole } \\
\text { (GSN1504) }\end{array}$ & $"$ & $"$ & $"$ & 0.57 & 0.01 & 0.09 & & 426.00 & 15.00 & & 0.90 & $"$ \\
\hline 42 & GB3 & $"$ & $"$ & $"$ & " & 0.60 & 0.02 & 0.10 & & 423.00 & 24.00 & & 0.83 & $"$ \\
\hline 43 & GB6 & $"$ & $"$ & $"$ & $"$ & 0.35 & 0.00 & 0.08 & & 428.00 & 22.00 & & 1.00 & $"$ \\
\hline 44 & GB8 & $"$ & $"$ & $"$ & " & 0.46 & 0.03 & 0.08 & & 421.00 & 17.00 & & 0.73 & $"$ \\
\hline 45 & GB10 & $"$ & $"$ & $"$ & $"$ & 0.47 & 0.01 & 0.08 & & 422.00 & 23.00 & & 0.89 & $"$ \\
\hline 46 & GB13 & $"$ & $"$ & $"$ & " & 0.49 & 0.02 & 0.18 & & 424.00 & 36.00 & & 0.90 & $"$ \\
\hline 47 & GB14 & $"$ & $"$ & $"$ & " & 0.45 & 0.01 & 0.07 & & 419.00 & 16.00 & & 0.88 & $"$ \\
\hline 48 & GB16 & $"$ & $"$ & $"$ & " & 0.32 & 0.01 & 0.07 & & 425.00 & 21.00 & & 0.88 & $"$ \\
\hline 49 & GB17 & $"$ & $"$ & $"$ & " & 0.48 & 0.01 & 0.14 & & 419.00 & 29.00 & & 0.93 & $"$ \\
\hline 50 & GB19 & $"$ & $"$ & $"$ & " & 0.43 & 0.01 & 0.07 & & 419.00 & 16.00 & & 0.88 & $"$ \\
\hline 51 & GB21 & $"$ & $"$ & $"$ & " & 0.42 & 0.02 & 0.08 & & 425.00 & 19.00 & & 0.80 & $"$ \\
\hline 52 & GB22 & $"$ & $"$ & $"$ & $"$ & 0.40 & 0.00 & 0.15 & & 420.00 & 37.00 & & 1.00 & $"$ \\
\hline 53 & GB26 & $"$ & $"$ & $"$ & $"$ & 0.38 & 0.01 & 0.15 & & 423.00 & 39.00 & & 0.94 & $"$ \\
\hline 54 & GB28 & $"$ & $"$ & $"$ & $"$ & 0.46 & 0.02 & 0.14 & & 424.00 & 30.00 & & 0.88 & $"$ \\
\hline 55 & GB31 & $"$ & $"$ & $"$ & $"$ & 0.40 & 0.01 & 0.11 & & 424.00 & 27.00 & & 0.92 & $"$ \\
\hline 56 & AS1 & Ashaka Quarry & $"$ & $"$ & $"$ & 0.41 & 0.00 & 0.10 & & 423.00 & 24.00 & & 1.00 & $"$ \\
\hline 57 & AS2 & $"$ & $"$ & $"$ & $"$ & 0.26 & 0.00 & 0.04 & & 431.00 & 15.00 & & 1.00 & $"$ \\
\hline 58 & DA7 & Pindiga & $"$ & $"$ & $"$ & 2.13 & 0.07 & 0.73 & & 424.00 & 34.00 & & 0.91 & [57] \\
\hline 59 & DA11 & $"$ & $"$ & $"$ & $"$ & 2.08 & 0.07 & 0.63 & & 423.00 & 32.00 & & 0.90 & $"$ \\
\hline 60 & DA12 & $"$ & $"$ & $"$ & " & 1.94 & 0.05 & 0.32 & & 419.00 & 16.00 & & 0.86 & $"$ \\
\hline 61 & GGS3 & Ashaka Quarry & $"$ & $"$ & " & 0.52 & 0.01 & 0.09 & & 418.00 & 17.00 & & 0.90 & $"$ \\
\hline 62 & GGS12 & $"$ & $"$ & $"$ & $"$ & 0.50 & 0.02 & 0.10 & & 419.00 & 20.00 & & 0.83 & $"$ \\
\hline 63 & GGS13 & $"$ & $"$ & $"$ & $"$ & 0.51 & 0.02 & 0.07 & & 418.00 & 14.00 & & 0.78 & $"$ \\
\hline 64 & GGL16 & $"$ & $"$ & $"$ & $"$ & 0.10 & 0.01 & 0.02 & & 483.00 & 20.00 & & 0.67 & $"$ \\
\hline 65 & GGS17 & $"$ & $"$ & $"$ & $"$ & 0.57 & 0.04 & 0.19 & & 417.00 & 33.00 & & 0.83 & $"$ \\
\hline 66 & GGS21 & $"$ & $"$ & $"$ & $"$ & 0.46 & 0.02 & 0.05 & & 416.00 & 11.00 & & 0.71 & $"$ \\
\hline 67 & PIND10 & Pindiga & $"$ & $"$ & $"$ & 0.71 & 0.02 & 0.22 & 0.36 & 418.00 & 31.00 & 51.00 & 0.92 & [43] \\
\hline
\end{tabular}




\section{Continued}

\begin{tabular}{|c|c|c|c|c|c|c|c|c|c|c|c|c|c|c|}
\hline 68 & GONG1 & Ashaka Quarry & $"$ & $"$ & $"$ & 0.59 & 0.02 & 0.12 & 0.35 & 419.00 & 20.00 & 60.00 & 0.86 & $"$ \\
\hline 69 & GONG2 & $"$ & $"$ & $"$ & $"$ & 0.52 & 0.01 & 0.09 & 0.26 & 420.00 & 17.00 & 50.00 & 0.90 & $"$ \\
\hline 70 & GONG3 & $"$ & $"$ & $"$ & $"$ & 0.53 & 0.01 & 0.08 & 0.32 & 417.00 & 15.00 & 61.00 & 0.89 & $"$ \\
\hline 71 & GONG4 & $"$ & $"$ & $"$ & $"$ & 0.55 & 0.02 & 0.14 & 0.33 & 421.00 & 26.00 & 61.00 & 0.88 & $"$ \\
\hline 72 & Nas-1 & Well Nasara-1 & $"$ & $"$ & $"$ & 0.67 & 0.01 & 0.12 & 0.40 & 419.00 & 18.00 & 60.00 & 0.92 & [46] \\
\hline 73 & Nas-2 & $"$ & $"$ & $"$ & $"$ & 0.87 & 0.02 & 0.29 & 0.67 & 420.00 & 33.00 & 77.00 & 0.94 & $"$ \\
\hline 74 & Nas-3 & $"$ & $"$ & $"$ & $"$ & 0.65 & 0.01 & 0.20 & 0.31 & 420.00 & 31.00 & 48.00 & 0.95 & $"$ \\
\hline 75 & Nas-4 & $"$ & $"$ & $"$ & $"$ & 0.63 & 0.01 & 0.14 & 0.41 & 420.00 & 22.00 & 65.00 & 0.93 & $"$ \\
\hline 76 & Nas-5 & $"$ & $"$ & " & $"$ & 0.55 & 0.01 & 0.08 & 0.34 & 421.00 & 15.00 & 62.00 & 0.89 & $"$ \\
\hline 77 & Nas-6 & $"$ & $"$ & $"$ & $"$ & 0.51 & 0.01 & 0.08 & 0.34 & 423.00 & 16.00 & 67.00 & 0.89 & $"$ \\
\hline 78 & Nas-7 & $"$ & " & $"$ & $"$ & 0.64 & 0.01 & 0.16 & 0.36 & 421.00 & 25.00 & 57.00 & 0.94 & $"$ \\
\hline 79 & Nas-8 & $"$ & $"$ & $"$ & $"$ & 0.58 & 0.01 & 0.11 & 0.34 & 423.00 & 19.00 & 59.00 & 0.92 & $"$ \\
\hline 80 & Nas-9 & " & " & " & $"$ & 0.66 & 0.01 & 0.10 & 0.25 & 424.00 & 15.00 & 38.00 & 0.91 & $"$ \\
\hline 81 & Nas-10 & $"$ & " & " & " & 0.58 & 0.01 & 0.11 & 0.38 & 424.00 & 19.00 & 65.00 & 0.92 & $"$ \\
\hline 82 & Nas-11 & $"$ & $"$ & " & $"$ & 0.46 & 0.01 & 0.10 & 0.36 & 427.00 & 22.00 & 78.00 & 0.91 & $"$ \\
\hline 83 & Nas-12 & $"$ & " & " & " & 0.45 & 0.00 & 0.08 & 0.37 & 426.00 & 18.00 & 82.00 & 1.00 & $"$ \\
\hline 84 & Nas-13 & $"$ & $"$ & $"$ & " & 0.51 & 0.01 & 0.10 & 0.34 & 424.00 & 20.00 & 67.00 & 0.91 & $"$ \\
\hline 85 & Nas-14 & $"$ & $"$ & $"$ & $"$ & 0.56 & 0.01 & 0.13 & 0.57 & 420.00 & 23.00 & 102.00 & 0.93 & $"$ \\
\hline 86 & Nas-15 & $"$ & $"$ & $"$ & " & 0.57 & 0.01 & 0.14 & 0.44 & 421.00 & 24.00 & 77.00 & 0.93 & $"$ \\
\hline 87 & Nas-16 & $"$ & " & " & $"$ & 0.55 & 0.01 & 0.15 & 0.56 & 424.00 & 27.00 & 102.00 & 0.94 & $"$ \\
\hline 88 & Nas-17 & $"$ & $"$ & $"$ & $"$ & 0.59 & 0.01 & 0.15 & 0.39 & 420.00 & 26.00 & 66.00 & 0.94 & $"$ \\
\hline 89 & Nas-18 & $"$ & " & " & $"$ & 0.53 & 0.01 & 0.13 & 0.57 & 424.00 & 25.00 & 108.00 & 0.93 & $"$ \\
\hline 90 & Nas-19 & $"$ & " & " & $"$ & 0.48 & 0.01 & 0.14 & 0.47 & 423.00 & 29.00 & 98.00 & 0.93 & $"$ \\
\hline 91 & Nas-20 & $"$ & $"$ & $"$ & $"$ & 0.50 & 0.01 & 0.14 & 0.47 & 423.00 & 28.00 & 93.00 & 0.93 & $"$ \\
\hline 92 & Nas-21 & $"$ & $"$ & $"$ & " & 0.44 & 0.01 & 0.10 & 0.35 & 424.00 & 23.00 & 79.00 & 0.91 & $"$ \\
\hline 93 & Nas-22 & $"$ & $"$ & $"$ & " & 0.44 & 0.01 & 0.11 & 0.46 & 424.00 & 25.00 & 104.00 & 0.92 & $"$ \\
\hline 94 & Nas-23 & $"$ & " & " & $"$ & 0.45 & 0.01 & 0.09 & 0.31 & 425.00 & 20.00 & 69.00 & 0.90 & $"$ \\
\hline 95 & Nas-24 & $"$ & $"$ & $"$ & " & 0.40 & 0.01 & 0.09 & 0.37 & 426.00 & 22.00 & 92.00 & 0.90 & $"$ \\
\hline 96 & Nas-25 & $"$ & $"$ & $"$ & $"$ & 0.54 & 0.01 & 0.14 & 0.55 & 422.00 & 26.00 & 102.00 & 0.93 & $"$ \\
\hline 97 & Nas-26 & $"$ & $"$ & $"$ & " & 0.59 & 0.01 & 0.14 & 0.31 & 419.00 & 24.00 & 53.00 & 0.93 & $"$ \\
\hline 98 & Nas-27 & $"$ & $"$ & " & $"$ & 0.59 & 0.01 & 0.17 & 0.51 & 420.00 & 29.00 & 86.00 & 0.94 & $"$ \\
\hline 99 & Nas-28 & $"$ & $"$ & $"$ & $"$ & 0.46 & 0.01 & 0.16 & 0.51 & 426.00 & 35.00 & 110.00 & 0.94 & $"$ \\
\hline 100 & Nas-29 & " & " & " & $"$ & 0.50 & 0.02 & 0.26 & 0.48 & 429.00 & 52.00 & 96.00 & 0.93 & $"$ \\
\hline 101 & Nas-30 & $"$ & $"$ & $"$ & " & 0.53 & 0.02 & 0.30 & 0.55 & 427.00 & 57.00 & 104.00 & 0.94 & $"$ \\
\hline 102 & Nas-31 & $"$ & " & $"$ & $"$ & 0.75 & 0.03 & 0.58 & 0.40 & 430.00 & 77.00 & 53.00 & 0.95 & $"$ \\
\hline 103 & Nas-32 & $"$ & " & $"$ & $"$ & 0.71 & 0.03 & 0.48 & 0.53 & 433.00 & 68.00 & 75.00 & 0.94 & $"$ \\
\hline
\end{tabular}




\section{Continued}

\begin{tabular}{|c|c|c|c|c|c|c|c|c|c|c|c|c|c|c|}
\hline 104 & Nas-33 & $"$ & $"$ & $"$ & $"$ & 0.58 & 0.03 & 0.33 & 0.63 & 423.00 & 57.00 & 108.00 & 0.92 & $"$ \\
\hline 105 & Nas-34 & $"$ & $"$ & $"$ & $"$ & 0.59 & 0.01 & 0.29 & 0.45 & 433.00 & 50.00 & 77.00 & 0.97 & $"$ \\
\hline 106 & MPS20 & $\begin{array}{l}\text { Pindiga Water } \\
\text { borehole }\end{array}$ & $"$ & $"$ & $"$ & 0.30 & 0.06 & 0.08 & 0.31 & 421.00 & 26.00 & 102.00 & 0.57 & $"$ \\
\hline 107 & MPS50 & $"$ & $"$ & $"$ & $"$ & 0.57 & 0.02 & 0.20 & 0.34 & 421.00 & 35.00 & 60.00 & 0.91 & $"$ \\
\hline 108 & MPS63 & $"$ & $"$ & $"$ & $"$ & 0.52 & 0.02 & 0.20 & 0.27 & 417.00 & 38.00 & 52.00 & 0.91 & $"$ \\
\hline 109 & MPS70 & $"$ & $"$ & $"$ & $"$ & 0.47 & 0.01 & 0.15 & 0.28 & 419.00 & 32.00 & 59.00 & 0.94 & $"$ \\
\hline 110 & MPS72 & Gombe & $"$ & $"$ & $"$ & 0.64 & 0.02 & 0.21 & 0.33 & 421.00 & 33.00 & 52.00 & 0.91 & $"$ \\
\hline 111 & MPS77 & Balbaya & $"$ & $"$ & $"$ & 0.23 & 0.01 & 0.02 & 0.32 & 476.00 & 9.00 & 139.00 & 0.67 & $"$ \\
\hline 112 & MGS5 & Ashaka Quarry & $"$ & $"$ & $"$ & 0.42 & 0.01 & 0.06 & 0.34 & 421.00 & 14.00 & 81.00 & 0.86 & $"$ \\
\hline 113 & MGS2 & $"$ & $"$ & $"$ & $"$ & 0.50 & 0.01 & 0.11 & 0.22 & 423.00 & 22.00 & 44.00 & 0.92 & $"$ \\
\hline 114 & MGS1 & $"$ & $"$ & $\begin{array}{l}\text { Pindiga/ } \\
\text { Calc. Shale }\end{array}$ & $"$ & 0.37 & 0.01 & 0.15 & 0.64 & 425.00 & 40.00 & 171.00 & 0.94 & $"$ \\
\hline 115 & NA1 & $\begin{array}{l}\text { N. Borehole } \\
\text { (GSN1612) }\end{array}$ & $\begin{array}{l}\text { NBT/ } \\
\text { Yola }\end{array}$ & $\begin{array}{l}\text { Dukul/ } \\
\text { Shale }\end{array}$ & $"$ & 0.25 & 0.00 & 0.06 & & 0.00 & 24.00 & & 1.00 & $"$ \\
\hline 116 & NA4 & $"$ & $"$ & $"$ & $"$ & 0.53 & 0.33 & 0.22 & & 431.00 & 41.00 & & 0.40 & $"$ \\
\hline 117 & NA6 & $"$ & $"$ & $"$ & $"$ & 0.33 & 0.00 & 0.08 & & 442.00 & 24.00 & & 1.00 & $"$ \\
\hline 118 & NA8 & $"$ & $"$ & $"$ & $"$ & 0.58 & 0.08 & 0.11 & & 442.00 & 18.00 & & 0.58 & $"$ \\
\hline 119 & DKS5 & $\begin{array}{c}\text { Dukul/ } \\
\text { Jessu }\end{array}$ & $"$ & $"$ & $"$ & 0.57 & 0.03 & 0.20 & & 436.00 & 35.00 & & 0.87 & [57] \\
\hline 120 & LK1 & Lakum & $"$ & $"$ & $"$ & 0.64 & 0.02 & 0.10 & & 438.00 & 20.00 & & 0.83 & [59] \\
\hline 121 & LK2 & $"$ & $"$ & $"$ & $"$ & 1.15 & 0.03 & 0.96 & & 434.00 & 64.00 & & 0.97 & $"$ \\
\hline 122 & LK3 & $"$ & $"$ & $"$ & $"$ & 0.48 & 0.01 & 0.08 & & 439.00 & 27.00 & & 0.89 & $"$ \\
\hline 123 & LK4 & $"$ & $"$ & $"$ & $"$ & 0.77 & 0.01 & 0.14 & & 431.00 & 55.00 & & 0.93 & $"$ \\
\hline 124 & KT1 & Kutare & $"$ & $"$ & $"$ & 0.41 & 0.02 & 0.12 & & 440.00 & 17.00 & & 0.86 & $"$ \\
\hline 125 & KT2 & $"$ & $"$ & $"$ & $"$ & 0.58 & 0.02 & 0.16 & & 435.00 & 23.00 & & 0.89 & $"$ \\
\hline 126 & KT3 & $"$ & $"$ & $"$ & $"$ & 0.55 & 0.03 & 0.08 & & 438.00 & 51.00 & & 0.73 & $"$ \\
\hline 127 & KT4 & $"$ & $"$ & $"$ & $"$ & 0.71 & 0.01 & 0.15 & & 435.00 & 39.00 & & 0.94 & $"$ \\
\hline 128 & MDS4 & Lakum & $"$ & $"$ & $"$ & 0.45 & 0.01 & 0.10 & 0.36 & 435.00 & 22.00 & 81.00 & 0.91 & [43] \\
\hline 129 & MDS11 & $"$ & $"$ & $"$ & $"$ & 0.91 & 0.02 & 0.26 & 0.60 & 432.00 & 28.00 & 66.00 & 0.93 & $"$ \\
\hline 130 & MDS13 & $"$ & $"$ & $"$ & $"$ & 0.53 & 0.01 & 0.09 & 0.39 & 434.00 & 17.00 & 74.00 & 0.90 & $"$ \\
\hline 131 & DUKL1 & $"$ & $"$ & $"$ & $"$ & 0.72 & 0.03 & 0.46 & 0.20 & 433.00 & 64.00 & 28.00 & 0.94 & $"$ \\
\hline 132 & DUKL3 & $"$ & $"$ & $"$ & $"$ & 0.36 & 0.01 & 0.10 & 0.17 & 436.00 & 28.00 & 47.00 & 0.91 & $"$ \\
\hline 133 & DUKL5 & $"$ & $"$ & $"$ & $"$ & 0.34 & 0.01 & 0.05 & 0.83 & 429.00 & 15.00 & 242.00 & 0.83 & $"$ \\
\hline 134 & DUKL8 & $"$ & $"$ & $"$ & $"$ & 0.61 & 0.02 & 0.27 & 0.18 & 429.00 & 45.00 & 30.00 & 0.93 & $"$ \\
\hline 135 & NA10 & $\begin{array}{l}\text { N. Borehole } \\
\text { (GSN1612) }\end{array}$ & $"$ & $\begin{array}{l}\text { Yolde/ } \\
\text { Shale }\end{array}$ & $\begin{array}{l}\text { Pre - to M. } \\
\text { Cenom. }\end{array}$ & 0.56 & 0.03 & 0.24 & & 442.00 & 48.00 & & 0.89 & [58] \\
\hline 136 & NA12 & $"$ & $"$ & $"$ & $"$ & 12.90 & 4.48 & 22.00 & & 438.00 & 171.00 & & 0.83 & $"$ \\
\hline 137 & NA17 & $"$ & $"$ & $"$ & $"$ & 0.33 & 0.01 & 0.09 & & 437.00 & 27.00 & & 0.90 & $"$ \\
\hline 138 & NA22 & $"$ & $"$ & $"$ & $"$ & 0.89 & 0.06 & 0.49 & & 437.00 & 55.00 & & 0.89 & $"$ \\
\hline
\end{tabular}




\section{Continued}

\begin{tabular}{|c|c|c|c|c|c|c|c|c|c|c|c|c|c|c|}
\hline 139 & NA23 & $"$ & $"$ & $"$ & $"$ & 0.58 & 0.02 & 0.28 & & 438.00 & 48.00 & & 0.93 & $"$ \\
\hline 140 & NA25 & $"$ & $"$ & $"$ & $"$ & 0.39 & 0.01 & 0.20 & & 442.00 & 55.00 & & 0.95 & $"$ \\
\hline 141 & NA27 & $"$ & $"$ & $"$ & $"$ & 0.10 & 0.00 & 0.11 & & 0.00 & 30.00 & & 1.00 & $"$ \\
\hline 142 & NA29 & $"$ & $"$ & $"$ & $"$ & 0.21 & 0.02 & 0.09 & & 0.00 & 42.00 & & 0.82 & $"$ \\
\hline 143 & YOLD2 & Futuk & $\begin{array}{c}\text { NBT/ } \\
\text { Gongola }\end{array}$ & $"$ & $"$ & 0.35 & 0.01 & 0.11 & 0.12 & 438.00 & 31.00 & 34.00 & 0.92 & [43] \\
\hline 144 & YOLD4 & $"$ & $"$ & $"$ & $"$ & 0.30 & 0.01 & 0.08 & 0.19 & 437.00 & 26.00 & 63.00 & 0.89 & $"$ \\
\hline 145 & Nas-35 & Well Nasara-1 & $"$ & Bima/Shale & $"$ & 0.59 & 0.02 & 0.31 & 0.52 & 427.00 & 52.00 & 88.00 & 0.94 & $"$ \\
\hline 146 & Nas-36 & $"$ & $"$ & $"$ & $"$ & 0.69 & 0.02 & 0.24 & 0.52 & 428.00 & 35.00 & 75.00 & 0.92 & $"$ \\
\hline 147 & Nas-37 & $"$ & $"$ & $"$ & $"$ & 0.87 & 0.05 & 1.23 & 0.44 & 437.00 & 142.00 & 51.00 & 0.96 & $"$ \\
\hline 148 & Nas-38 & $"$ & $"$ & $"$ & $"$ & 0.55 & 0.02 & 0.70 & 0.52 & 442.00 & 128.00 & 95.00 & 0.97 & $"$ \\
\hline 149 & MYS3 & Gombe & $"$ & $"$ & $"$ & 0.21 & 0.01 & 0.13 & 0.51 & 424.00 & 62.00 & 242.00 & 0.93 & $"$ \\
\hline 150 & Nas-39 & Well Nasara-1 & $"$ & $"$ & $"$ & 0.24 & 0.01 & 0.12 & 0.48 & 445.00 & 50.00 & 201.00 & 0.92 & $"$ \\
\hline 151 & Nas-40 & $"$ & $"$ & $"$ & $"$ & 0.25 & 0.00 & 0.13 & 0.39 & 445.00 & 52.00 & 156.00 & 1.00 & $"$ \\
\hline 152 & Nas-42 & $"$ & $"$ & $"$ & $"$ & 0.38 & 0.07 & 0.61 & 0.76 & 414.00 & 160.00 & 199.00 & 0.90 & $"$ \\
\hline 153 & Nas-43 & $"$ & $"$ & $"$ & $"$ & 0.49 & 0.02 & 0.21 & 0.41 & 463.00 & 43.00 & 84.00 & 0.91 & $"$ \\
\hline 154 & Nas-44 & $"$ & $"$ & $"$ & $"$ & 0.17 & 0.01 & 0.11 & 0.45 & 441.00 & 63.00 & 259.00 & 0.92 & $"$ \\
\hline 155 & Nas-45 & $"$ & $"$ & $"$ & $"$ & 0.30 & 0.02 & 0.26 & 0.55 & 442.00 & 86.00 & 182.00 & 0.93 & $"$ \\
\hline 156 & Nas-46 & $"$ & $"$ & $"$ & $"$ & 0.23 & 0.02 & 0.15 & 0.62 & 443.00 & 65.00 & 270.00 & 0.88 & $"$ \\
\hline 157 & Nas-47 & $"$ & $"$ & $"$ & $"$ & 0.21 & 0.01 & 0.17 & 0.49 & 435.00 & 81.00 & 233.00 & 0.94 & $"$ \\
\hline 158 & Nas-48 & $"$ & $"$ & $"$ & $"$ & 0.21 & 0.02 & 0.17 & 0.43 & 437.00 & 79.00 & 201.00 & 0.89 & $"$ \\
\hline 159 & Nas-49 & $"$ & $"$ & $"$ & $"$ & 0.35 & 0.02 & 0.39 & 0.52 & 432.00 & 113.00 & 151.00 & 0.95 & $"$ \\
\hline 160 & Nas-50 & $"$ & $"$ & $"$ & $"$ & 0.13 & 0.02 & 0.10 & 0.35 & 444.00 & 78.00 & 273.00 & 0.83 & $"$ \\
\hline 161 & Nas-51 & $"$ & $"$ & $"$ & $"$ & 0.13 & 0.01 & 0.08 & 0.30 & 444.00 & 61.00 & 229.00 & 0.89 & $"$ \\
\hline 162 & Nas-52 & $"$ & $"$ & $"$ & $"$ & 0.33 & 0.06 & 0.39 & 0.48 & 426.00 & 119.00 & 146.00 & 0.87 & $"$ \\
\hline 163 & Nas-53 & $"$ & $"$ & Bima/Sand & $"$ & 52.70 & 20.56 & 297.44 & 10.13 & 427.00 & 564.00 & 19.00 & 0.94 & $"$ \\
\hline 164 & Nas-54 & $"$ & $"$ & $"$ & $"$ & 55.20 & 22.6 & 314.29 & 11.18 & 428.00 & 569.00 & 20.00 & 0.93 & $"$ \\
\hline 165 & Nas-55 & $"$ & $"$ & $"$ & $"$ & 52.10 & 18.10 & 306.91 & 10.87 & 423.00 & 589.00 & 21.00 & 0.94 & $"$ \\
\hline 166 & Nas-56 & $"$ & $"$ & Bima/Shale & $"$ & 0.51 & 0.04 & 0.68 & 0.48 & 425.00 & 134.00 & 94.00 & 0.94 & $"$ \\
\hline 167 & Nas-57 & $"$ & $"$ & $"$ & $"$ & 0.18 & 0.01 & 0.10 & 0.45 & 440.00 & 56.00 & 253.00 & 0.91 & $"$ \\
\hline 168 & Nas-58 & $"$ & $"$ & $"$ & $"$ & 0.30 & 0.01 & 0.21 & 0.37 & 446.00 & 70.00 & 124.00 & 0.95 & $"$ \\
\hline 169 & Nas-59 & $"$ & $"$ & $"$ & $"$ & 0.15 & 0.00 & 0.08 & 0.36 & 444.00 & 54.00 & 242.00 & 1.00 & $"$ \\
\hline 170 & Nas-60 & $"$ & $"$ & $"$ & $"$ & 0.25 & 0.00 & 0.07 & 0.36 & 484.00 & 28.00 & 145.00 & 1.00 & $"$ \\
\hline 171 & Nas-61 & $"$ & $"$ & $"$ & $"$ & 0.21 & 0.00 & 0.08 & 0.38 & 466.00 & 38.00 & 182.00 & 1.00 & $"$ \\
\hline 172 & Nas-62 & $"$ & $"$ & $"$ & $"$ & 0.37 & 0.06 & 0.23 & 0.43 & 456.00 & 62.00 & 116.00 & 0.79 & " \\
\hline 173 & Nas-63 & $"$ & $"$ & $"$ & $"$ & 0.10 & 0.01 & 0.04 & 0.38 & 457.00 & 42.00 & 399.00 & 0.80 & $"$ \\
\hline 174 & Nas-64 & $"$ & " & $"$ & $"$ & 0.29 & 0.00 & 0.06 & 0.30 & 514.00 & 21.00 & 104.00 & 1.00 & $"$ \\
\hline
\end{tabular}


Table 3. TOCs, HIs and Tmax ranges and averages from potential source rocks of the Gongola sub-basin.

\begin{tabular}{|c|c|c|c|c|c|c|c|c|c|c|}
\hline & \multirow{2}{*}{\multicolumn{2}{|c|}{ Age }} & \multirow{2}{*}{\multicolumn{2}{|c|}{ Formation }} & \multicolumn{2}{|c|}{ TOC (Wt\%) } & \multicolumn{2}{|c|}{ HI (mg HC/g TOC) } & \multicolumn{2}{|c|}{$\mathbf{T}_{\max }\left({ }^{\circ} \mathrm{C}\right)$} \\
\hline & & & & & Range & Average & Range & Average & Range & Average \\
\hline \multirow{3}{*}{$\begin{array}{c}\text { Upper } \\
\text { Cretaceous }\end{array}$} & Post-Santonian & Maastrichtian & Gombe & $\begin{array}{c}\text { Shale/Coaly Shale } \\
\text { Facies }\end{array}$ & $0.20-6.84$ & 1.66 & $2-280$ & 45 & \multirow[t]{2}{*}{$282-502$} & \multirow[t]{2}{*}{417} \\
\hline & & & & Shaly Coal Facies & $14.90-23.70$ & 19.60 & $122-178$ & 143 & & \\
\hline & Pre-Santonian & $\begin{array}{c}\text { Upper } \\
\text { Cenomanian-Santonian }\end{array}$ & & Pindiga & $0.04-2.45$ & 0.59 & $5-180$ & 31 & $415-591$ & 420 \\
\hline \multirow{3}{*}{$\begin{array}{c}\text { Lower } \\
\text { Cretaceous }\end{array}$} & & nomanian & & Yolde & $0.30-0.35$ & 0.33 & $26-31$ & 29 & $437-438$ & 438 \\
\hline & \multirow{2}{*}{\multicolumn{2}{|c|}{ Pre-Albian-Aptian }} & \multirow{2}{*}{ Bima } & Shale Facies & $0.10-0.87$ & 0.32 & $21-160$ & 73 & $414-514$ & 444 \\
\hline & & & & Sand Facies & $52.10-55.20$ & 53 & $564-589$ & 574 & $423-428$ & 425 \\
\hline
\end{tabular}

Table 4. TOC and extract compositions of soil stained samples from the Bima Formation in well Nasara-1.

\begin{tabular}{|c|c|c|c|c|c|c|c|c|c|c|c|}
\hline $\begin{array}{l}\text { Sample } \\
\text { Name }\end{array}$ & $\begin{array}{c}\text { Sample } \\
\text { Type }\end{array}$ & Locality & Formation & Lithology & $\begin{array}{c}\text { TOC } \\
(\mathbf{W t} \%)\end{array}$ & $\begin{array}{l}\text { Extract } \\
\text { (mg/mg) }\end{array}$ & $\begin{array}{l}\text { Extract } \\
\text { (ppm) }\end{array}$ & $\begin{array}{c}\text { Extract } \\
\text { (mg/mg TOC) }\end{array}$ & $\begin{array}{l}\text { Saturates } \\
\text { (\%) }\end{array}$ & $\begin{array}{c}\text { Aromatics } \\
\text { (\%) }\end{array}$ & $\begin{array}{c}\text { Hetero-polar } \\
(\%)\end{array}$ \\
\hline NAS54 & $"$ & $"$ & $"$ & $"$ & 55.20 & 237.19 & 237190 & 447.33 & 16.30 & 5.10 & 78.70 \\
\hline NAS55 & $"$ & $"$ & $"$ & $"$ & 52.10 & 187.58 & 187580 & 429.70 & 13.20 & 5.50 & 81.30 \\
\hline NAS56 & $\begin{array}{l}\text { Borehole } \\
\text { Cuttings }\end{array}$ & " & " & Shales & 0.51 & 0.69 & 690 & 360.04 & n.i & n.i & n.i \\
\hline
\end{tabular}

Table 5. Extended hopane distribution of samples from 4710 - $4770 \mathrm{ft}$ in well Nasara-1.

\begin{tabular}{cccccc}
\hline Sample Name & Sample Type & Locality & Formation & Lithology & H31R/H30 \\
\hline NAS 53 & Oil Stained Sand & Nasara-1 Well & Bima & Sands & 0.19 \\
NAS 54 & $"$ & $"$ & & 4 & 0.25 \\
NAS 55 & $"$ & $"$ & 4 & 0.27 \\
\hline
\end{tabular}

Table 6. TOCs, HIs and Tmax ranges and averages from potential source rocks of the Yola sub-basin.

\begin{tabular}{|c|c|c|c|c|c|c|c|c|c|}
\hline & \multirow{2}{*}{ Age } & \multirow{2}{*}{\multicolumn{2}{|c|}{ Formation }} & \multicolumn{2}{|c|}{ TOC (Wt\%) } & \multicolumn{2}{|c|}{ HI (mg HC/g TOC) } & \multicolumn{2}{|c|}{$\mathbf{T}_{\max }\left({ }^{\circ} \mathrm{C}\right)$} \\
\hline & & & & Range & Average & Range & Average & Range & Average \\
\hline \multirow{3}{*}{$\begin{array}{c}\text { Upper } \\
\text { Cretaceous }\end{array}$} & \multirow{3}{*}{ Pre-Santonian } & Coniacian-Santonian & Lamja (Coal) & $51.10-50.70$ & 50.90 & $179-184$ & 182 & 438 & 438 \\
\hline & & Upper Turonian & Jessu & $0.21-0.85$ & 0.52 & $11-49$ & 25 & $431-436$ & 433 \\
\hline & & $\begin{array}{c}\text { Upper } \\
\text { Cenomanian-Turonian }\end{array}$ & Dukul & $0.25-1.15$ & 0.57 & $15-64$ & 33 & $429-442$ & 435 \\
\hline \multirow{2}{*}{$\begin{array}{c}\text { Lower } \\
\text { Cretaceous }\end{array}$} & Cenomanian & Yolde & & $0.10-12.90$ & 2.00 & $27-171$ & 60 & $437-442$ & 439 \\
\hline & Pre-Albian-Aptian & Bima & & - & - & - & - & - & - \\
\hline
\end{tabular}




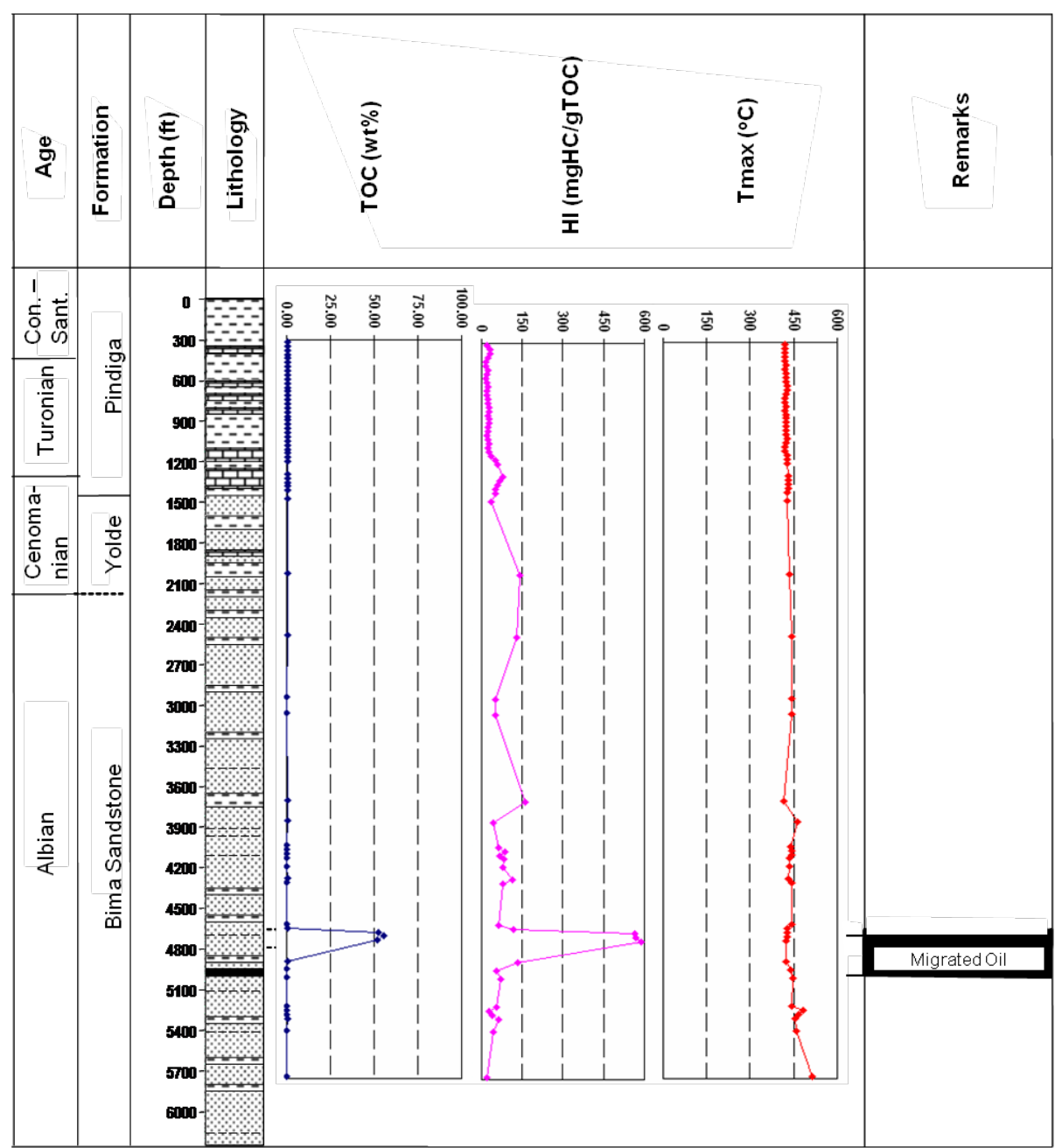

Figure 17. The stratigraphy of the well Nasara-1 showing TOC, HI and Tmax variation with depth and the interval of possible migrated oil.

Potential seal rocks of this system consist locally of the interbedded fluvial (floodplain) and lacustrine shales in the Bima Formation, and interbedded shallow marine and lagoonal shales in the Yolde Formation. The sealing shales within the Yolde Formation are occasionally laterally extensive and may reach thicknesses of up to $4 \mathrm{~m}$. Regional seal constitutes the marine shales of the lower Pindiga Formation in the Gongola Sub-basin and the Dukul Formation in the Yola Sub-basin (Figure 14).

2) The Central Benue Trough

The possible Lower Cretaceous Petroleum System in the Central Benue Trough may constitute the shales and limestones of the marine Albian Asu River Group (Gboko, Uomba and Arufu Formations) as potential source rocks, the sandstones of the Cenomanian Keana and Awe Formations are potential reservoirs while the shales of the basal Ezeaku Formation may act as regional seal [57] (Figure 18).

Organic geochemical data on the potential petroleum source rock (Asu River Group) for this system is very scarce to absent, hence the author could not lay hands on any of the raw data pertaining to organic matter quantity and quality. On maturity however, [57] suggested values in excess of $1.25 \%$ Ro which indicate late gas window stage to over maturity. The Asu River Group may reach an average thickness of up to $1800 \mathrm{~m}$ [57].

The potential reservoir rocks in the Awe and Keana Formations are the flaggy medium-coarse grained calcareous sandstones and the fluvio-deltaic cross-bedded coarse grained feldspathic sandstones respectively. The Awe Formation may reach a thickness of up to $100 \mathrm{~m}$ in places. Although reservoir quality data is not available for these formations, they constitute very important water aquifers around Keana and Awe.

\section{3) The Southern Benue Trough/Anambra Basin}

Data is not available on the existence of the Lower Cretaceous Petroleum System in the Southern Benue Trough/Anambra Basin. Perhaps this may be related to the overall dominance of shale/associated limestone li- 


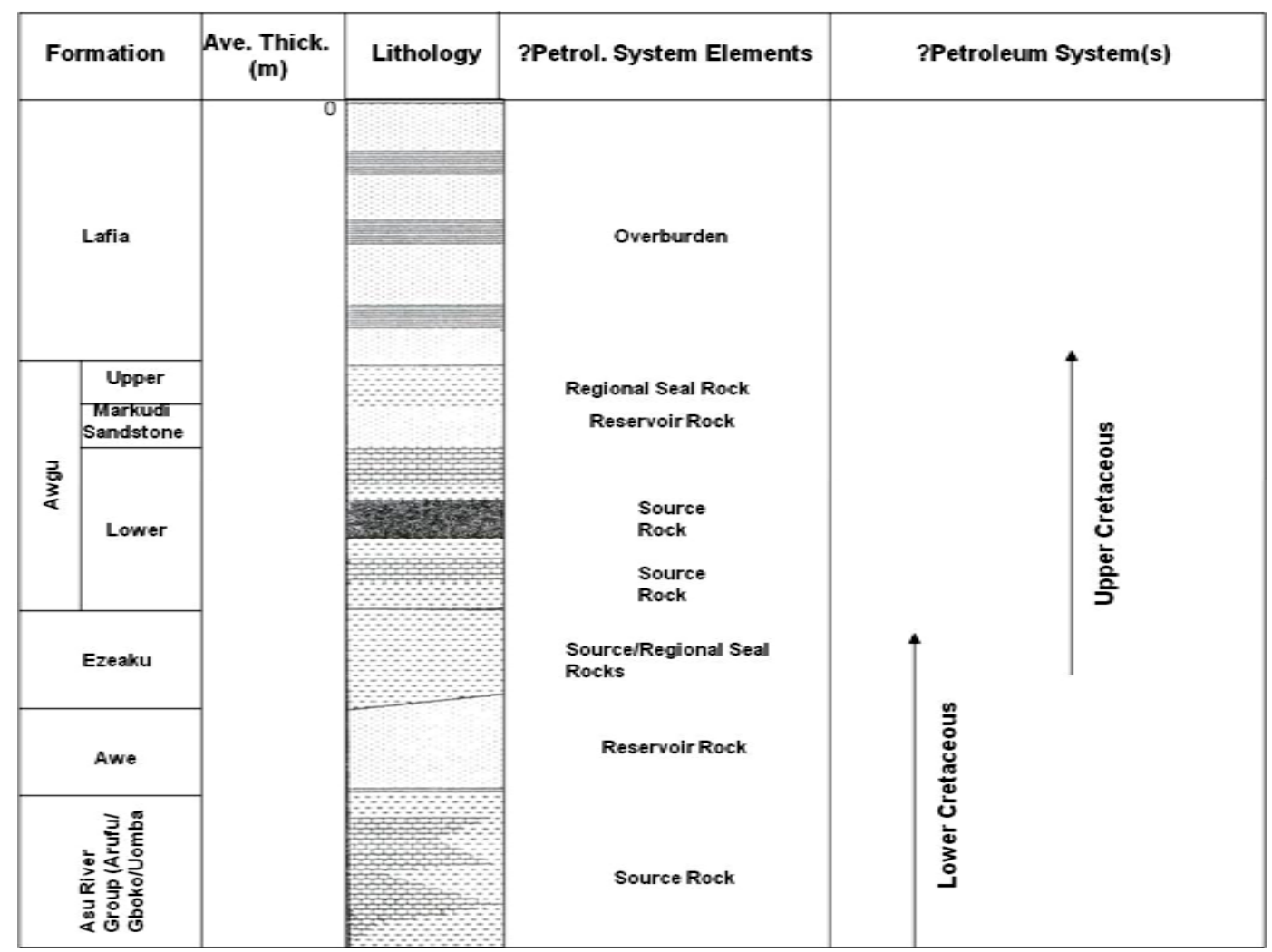

Figure 18. Potential petroleum system(s) in the Central Benue Trough (modified from Obaje et al., 1999).

thologies of the Lower Cretaceous unit (Asu River Group) within the basin (Figure 5). While the shales and limestones of Asu River Group may be potential source rocks, reservoir lithologies seem to be absent.

Although organic geochemical data on the source rock potential of the Asu River Group is not available to us, existing data indicated maturity to overmaturity of the overlying facies of the Ezeaku Group and the Awgu Formation) [60]. This suggests that the underlying Asu River Group most probably has advanced towards the gas kitchen, and consequently had become overcooked.

If Lower Cretaceous Petroleum System exists in the Southern Benue Trough, the shales of the lower Ezeaku Formation will provide a regional seal.

\subsubsection{The Upper Cretaceous Petroleum System}

This system is restricted to the sediments of the Upper Cretaceous Rift Phase II (Figure 7). The lithostratigraphic units formations involved are the Pindiga and Gombe Formations in the Gongola Sub-basin, the Dukul, Jessu, Sekuliye, Numanha and Lamja Formations in the Yola Sub-basin, the Ezeaku Group facies, the Awgu Formation and Nkporo Group facies (including the Enugu Shales and Lafia Formation) in the Southern and Central Benue Trough, and the Coal Measures of the Anambra Basin (e.g. Mamu and Ajali Formations) (Figure 5).

This petroleum system is poorly developed and per- haps non-existent in the Muglad Basin of Sudan due to its little or no source rock potential. It is however well established in the Termit Basin of Niger and Chad Republics [38]. The reservoirs are mainly deltaic-tidal marine clastics (e.g. Sedigi Formation) and fluvial sandstones of Senonian to Maastrichtian age (Table 1) with porosity in the range of $16 \%-25 \%$ (ave. $20 \%$ ) at depth of $2200-3500 \mathrm{~m}$ and permeability of $35-82 \mathrm{md}$ (ave. $52 \mathrm{md}$ ) at same depth interval [38]. These sandstones are of limited thickness and areal extent but may stack up to 60 - $70 \mathrm{~m}$. The Maastrichtian fluvial sandstones may reach up to $400 \mathrm{~m}$ thick and has porosities of $25 \%-35 \%$ [61]. Source rocks are shales of mostly shallow marine to deltaic depositional environment. They are composed of predominantly type III organic matter and have generated oil and gas (Table 1) in the Termit Basin. Average TOCs are in the range of $0.8-1.5 \mathrm{wt} \%$ [61]. Occasionally the TOCs may reach up to $30 \mathrm{wt} \%$ [38], perhaps in coaly facies. The seals are the Upper Cretaceous marine shales, some of which are regional.

1) The Northern Benue Trough

The potential source rocks of this possible petroleum system in the Gongola Sub-basin are shales and limestones of the Pindiga and Fika Formations and perhaps the coals of the Gombe Formation, and the correlative Dukul, Jessu, Sekuliye, Numanha and Lamja Formations in the Yola Sub-basin (Figure 5). 
TOCs from available data in the Yola Sub-basin are in the range of $0.25-1.15 \mathrm{wt} \%$ (ave. $0.57 \mathrm{wt} \%$ ) for the Dukul Formation, $0.21-0.85$ wt\% (ave. 0.52 wt\%) for the Jessu Formation and 51.10 - 50.70 wt\% (ave. 50.90 wt\%) for the coals of the Lamja Formation (Tables 2, 6). HIs from these formations are 15 - $64 \mathrm{mg} \mathrm{HC} / \mathrm{g}$ TOC (ave. $33 \mathrm{mg} \mathrm{HC} / \mathrm{g}$ TOC), 11 - $49 \mathrm{mg} \mathrm{HC/g}$ TOC (ave. 25 mg HC/g TOC) and 179 - 184 mg HC/g TOC (ave. 182 mg HC/g TOC) respectively (Tables 2, 6). These suggest the dominance of type IV OM for the Dukul and Jessu Formations and type II OM for the Lamja Coals.

Available data from the Pindiga Formation of the Gongola Sub-basin indicates 0.04 - 2.45 wt\% TOCs (ave. 0.59 wt\%) with $57.95 \%$ of samples having TOCs of $\geq 0.5$ wt\% (Tables 2, 3). HIs are very low (5 - $180 \mathrm{mg} \mathrm{HC/g}$ TOC) suggesting poor generating potential, except in the upper part of the formation where HIs are mostly above $150 \mathrm{mg} \mathrm{HC/g}$ TOC (Table 2). The upper part suggests oil and gas generating type II organic matter. Shale and coaly shale facies of the Maastrichtian deltaic Gombe Formation show TOC range of 0.20 - 6.87 wt\% (ave. 1.66 wt\%) while the shaly coal facies have TOCs of 14.90 $23.70 \mathrm{wt} \%$ (ave. $19.60 \mathrm{wt} \%$ ) (Tables 2, 3). HIs range from 2 - $280 \mathrm{mg} \mathrm{HC/g}$ TOC with an average of $45 \mathrm{mg}$ $\mathrm{HC} / \mathrm{g}$ TOC in the shale/coaly shale facies and 122 - 178 $\mathrm{mg} \mathrm{HC/g}$ TOC with an average of $143 \mathrm{mg} \mathrm{HC/g}$ TOC in the shaly coal facies (Table 3 ). This suggests that the shaly coal facies are potential source rocks for gas.

The Tmax values of the Upper Cretaceous sediments of the Yola Sub-basin are mostly above the minimum threshold of $435^{\circ} \mathrm{C}$ (Tables 2, 6), hence are generally mature and capable of hydrocarbon generation. The Pindiga and Gombe Formations of the Gongola Sub-basin, on the other hand, show immaturity (Tables 2, 3). The maturity of the Upper Cretaceous sediments in the Yola Sub-basin may be related to the near-by Tertiary volcanic emplacement of the Longuda Plateau. In the western Gongola Sub-basin (Figure 1), the Pindiga and Gombe Formations are overlain by the Kerri-Kerri Formation, hence might have been buried to greater depth to reach maturity.

Possible reservoirs for this system in the Gongola Subbasin are mainly mid-Turonian sandstones of the middle Pindiga Formation and the Gombe Formation (Figure 5). The limestones of the Kanawa Member of the Pindiga Formation may also constitute local reservoirs where individual beds are stacked as in the Ashaka cement quarry (limestones reach thickness of $10 \mathrm{~m}$ ) and where porosities and permeabilities are diagenetically and mechanically enhanced. Generally, the middle members of the Pindiga Formation include moderately well sorted, loosely cemented and thickly developed trough and planar cross-bedded, as well as, hummocky cross-stratified medium to coarse grained sandstones that are occasionally pebbly and graded bedded [53]. Granulestones are also present. These sandstones show coarsening upward cycles at the base, but are fining upward towards the top. The sandstones represent shoreface and tidal/fluvial channels sedimentation at the lower and upper parts of the members respectively [53]. These sandstones may extend for over $10 \mathrm{~km}$ and occur over the entire eastern Gongola Sub-basin. The presence of these members in the sub-cropping part of the western Gongola Sub-basin is possible, but has not been proved. Although porosity and permeability data is lacking, these sandstones constitute excellently reliable aquifers that provide constant supply of a large volume of water needs of the Gombe town from semi-artesian wells at Kwadom. They form also highly productive aquifers in the Kumo area with water yield of $5.80-7.10 \mathrm{l} / \mathrm{sec}$. [62]. The deltaic Gombe Formation, on the other hand, is made up of thickly developed and fairly extensive distributary mouth bars, and distributary and fluvial channel sandstones. These sandstones are moderately well sorted and mostly very fine grained. Porosity and permeability are likely to be highly variable. However, globally the porosities and permeabilities of deltaic sandstone reservoirs range from $11 \%$ 35\% and 250 - 8000 md respectively [63].

In the Yola Sub-basin, siliciclastic reservoir lithologies are scarce except the Coniacian-Santonian deltaic Lamja Formation. This formation may have similar reservoir qualities as the Gombe Formation but is stratigraphically shallow and lacks potential seals. The limestones in the Dukul Formation are thin, hence may not form effective reservoirs.

The Fika Shales could form effective seals for the reservoirs of the middle part of the Pindiga Formation (Figure 14(a)). The potential reservoirs in the Gombe Formation may be sealed by the intercalating silty shales of the formation, but may not be competently and laterally very effective.

2) The Central Benue Trough

Available data on the potential source rocks of the Upper Cretaceous Petroleum System in the central Benue Trough mainly comes from the Turonian-mid-Santonian Awgu Formation [43,57,64,65] (Tables 7, 8).

TOC values from the shales and coaly shales of the Awgu Formation range from 0.43 - 3.90 wt\% (av. 1.40 wt\%), while in the shaly coal and coal facies the TOCs are 14.78 - $79.10 \mathrm{wt} \%$ (av. 45.91 wt\%) (Tables 7, 8). Most of the samples have HIs ranging from 14 - $267 \mathrm{mg}$ $\mathrm{HC} / \mathrm{g}$ TOC with average of $82 \mathrm{mg} \mathrm{HC/g}$ TOC in shale and coaly shale facies and $157 \mathrm{mg} \mathrm{HC} / \mathrm{g}$ TOC in shaly coal and coal facies (Table 8). On the average, the dominant maceral group is vitrinite (Table 9, Figure 19(a)), although some samples are rich in liptinites (Figures 19(b)-(d)). These parameters suggest the predominance of type III (gas-prone) associated with some type II (oil- 
Table 7. Rock-Eval pyrolysis data of samples from the Central Benue Trough $[43,57,65]$.

\begin{tabular}{|c|c|c|c|c|c|c|c|c|c|c|c|c|c|c|}
\hline $\mathrm{S} / \mathrm{N}$ & $\begin{array}{c}\text { Sample } \\
\text { Name }\end{array}$ & Sample Loc. & $\begin{array}{c}\text { Basin/ } \\
\text { Sub-basin }\end{array}$ & $\begin{array}{l}\text { Form./ } \\
\text { Lithol. }\end{array}$ & Age & TOC & S1 & S2 & S3 & Tmax & HI & OI & PI & Source \\
\hline 1 & Awg1 & Obi/Jangwa & CBT & Awgu Coal & $\begin{array}{l}\text { U. Cenom. } \\
\text {-Sant. }\end{array}$ & 64.20 & 7.20 & 116.50 & & 435.00 & 181.00 & & 0.06 & [57] \\
\hline 2 & Awg4 & $"$ & $"$ & $"$ & $"$ & 69.14 & 7.47 & 128.00 & & 437.00 & 185.00 & & 0.06 & $"$ \\
\hline 3 & Awg7 & $"$ & $"$ & $"$ & $"$ & 68.05 & 7.20 & 126.48 & & 437.00 & 186.00 & & 0.05 & $"$ \\
\hline 4 & S502 & $\begin{array}{l}\text { BH120 } \\
\text { Lafia-Obi }\end{array}$ & $"$ & & $"$ & 43.63 & 2.65 & 65.62 & 4.06 & 448.00 & 150.00 & 9.00 & 0.04 & [65] \\
\hline 5 & S503 & $"$ & $"$ & Shale & $"$ & 0.82 & 0.07 & 0.33 & 0.18 & 456.00 & 40.00 & 21.00 & 0.18 & $"$ \\
\hline 6 & S504 & $"$ & $"$ & $"$ & $"$ & 0.80 & 0.18 & 0.29 & 0.10 & 383.00 & 36.00 & 12.00 & 0.38 & $"$ \\
\hline 7 & S508 & $"$ & $"$ & $"$ & $"$ & 0.43 & 0.06 & 0.33 & 0.80 & 455.00 & 76.00 & 186.00 & 0.15 & $"$ \\
\hline 8 & S509 & $"$ & $"$ & Coal & $"$ & 48.39 & 1.89 & 69.31 & 5.51 & 452.00 & 143.00 & 11.00 & 0.03 & $"$ \\
\hline 9 & S510 & $"$ & $"$ & Shale & $"$ & 0.60 & 0.10 & 0.60 & 0.19 & 475.00 & 100.00 & 31.00 & 0.14 & $"$ \\
\hline 10 & S513 & $"$ & $"$ & $"$ & $"$ & 0.97 & 0.09 & 0.88 & 0.23 & 474.00 & 90.00 & 23.00 & 0.09 & " \\
\hline 11 & S514 & $"$ & $"$ & Coal & $"$ & 36.17 & 8.54 & 72.91 & 4.58 & 462.00 & 201.00 & 12.00 & 0.1 & $"$ \\
\hline 12 & S515 & $"$ & $"$ & Shale & $"$ & 0.93 & 0.09 & 0.86 & 0.22 & 483.00 & 92.00 & 23.00 & 0.09 & $"$ \\
\hline 13 & S517 & $"$ & $"$ & $"$ & $"$ & 1.88 & 0.24 & 1.57 & 0.09 & 469.00 & 83.00 & 4.00 & 0.13 & $"$ \\
\hline 14 & S518 & $"$ & $"$ & Coal & $"$ & 18.50 & 1.21 & 15.15 & 3.03 & 453.00 & 81.00 & 16.00 & 0.07 & $"$ \\
\hline 15 & S519 & $"$ & $"$ & Shale & $"$ & 1.34 & 0.14 & 1.02 & 0.32 & 461.00 & 76.00 & 23.00 & 0.12 & $"$ \\
\hline 16 & S533 & $"$ & $"$ & $"$ & $"$ & 0.63 & 0.17 & 0.51 & 0.09 & 468.00 & 80.00 & 63.00 & 0.25 & $"$ \\
\hline 17 & S539 & $"$ & $"$ & $"$ & $"$ & 0.76 & 0.07 & 1.15 & 0.09 & 489.00 & 151.00 & 11.00 & 0.06 & $"$ \\
\hline 18 & S542 & $"$ & $"$ & $"$ & $"$ & 3.90 & 0.36 & 2.83 & 0.48 & 463.00 & 72.00 & 12.00 & 0.11 & $"$ \\
\hline 19 & S550 & $"$ & $"$ & Coal & $"$ & 14.78 & 1.85 & 14.44 & 0.86 & 461.00 & 97.00 & 5.00 & 0.11 & $"$ \\
\hline 20 & S551 & $"$ & $"$ & Shale & $"$ & 2.40 & 0.27 & 2.27 & 0.18 & 493.00 & 93.00 & 7.00 & 0.11 & $"$ \\
\hline 21 & MBJJ1 & Jangwa & $"$ & Shaly Coal & $"$ & 17.40 & 0.08 & 2.49 & 12.49 & 457.00 & 14.00 & 72.00 & 0.03 & [43] \\
\hline 22 & MBJJ2 & $"$ & $"$ & Coal & $"$ & 66.70 & 4.38 & 164.29 & 1.33 & 452.00 & 246.00 & 2.00 & 0.03 & $"$ \\
\hline 23 & MBJJ3 & $"$ & $"$ & Coaly Shale & $"$ & 2.69 & 0.02 & 1.99 & 0.30 & 463.00 & 74.00 & 11.00 & 0.01 & $"$ \\
\hline 24 & MBJJ4 & $"$ & $"$ & Shaly Coal & $"$ & 23.80 & 0.72 & 39.58 & 1.23 & 455.00 & 166.00 & 5.00 & 0.02 & $"$ \\
\hline 25 & MBJJ5 & $"$ & $"$ & Coal & $"$ & 18.50 & 0.38 & 22.18 & 5.32 & 444.00 & 120.00 & 29.00 & 0.02 & $"$ \\
\hline 26 & MBJJ6 & $"$ & $"$ & $"$ & $"$ & 61.10 & 1.93 & 83.05 & 13.60 & 449.00 & 136.00 & 22.00 & 0.02 & $"$ \\
\hline 27 & MBJJ7 & $"$ & $"$ & $"$ & $"$ & 43.10 & 0.19 & 10.81 & 18.12 & 445.00 & 25.00 & 42.00 & 0.02 & $"$ \\
\hline 28 & MBJJ8 & $"$ & $"$ & $"$ & $"$ & 44.20 & 0.26 & 18.42 & 19.13 & 441.00 & 42.00 & 43.00 & 0.01 & $"$ \\
\hline 29 & MBJJ9 & $"$ & $"$ & $"$ & $"$ & 27.00 & 3.93 & 41.20 & 1.65 & 452.00 & 153.00 & 6.00 & 0.09 & $"$ \\
\hline 30 & OBIC2b & $"$ & $"$ & $"$ & $"$ & 70.60 & 2.27 & 171.54 & 2.31 & 453.00 & 243.00 & 3.00 & 0.01 & $"$ \\
\hline 31 & OBIC3 & $"$ & $"$ & $"$ & $"$ & 79.10 & 3.16 & 207.3 & 2.50 & 459.00 & 262.00 & 3.00 & 0.02 & $"$ \\
\hline 32 & ОВIC3b & $"$ & $"$ & $"$ & $"$ & 26.40 & 0.84 & 43.51 & 1.48 & 457.00 & 165.00 & 6.00 & 0.02 & $"$ \\
\hline 33 & OBIC4 & $"$ & $"$ & $"$ & $"$ & 76.30 & 3.04 & 203.84 & 2.52 & 452.00 & 267.00 & 3.00 & 0.01 & $"$ \\
\hline 34 & OBIC5 & $"$ & $"$ & $"$ & $"$ & 75.60 & 2.60 & 192.77 & 2.69 & 457.00 & 225.00 & 4.00 & 0.01 & $"$ \\
\hline 35 & OBIC6 & $"$ & $"$ & Shaly Coal & $"$ & 17.40 & 0.41 & 21.76 & 5.37 & 444.00 & 125.00 & 31.00 & 0.02 & $"$ \\
\hline
\end{tabular}


Table 8. TOCs, HIs and Tmax ranges and averages from potential source rocks of the Central Benue Trough.

\begin{tabular}{|c|c|c|c|c|c|c|c|c|}
\hline \multirow{2}{*}{ Age } & & \multirow{2}{*}{ Formation } & \multicolumn{2}{|c|}{ TOC (wt\%) } & \multicolumn{2}{|c|}{ HI (mg HC/g TOC) } & \multicolumn{2}{|c|}{$\mathrm{T}_{\max }\left({ }^{\circ} \mathrm{C}\right)$} \\
\hline & & & Range & Average & Range & Average & Range & Average \\
\hline \multirow{2}{*}{$\begin{array}{c}\text { Upper Cretaceous } \\
\text { (Pre-Santonian } \\
\text { "Turonian") }\end{array}$} & \multirow[b]{2}{*}{ Awgu } & Shaly Coals \& Coal Facies & $14.78-79.10$ & 45.91 & $14-267$ & 157 & \multirow[b]{2}{*}{$388-489$} & \multirow[b]{2}{*}{455} \\
\hline & & Shales \& Coaly Shales Facies & $0.43-3.90$ & 1.40 & $36-151$ & 82 & & \\
\hline
\end{tabular}

Table 9. Organic petrographic data of samples from the Central Benue Trough [64].

\begin{tabular}{|c|c|c|c|c|c|c|c|c|c|c|c|c|c|}
\hline $\mathbf{S} / \mathbf{N}$ & $\begin{array}{l}\text { Sample } \\
\text { Name }\end{array}$ & $\begin{array}{l}\text { Sample } \\
\text { Loc. }\end{array}$ & $\begin{array}{c}\text { Basin/ } \\
\text { Sub-basin }\end{array}$ & $\begin{array}{l}\text { Form./ } \\
\text { Lithol. }\end{array}$ & Age & Vitrnite & Inertinite & Liptinite & TOTAL & $\begin{array}{c}\% \\
\text { Vitrnite }\end{array}$ & $\begin{array}{c}\% \\
\text { Inertinite }\end{array}$ & $\begin{array}{c}\% \\
\text { Liptinite }\end{array}$ & Source \\
\hline 2 & BH99SN5 & $"$ & " & " & " & 34.60 & 25.70 & 10.40 & 70.70 & 48.94 & 36.35 & 14.71 & $"$ \\
\hline 3 & BH99SN5b & $"$ & " & Awgu/Coal & " & 33.40 & 27.80 & 28.70 & 89.90 & 37.15 & 30.92 & 31.92 & $"$ \\
\hline 4 & BH99SN5c & $"$ & $"$ & $"$ & $"$ & 33.30 & 27.90 & 28.80 & 90.00 & 37.00 & 31.00 & 32.00 & $"$ \\
\hline 5 & BH99SN6 & $"$ & $"$ & $"$ & $"$ & 30.90 & 24.10 & 33.00 & 88.00 & 35.11 & 27.39 & 37.50 & $"$ \\
\hline 6 & BH99SN6b & $"$ & $"$ & $"$ & $"$ & 43.80 & 24.00 & 17.00 & 84.80 & 51.65 & 28.30 & 20.05 & $"$ \\
\hline 8 & BH99SN15 & $"$ & $"$ & $"$ & $"$ & 66.80 & 17.10 & 11.20 & 94.60 & 70.08 & 18.08 & 11.84 & $"$ \\
\hline 9 & BH105SN18 & $"$ & $"$ & $"$ & $"$ & 26.40 & 19.10 & 33.00 & 81.70 & 36.23 & 23.38 & 40.39 & $"$ \\
\hline 10 & BH105SN21b & $"$ & $"$ & $\begin{array}{c}\text { Awgu/ } \\
\text { Shaly Coal }\end{array}$ & " & 11.00 & 47.80 & 6.00 & 64.80 & 16.98 & 73.77 & 9.26 & $"$ \\
\hline 11 & BH105SN28 & $"$ & $"$ & Awgu/Coal & $"$ & 69.80 & 20.70 & 0.00 & 90.50 & 77.13 & 22.87 & 0.00 & $"$ \\
\hline 12 & BH105SN28b & $"$ & $"$ & $"$ & $"$ & 66.70 & 0.00 & 25.30 & 92.00 & 72.50 & 0.00 & 27.50 & $"$ \\
\hline 13 & BH105SN30b & $"$ & $"$ & $\begin{array}{c}\text { Awgu/ } \\
\text { Shaly Coal }\end{array}$ & $"$ & 26.40 & 11.90 & 28.60 & 66.90 & 39.46 & 17.79 & 42.75 & $"$ \\
\hline 15 & BH134SN38 & $"$ & $"$ & Awgu/Shale & $"$ & 26.10 & 6.00 & 33.00 & 65.20 & 40.03 & 9.20 & 50.77 & $"$ \\
\hline 16 & BH134SN39 & $"$ & $"$ & Awgu/Coal & " & 52.90 & 19.30 & 15.80 & 88.00 & 60.11 & 21.93 & 17.95 & $"$ \\
\hline 17 & BH134SN39c & $"$ & $"$ & $"$ & $"$ & 56.80 & 18.00 & 4.40 & 79.20 & 71.72 & 22.73 & 5.56 & $"$ \\
\hline 18 & BH134SN43 & " & $"$ & Awgu/Shale & " & 35.90 & 15.10 & 20.00 & 71.00 & 50.56 & 21.27 & 28.17 & $"$ \\
\hline 19 & BH134SN45b & $"$ & $"$ & Awgu/Coal & $"$ & 67.90 & 9.00 & 8.00 & 84.90 & 79.98 & 10.60 & 9.42 & $"$ \\
\hline 20 & BH136SN48 & $"$ & $"$ & $"$ & $"$ & 52.70 & 25.60 & 14.10 & 92.40 & 57.03 & 27.71 & 15.26 & $"$ \\
\hline 21 & BH136SN48' & $"$ & $"$ & $"$ & $"$ & 56.30 & 20.00 & 13.90 & 90.20 & 62.42 & 22.17 & 15.41 & $"$ \\
\hline 22 & BH136SN52b & $"$ & $"$ & $\begin{array}{c}\text { Awgu/ } \\
\text { Shaly Coal }\end{array}$ & $"$ & 30.20 & 19.70 & 13.10 & 63.00 & 47.94 & 31.17 & 20.79 & $"$ \\
\hline \multirow[t]{2}{*}{23} & BH136SN53 & " & $"$ & $"$ & $"$ & 32.10 & 10.80 & 13.80 & 56.70 & 56.61 & 19.05 & 24.34 & " \\
\hline & & & & & Average & 45.11 & 18.82 & 16.87 & 80.80 & 55.83 & 23.29 & 20.87 & " \\
\hline
\end{tabular}



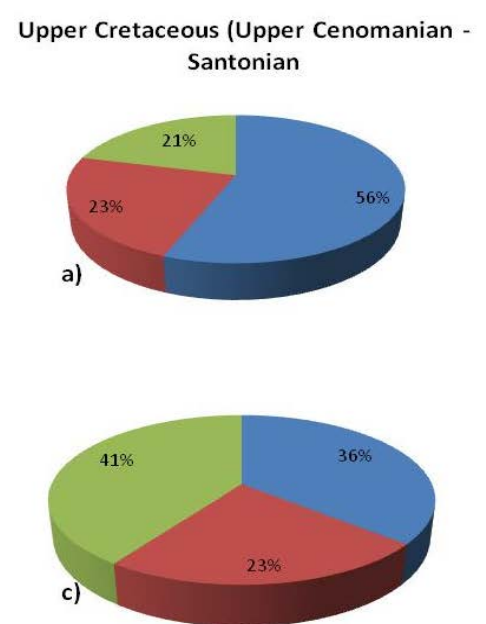

Sample BH105SN18 (Upper Cretaceous)
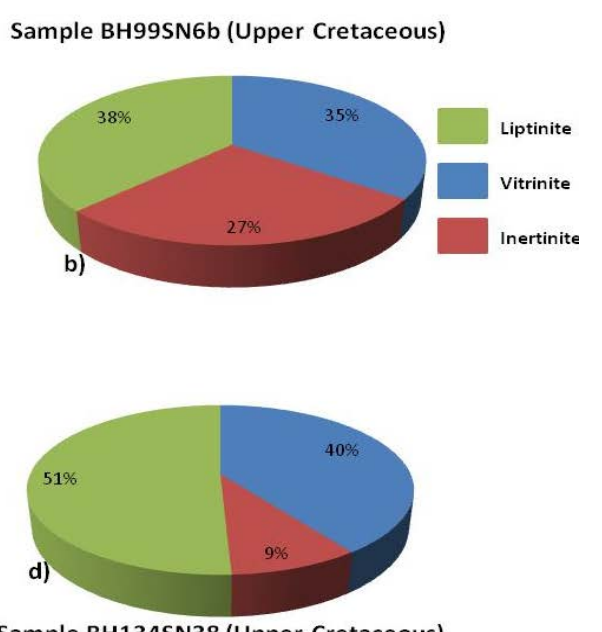

Sample BH134SN38 (Upper Cretaceous)

Figure 19. Maceral group distribution from the Central Benue Trough; (a) is average distribution from the Upper Cretaceous source rock samples.

and gas-prone) organic matter in the Awgu Formation. Therefore predominantly gas may be generated in association of some oil locally. Tmax values for the Awgu Formation range from $388-489^{\circ} \mathrm{C}$ (av. $455^{\circ} \mathrm{C}$, Table 8 ).

These, coupled with the reported $\mathrm{R}_{\mathrm{o}}$ values of $0.76 \%$ $1.25 \%$ [57] suggest that the Awgu Formation is mature and perhaps is in the middle to late oil window. In view of the maturity of the Awgu Formation, oil and predominantly gas might have been generated and expelled in the basin [57].

Potential reservoir rocks for the Upper Cretaceous Petroleum System in the Central Benue Trough could be the Makurdi Sandstone found sandwiched in the Awgu Formation (Figure 18). Keana and Awe Formations could also be reservoirs where structurally juxtaposed against the Awgu Formation. Reservoir quality data of the potential reservoirs is not available.

Potential regional seal for this system in the Central Benue Trough could be the upper shale horizon of the Awgu Formation (Figure 18).

3) The Southern Benue Trough/Anambra Basin

The most viable petroleum system in the southern Benue Trough/Anambra Basin is perhaps the Upper Cretaceous system as observed by [47]. This system may further be subdivided into the pre-Santonian and post-Santonian subsystems. The pre-Santonian subsystem consists of the Ezeaku and Awgu Formations as potential source rocks, the sandy members within the Awgu Formation (e.g. the Coniacian Agbani Sandstone Member) as potential reservoirs, and the basal part of the Nkporo/Enugu Formations as regional seals (Figures 5, 20). The postSantonian subsystem should consist of the shales of the Nkpo-ro/Enugu Formations as major potential source rocks (including the coals and coaly shale of the Mamu and Nsukka Formations), the potential reservoirs consist of sandstones of the Nkporo/Enugu Formations (e.g. the Campanian Owelli and Otobi Sandstone Members), the sandy horizons in the Mamu Formation, the Ajali Sandtone, the sandy horizons of the Nsukka Formation and perhaps the sandstones of the Imo Formation (e.g. the Palaeocene Ebenebe Sandstone Member). Potential regional sealing lithologies could be the shales of the aforementioned potential source rocks and the shale of the Imo Formation within the context of their stratigraphic position vis-à-vis the stratigraphic location of the potential reservoirs (Figure 20).

TOC values range from 0.33 - 7.28 wt\% (ave. 2.52 wt\%) in the pre-Santonian Ezeaku and Awgu Formation with an exceptionally high values of $3-10 \mathrm{wt} \%$ in the Lokpanta Member of the Ezeaku Formation (Table 10). This indicates that the pre-Santonian formations have adequate organic matter quantity for hydrocarbon generation. The HIs range from 38 - $587 \mathrm{mg} \mathrm{HC} / \mathrm{g}$ TOC (ave. $177 \mathrm{mg} \mathrm{HC} / \mathrm{g}$ TOC) for the Ezeaku and Awgu Formations, except again the Lokpanta Member (200 - 600 mg HC/g TOC, Table 11) with values of not less than 200 mg HC/g TOC. In the "mainstream” Ezeaku and Awgu Formations most of the samples have values $\geq 50 \mathrm{mg}$ HC/g TOC but <150 mg HC/g TOC (Tables 10-11) which indicate the predominance of type III (gas-prone) organic matter while the shale of the Lokpanta Member are type I/II (oil- and gas-prone). Tmax values range from $426^{\circ} \mathrm{C}-437^{\circ} \mathrm{C}$ (ave. $431^{\circ} \mathrm{C}$ ) for the "mainstream" Ezeaku and Awgu Formations and $450^{\circ} \mathrm{C}-600^{\circ} \mathrm{C}$ for the Lokpanta Member (Table 11). Although Tmax values of the “mainstream” Ezeaku and Awgu Formations suggest immaturity—marginal maturity, reference [66] observed that the two formations were exposed to burial temperatures in excess of $150^{\circ} \mathrm{C}$ before the advent of the midSantonian tectonic event and perhaps were matured and 
Table 10. Rock-Eval pyrolysis data of samples from the Southern Benue Trough/Anambra Basin [43,72-75].

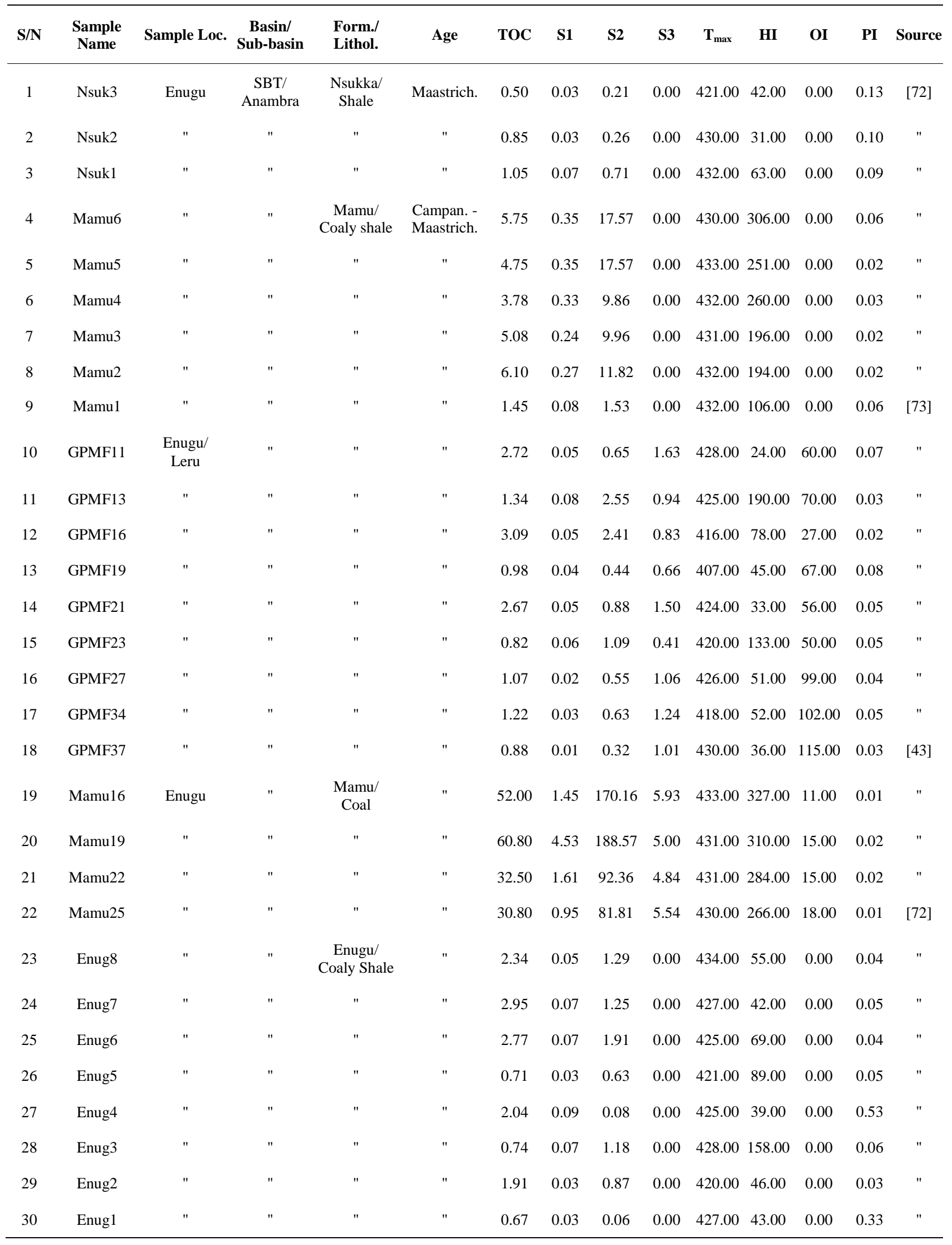




\section{Continued}

\begin{tabular}{|c|c|c|c|c|c|c|c|c|c|c|c|c|c|c|}
\hline 31 & GPES03 & $\begin{array}{l}\text { Enugu/ } \\
\text { Leru }\end{array}$ & $"$ & $\begin{array}{c}\text { Nsukka \& } \\
\text { Enugu/ } \\
\text { Coaly Shale }\end{array}$ & $"$ & 1.16 & 0.06 & 2.54 & 0.58 & 431.00 & 219.00 & 50.00 & 0.02 & [73] \\
\hline 32 & GPES04 & $"$ & $"$ & $"$ & $"$ & 2.69 & 0.04 & 0.94 & 2.42 & 430.00 & 35.00 & 90.00 & 0.04 & $"$ \\
\hline 33 & GPES06 & $"$ & $"$ & $"$ & $"$ & 2.60 & 0.05 & 0.88 & 2.29 & 427.00 & 34.00 & 88.00 & 0.05 & $"$ \\
\hline 34 & GPES07 & $"$ & $"$ & $"$ & $"$ & 0.64 & 0.03 & 0.90 & 0.46 & 429.00 & 140.00 & 72.00 & 0.03 & $"$ \\
\hline 35 & GPES08 & $"$ & $"$ & $"$ & $"$ & 1.57 & 0.01 & 0.47 & 0.77 & 425.00 & 30.00 & 49.00 & 0.02 & $"$ \\
\hline 36 & GPES09 & $"$ & $"$ & $"$ & $"$ & 1.68 & 0.04 & 0.59 & 1.06 & 428.00 & 35.00 & 63.00 & 0.06 & $"$ \\
\hline 37 & GPES29 & $"$ & $"$ & $"$ & $"$ & 3.00 & 0.07 & 3.27 & 0.81 & 430.00 & 109.00 & 27.00 & 0.02 & " \\
\hline 38 & GPES31 & $"$ & $"$ & $"$ & $"$ & 3.32 & 0.09 & 0.70 & 1.89 & 431.00 & 21.00 & 57.00 & 0.11 & $"$ \\
\hline 39 & GPES32 & $"$ & $"$ & $"$ & $"$ & 2.69 & 0.08 & 0.70 & 1.99 & 429.00 & 26.00 & 74.00 & 0.10 & $"$ \\
\hline 40 & Nkpo4 & Leru & $"$ & $\begin{array}{l}\text { Nkporo/ } \\
\text { Coaly Shale }\end{array}$ & $"$ & 2.03 & 0.05 & 0.64 & 0.30 & 423.00 & 32.00 & 15.00 & 0.07 & [43] \\
\hline 41 & Nkpo5 & $"$ & $"$ & $"$ & $"$ & 3.03 & 0.06 & 1.97 & 1.28 & 432.00 & 65.00 & 42.00 & 0.03 & $"$ \\
\hline 42 & Nkpo7 & $"$ & $"$ & Shale & $"$ & 1.57 & 0.02 & 0.35 & 0.28 & 431.00 & 22.00 & 18.00 & 0.05 & $"$ \\
\hline 43 & Nkpo8 & $"$ & $"$ & $"$ & $"$ & 1.35 & 0.02 & 0.30 & 0.27 & 427.00 & 22.00 & 20.00 & 0.06 & $"$ \\
\hline 44 & Enug13 & Enugu & $"$ & $\begin{array}{c}\text { Enugu/ } \\
\text { Coaly Shale }\end{array}$ & $"$ & 3.51 & 0.07 & 1.81 & 1.03 & 426.00 & 327.00 & 15.00 & 0.04 & $"$ \\
\hline 45 & NKP003 & Uturu & $"$ & Nkporo/Shale & $"$ & 0.79 & 0.01 & 0.30 & 0.18 & 428.00 & 38.00 & 28.00 & 0.03 & [74] \\
\hline 46 & NKP004 & $"$ & $"$ & $"$ & $"$ & 1.92 & 0.03 & 0.58 & 0.33 & 431.00 & 30.00 & 17.00 & 0.05 & $"$ \\
\hline 47 & NKP006 & Leru & $"$ & $"$ & $"$ & 2.36 & 0.00 & 0.17 & 0.16 & 429.00 & 7.00 & 7.00 & 0.00 & $"$ \\
\hline 48 & NKP007 & $"$ & $"$ & $"$ & $"$ & 1.02 & 0.04 & 1.25 & 0.23 & 434.00 & 123.00 & 23.00 & 0.03 & $"$ \\
\hline 49 & NKP008 & $"$ & $"$ & $"$ & $"$ & 2.33 & 0.01 & 1.20 & 0.34 & 436.00 & 52.00 & 15.00 & 0.01 & $"$ \\
\hline 50 & NKP009 & $"$ & $"$ & $"$ & $"$ & 1.88 & 0.02 & 0.92 & 0.21 & 431.00 & 49.00 & 11.00 & 0.02 & $"$ \\
\hline 51 & NKP010 & $"$ & $"$ & $"$ & $"$ & 1.53 & 0.01 & 0.42 & 0.21 & 329.00 & 27.00 & 14.00 & 0.02 & $"$ \\
\hline 52 & NKP011 & $"$ & $"$ & $"$ & $"$ & 1.71 & 0.01 & 0.70 & 0.26 & 432.00 & 41.00 & 15.00 & 0.01 & $"$ \\
\hline 53 & NKP012 & $"$ & $"$ & $"$ & $"$ & 1.25 & 0.03 & 0.39 & 0.24 & 430.00 & 31.00 & 19.00 & 0.07 & $"$ \\
\hline 54 & NKP013 & $"$ & $"$ & $"$ & $"$ & 1.93 & 0.03 & 1.51 & 0.19 & 438.00 & 78.00 & 10.00 & 0.02 & $"$ \\
\hline 55 & NKP015 & $"$ & $"$ & $"$ & $"$ & 3.01 & 0.06 & 2.25 & 0.31 & 435.00 & 84.00 & 10.00 & 0.03 & $"$ \\
\hline 56 & NKP016 & $"$ & $"$ & $"$ & $"$ & 2.72 & 0.01 & 1.38 & 0.71 & 443.00 & 51.00 & 26.00 & 0.01 & $"$ \\
\hline 57 & NKP017 & $"$ & $"$ & $"$ & $"$ & 2.83 & 0.08 & 4.34 & 0.24 & 439.00 & 153.00 & 8.00 & 0.02 & $"$ \\
\hline 58 & NKP019 & Amuzo & $"$ & $"$ & $"$ & 1.61 & 0.03 & 0.52 & 0.30 & 428.00 & 32.00 & 19.00 & 0.05 & $"$ \\
\hline 59 & NKP020 & Ihube & $"$ & $"$ & $"$ & 0.54 & 0.01 & 0.11 & 0.14 & 426.00 & 20.00 & 26.00 & 0.08 & $"$ \\
\hline 60 & NKP021 & $"$ & $"$ & $"$ & $"$ & 1.37 & 0.01 & 0.48 & 0.18 & 432.00 & 35.00 & 13.00 & 0.02 & $"$ \\
\hline 61 & CF-7 & $\begin{array}{c}\text { Odukpni } \\
\text { Junction-Itu Roa }\end{array}$ & $\begin{array}{c}\text { SBT/ } \\
\text { Calabar Flank }\end{array}$ & $"$ & $"$ & 0.31 & 0.16 & 0.18 & 0.41 & 430.00 & 58.00 & 132.00 & 0.47 & [75] \\
\hline
\end{tabular}




\begin{tabular}{|c|c|c|c|c|c|c|c|c|c|c|c|c|c|c|}
\hline \multicolumn{15}{|c|}{ Continued } \\
\hline 62 & CF-10 & $\begin{array}{c}\text { Odukpni-Ikom } \\
\text { Road }\end{array}$ & $"$ & $"$ & $"$ & 0.37 & 0.31 & 0.51 & 0.57 & 431.00 & 138.00 & 154.00 & 0.38 & " \\
\hline 63 & CF-4 & $"$ & $"$ & $\begin{array}{l}\text { New Netim } \\
\text { Marl }\end{array}$ & $\begin{array}{l}\text { Cenoman. - } \\
\text { Sant. }\end{array}$ & 0.78 & 0.25 & 0.82 & 1.96 & 436.00 & 105.00 & 251.00 & 0.23 & " \\
\hline 64 & CF-6 & $\begin{array}{c}\text { Odukpni } \\
\text { Junction-Itu Road }\end{array}$ & $"$ & $"$ & $"$ & 0.33 & 0.26 & 0.28 & 0.86 & 430.00 & 85.00 & 261.00 & 0.48 & " \\
\hline 65 & CF-8 & $\begin{array}{c}\text { Asabanga-Abbaiti } \\
\text { Road }\end{array}$ & $"$ & $"$ & $"$ & 1.63 & 0.10 & 0.63 & 0.80 & 426.00 & 39.00 & 49.00 & 0.14 & " \\
\hline 66 & CF-1 & $"$ & $"$ & $\begin{array}{l}\text { Ekenkpon } \\
\text { Shale/Shale }\end{array}$ & $"$ & 5.06 & 2.55 & 29.81 & 0.84 & 428.00 & 589.00 & 17.00 & 0.08 & " \\
\hline 67 & CF-2 & $\begin{array}{l}\text { Odukpni-Ikom } \\
\text { Road }\end{array}$ & $"$ & $"$ & $"$ & 7.28 & 0.37 & 4.77 & 0.38 & 433.00 & 66.00 & 5.00 & 0.07 & " \\
\hline 68 & CF-3 & $"$ & $"$ & $"$ & $"$ & 1.92 & 0.11 & 0.73 & 0.95 & 437.00 & 38.00 & 49.00 & 0.13 & " \\
\hline 69 & CF-5 & $\begin{array}{c}\text { Odukpni } \\
\text { Junction-Itu Road }\end{array}$ & $"$ & $"$ & $"$ & 0.48 & 0.19 & 0.27 & 1.43 & 430.00 & 56.00 & 298.00 & 0.41 & " \\
\hline 70 & CF-9 & $\begin{array}{c}\text { Asabanga-Abbaiti } \\
\text { Road }\end{array}$ & $"$ & $"$ & $"$ & 2.71 & 1.09 & 11.85 & 1.25 & 429.00 & 437.00 & 46.00 & 0.08 & " \\
\hline
\end{tabular}

Table 11. TOCs, HIs and Tmax ranges and averages from potential source rocks of the Southern Benue Trough/Anambra Basin.

\begin{tabular}{|c|c|c|c|c|c|c|c|c|c|c|}
\hline & \multirow{2}{*}{ AGE } & & \multirow{2}{*}{\multicolumn{2}{|c|}{ FORMATION }} & \multicolumn{2}{|c|}{ TOC (Wt\%) } & \multicolumn{2}{|c|}{ HI (mg HC/g TOC) } & \multicolumn{2}{|c|}{$\mathbf{T}_{\text {MAX }}\left({ }^{\circ} \mathrm{C}\right)$} \\
\hline & & & & & Range & Average & Range & Average & Range & Average \\
\hline \multirow{6}{*}{$\begin{array}{l}\text { Upper Cre- } \\
\text { taceous }\end{array}$} & \multirow{4}{*}{ Post-Santonian } & $\begin{array}{c}\text { Upper } \\
\text { Maastrichtian }\end{array}$ & & Nsukka & $0.5-0.05$ & 0.80 & $31-63$ & 45 & $421-432$ & 428 \\
\hline & & & & Coal Facies & $30.80-60.80$ & 40.03 & $266-327$ & 297 & \multirow[b]{2}{*}{$407-433$} & \multirow[b]{2}{*}{428} \\
\hline & & $\begin{array}{l}\text { Lower } \\
\text { Maastrichtian }\end{array}$ & Mamu & Coal Shale Facies & $0.82-6.10$ & 2.78 & $24-306$ & 130 & & \\
\hline & & Campanian & & Enugu/Nkporo & $0.31-3.51$ & 1.86 & $7-327$ & 68 & $420-443$ & 430 \\
\hline & \multirow[b]{2}{*}{ Pre-Santonian } & Upper & \multirow{2}{*}{$\begin{array}{c}\text { Eze-Aku } \\
\text { Group }\end{array}$} & Eze Aku/Awgu & $0.33-7.28$ & 2.52 & $38-589$ & 177 & $426-437$ & 431 \\
\hline & & $\begin{array}{l}\text { Cenomanian- } \\
\text { Santonian }\end{array}$ & & $\begin{array}{l}\text { Lokpanta Member } \\
\text { (Eze Aku) }\end{array}$ & $3.00-10.00$ & - & $200-600$ & - & $450-600$ & - \\
\hline
\end{tabular}

had generated hydrocarbons before the event. The potential source rocks of the post-Santonian formations indicates TOCs of 0.31 - $3.51 \mathrm{wt} \%$ (ave. $1.86 \mathrm{wt} \%$ ), 0.82 6.10 wt\% (ave. 2.78 wt $\%$ ), $30.80-60.80$ wt\% (ave. 40.03 wt\%) and $0.50-0.82$ wt\% (ave. 0.80 wt\%) for the Nkporo/Enugu Formations, coaly shale of the Mamu Formation, coals of the Mamu Formation and Nsukka Formation respectively (Table 11). These indicate adequate organic matter quantity for hydrocarbon generation. The HIs are in the range of $7-327 \mathrm{mg} \mathrm{Hc} / \mathrm{g}$ TOC (ave. $68 \mathrm{mg} \mathrm{HC} / \mathrm{g}$ TOC), 24 - $306 \mathrm{mg}$ HC/g TOC (ave. $130 \mathrm{mg}$ HC/g TOC), 266 - 327 mg HC/g TOC (ave. 297 mg HC/g TOC) and 31 - 63 mg HC/g TOC respectively. Organic petrographic data from the coals of the Mamu and
Nsukka Formations indicates the predominance of vitrinite/huminite [67]. All these parameters indicate predominantly type III with perhaps limited occurrence of type II organic matter. These suggest capability to generate mainly gas on maturity. This deduction was earlier observed by $[47,60,68]$. Generally, the potential source rocks of the post-Santonian petroleum subsystem are immature except perhaps some parts of the Nkporo/ Enugu formations which may be marginally mature (Table 11).

As earlier mentioned, the potential reservoir rocks in the Southern Benue Trough/Anambra Basin are the associated sandstone facies of the predominantly shale lithology of the basin (Figure 20). According to [47] se- 
dimentation in the Anambra Basin was dominantly terrigenous resulting in up to $3000 \mathrm{~m}$ thick shale $(60 \%)$, sands (40\%) and limestone (<1\%). Reference [44] identified three hydrostratigraphic units in the Anambra Basin that includes:

1) Sandy horizons of the Ameki Formation;

2) The Ajali Sandstone, the sandy horizon of the overlying Nsukka Formation, and the upper part of the underlying Mamu Formation;

3) Sandy beds in the Awgu, Nkporo and of the lower Mamu Formations

The first hydrostratigraphic unit is very shallow to form viable reservoir.

References [47,69] inferred that the Mamu and Nsukka Formations are probably delta front sand bars. Ajali Sandstone on the other hand was attributed to fluvial deposition [70] characterized by large channels containing lithic fill of fining upward pebbly sandstones [47]. It is also related to the development of shallow marine subtidal sand bars [69]. These potential reservoir sands are mostly laterally extensive and may reach local thickness of up to $5015 \mathrm{ft}$ (>1000 m) where stacked.

\subsubsection{The Palaeogene Petroleum System}

This system is best developed in the Termit Basin of Niger Republic and the Nigerian Niger Delta where it is related to the Palaeogene Rift Phase III and the Cenozoic-Recent Gulf of Guinea sea water regression. In the Termit Basin, the principal source rocks are lower Eocene shallow marine to paralic shales (200 - 500 m thick) and the middle Eocene lacustrine shales [38]. The lacustrine source rocks are of high quality type I organic matter, derived from fresh water algae and bacteria, and have generated and expelled mainly oil into middle to upper Eocene fluvial channels and lacustrine delta sandstones, as well as, "laterally" into fluvial sandstones of the Palaeocene [38] (Table 1). Oligocene lacustrine shales of up to $1000 \mathrm{~m}$ thick provide regional seal while interbedded shales of the Eocene provide local seal potential [38]. In the Niger Delta the source rocks are marine prodelta shales of the Eocene Akata Formation. The reservoir rocks are mainly the delta front sands of the Oligocene Agbada Formation while the seals are the interbedded shales of the Agbada Formation and the Pliocene-Quaternary Benin Formation [71].

This petroleum system may be absent in the entire Benue Trough/Anambra Basin. In the Gongola Sub-basin of the Northern Benue Trough, sedimentation ceased with the deposition of the Palaeocene continental KerriKerri Formation followed subsequently by the Neogene to Quaternary volcanism (Figure 3). No Cenozoic sedimentation in the Central Benue Trough while the youngest sedimentary unit in the Southern Benue Trough is upper Maastrichtian-Lower Palaeocene Nsukka Formation (Figure 3). The Kerri-Kerri and the Nsukka Formations however, served to bury potential source rocks in the western Gongola Sub-basin and southwestern Anambra Basin respectively to greater depths than elsewhere and therefore have some relevance in terms of enhancing thermal maturity of the sub-cropping Cretaceous sediments [76].

\subsection{Hydrocarbon Traps}

\subsubsection{The Benue Trough}

Traps for hydrocarbons in the Benue Trough are expected to mimic those identified in the WCARS basins of Termit, Doba, Doseo and Muglad. The fact that the Benue Trough shares the same tectonic origin and evolution of initial rifting, thermotectonic sagging, strike-slip faulting, and particularly mid-Santonian and end-Cretaceous compressive phases with the other WCARS basins, also suggests that the structural traps may be of comparable volumes with those in the Termit, Doba, Doseo, etc. Rapid facies changes characterized the stratigraphic successions of the Benue Trough and this suggests the possibility of the presence of stratigraphic traps.

Reference [10] and present author identified E-W trending horsts and grabens (Figure 9) from the upper Aptian and older to lower Cenomanian sedimentary successions in the Gongola Sub-basin of the Northern Benue Trough related to tensional movements and controlled by synrift $\mathrm{N} 60^{\circ} \mathrm{E}$ trending fault system (generally parallel to sub-parallel to the length of the basin). These structures are similar to those reported in the WCARS by [38]. In the Termit Basin and Gongola Sub-basin this pattern is superposed by NNW-SSE trending antithetic faults linked to latest Cenozoic movements.

These types of traps are expected to be dominant in the Lower Cretaceous Petroleum System of the Benue Trough. The block faulting that produced the horst and graben structures can also provide good migration pathways for generated hydrocarbons.

The mid-Santonian and late Maastrichtian compressional events produced additional fracturing and folding that formed traps associated with large compressional anticlines with four-way dip or fault-assisted closures, and listric faults associated with flower structures (e.g. the Lamurde anticline in the Northern Benue Trough, the Keana anticline in the central Benue Trough and the Abakaliki anticline in the southern Benue Trough). These traps may be prevalent in the Upper Cretaceous Petroleum System.

Stratigraphic traps may be in the form of onlap and truncational unconformities, buried channels and, to a lesser extent, pinchouts. 


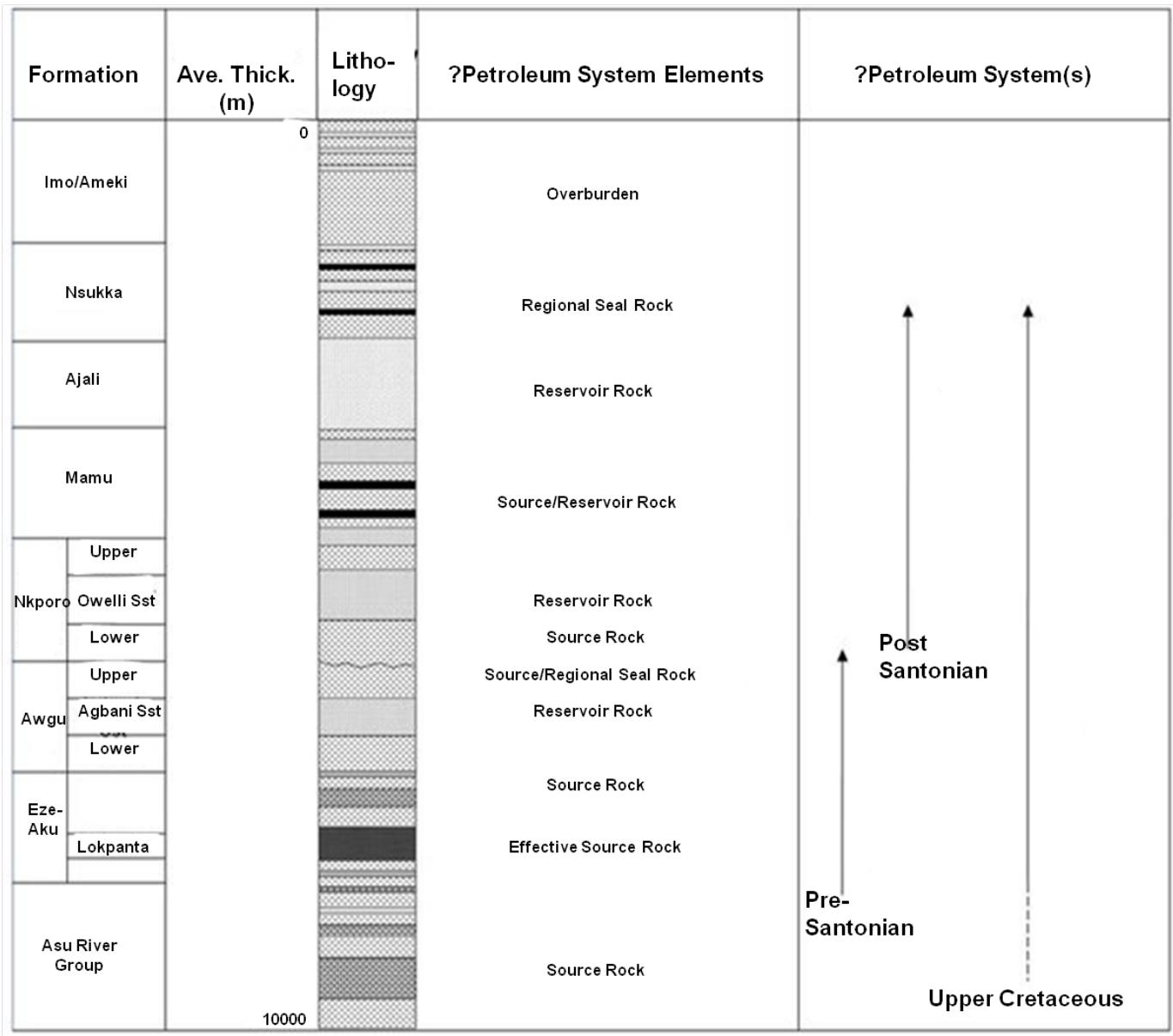

a)

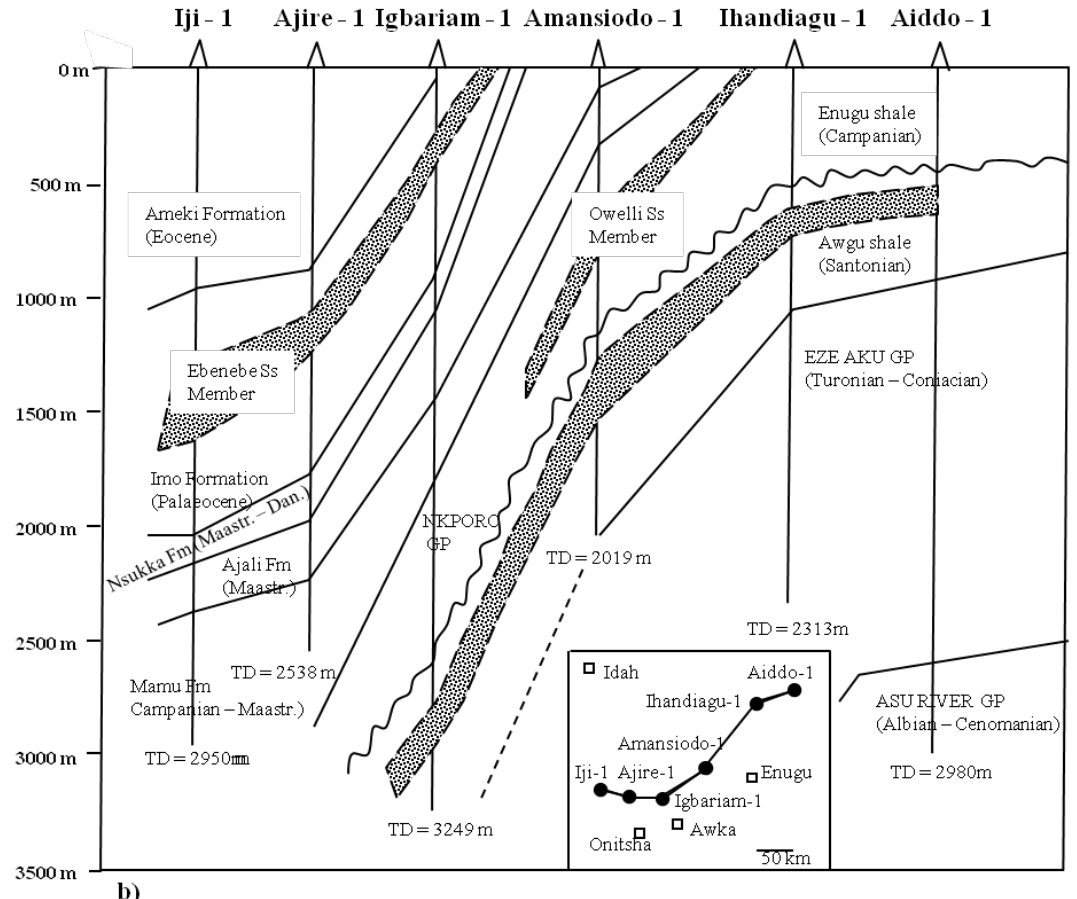

Figure 20. (a) Potential petroleum system(s) in the Southern Benue Trough/Anambra Basin; (b) Subsurface stratigraphy showing relative disposition of potential source, reservoir and seal rocks in the Anambra Basin (from Nwajide, 2005). 


\subsubsection{The Anambra Basin}

Hydrocarbon trapping mechanism in the Anambra Basin is generally the same as in the Benue Trough, except that there is an argument regarding the presence or absence of the end-Maastrichtian tectonic event in the basin. Recently many researchers are accepting the presence of the event in the entire Benue Trough including the Anambra Basin (Dr. Anthony U. Okoro, pers. comm.). Reference [47] observed that the pre-Santonian stage of the Anambra Basin has good to very good trapping structures that include anticlines, faults, unconformities and combination traps most likely related to the mid-Santonian tectonic event; a position earlier taken by [68]. I therefore reject the breaching of these traps by the event that formed them as suggested by [60]. Although there are indications of the breach of traps in the Anambra Basin through surface seepages of hydrocarbon, this might have been caused by later events (e.g. the end-Maastrichtian event). Reference [44] also suggested the presence of post-Maastrichtian event particularly in the Eocene in the Anambra Basin.

Similar structures as above were reported from the post-Santonian stage [44]. Other interesting structures identified on outcrops are growth faults and associated roll over anticlines (Figure 13). The presence of the growth faults was earlier suggested by [68]. Stratigraphic traps in the form of pinch-outs, and buried channels and hills may also be dominant because of the several episodes of transgression/regression in the basin.

\subsection{Petroleum Generation}

\subsubsection{The Benue Trough}

Although SNEPCO has discovered about 33BCF of gas in the well Kolmani River-1 from the Cenomanian Yolde Formation in Gongola Sub-basin, Northern Benue Trough, generally petroleum generation, its timing and expulsion in the Benue Trough are not well known at present. Geothermal gradients in the closely adjacent Bornu Sub-basin however, range from $2.16-5.26^{\circ} \mathrm{C} / 100 \mathrm{~m}$ [77] with the highest values in the region of the Neogene to Quaternary intrusive rocks which generally dominate in the shallow parts (flanks) of the sub-basin [40]. These geothermal gradient values compare well with values of $2.6^{\circ} \mathrm{C}-2.9^{\circ} \mathrm{C} / 100 \mathrm{~m}$ in the Muglad Basin of Sudan [51] and $2.5^{\circ} \mathrm{C}-3.0^{\circ} \mathrm{C} / 100 \mathrm{~m}$ in the basins of Chad and Niger Republics [38]. Modeling for hydrocarbon generation in the Muglad Basin and the basins of Chad and Niger Republics using these geothermal gradients, suggests that at present the oil-generation window lies at $2300-5000 \mathrm{~m}$ depth in the Niger and Chad Republics basins [38], and at $3500-4000 \mathrm{~m}$ in the Muglad Basin [51]. Reference [59] indicated also that the top of "oil window" in east Niger grabens (e.g. the Termit Basin) is located at a depth of between 2200 and $2900 \mathrm{~m}$, and that the top of the "gas window" is between 3600 and $4000 \mathrm{~m}$. These geothermal gradient values and oil-generation windows could be extrapolated for the Benue Trough. The most prospective areas may occur in the axial part of the basins where thicknesses of sedimentary cover are high.

It is worth mentioning at this point, the effect of the end-Cretaceous (end-Maastrichtian) tectonic event and the mid-Santonian/Neogene to Quaternary volcanism visà-vis petroleum generation and preservation in the basins (especially in the Northern Benue Trough). The effect of the end Cretaceous event may be positive by enhancing trapping mechanisms if petroleum generation post-date it and may be negative, on the other hand, if generation pre-date it. The later may open up some of the earlier formed petroleum traps resulting into tertiary migration of the petroleum to high level traps or loss to the surface. The volcanism has also similar effect depending on whether generation occurs pre- or post-volcanism. If volcanism is pre-generation, it may enhance source rock maturity due to increase in heat flow in adjacent areas, but otherwise it may burn up hydrocarbon accumulations that occur close to the volcanic plutons and sills.

\subsubsection{The Anambra Basin}

Several sub-commercial oil and gas discovery were made at different horizons in the Anambra Basin of Nigeria in addition to the heavy crude seepages recorded at Ugwueme (Figure 21) within the Owelli Sandstone of the Nkporo Group. This attests to hydrocarbon generation in the basin. Geothermal gradients in the sandy horizons of the Anambra Basin range from $9.2^{\circ} \mathrm{C}-24^{\circ} \mathrm{C}$ and $29^{\circ} \mathrm{C}$ $70^{\circ} \mathrm{C}$ in shales [47]. These are very high compared to what obtains in the Benue Trough and have made the top of the principal zone of oil generation at the southern parts of the basin to be at a depth of about $1900 \mathrm{~m}$ where temperature of about $60^{\circ} \mathrm{C}$ is inferred [47]. Reference [78] suggested earlier that favourable levels of thermal evolution had been attained and hydrocarbon was generated by the pre-Santonian lithologies (especially the Awgu Formation and facies of the Ezeaku Group) before the advent of the mid-Santonian thermotectonic event.

\section{Conclusions}

From the above review, it can be strongly deduced that at least two potential petroleum systems may be abound in the Benue Trough/Anambra Basin of Nigeria. These petroleum systems are:

1) The Lower Cretaceous Petroleum System that may most likely be both oil and gas generating, and

2) The Upper Cretaceous Petroleum System that is mainly gas generating.

Structures favouring the formation of petroleum traps 


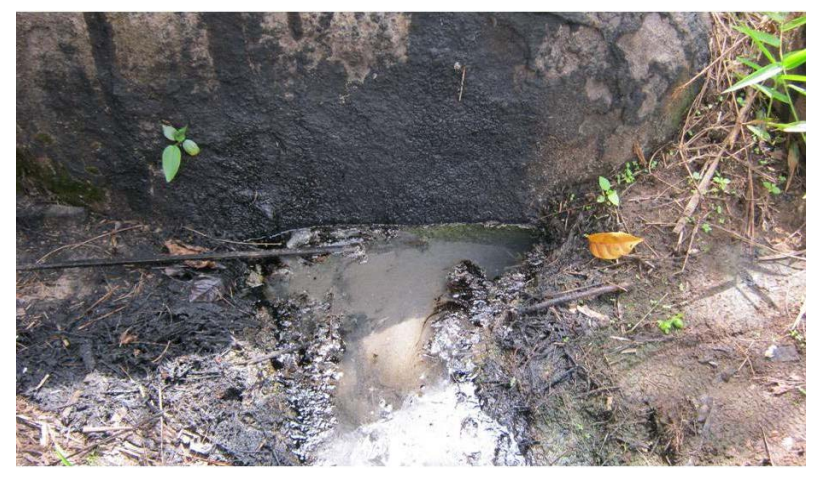

Figure 21. Heavy crude seepage from the Owelli Sandstone at Egwueme, Anambra Basin.

are typical of those related to rift tectonics and include horsts and grabens, large scale transpressional anticlines, inverted fault blocks and drag folds amongst others.

It is recommended that further exploration in the Benue Trough and Anambra Basin should utilize the systemic approach. Bulk compositional (molecular) organic geochemistry method should be used to assess the potential source rocks.

\section{Acknowledgements}

The author is grateful to the National Centre for Petroleum Research and Development of the Energy Commission of Nigeria for the financial support of the project and its permission to publish the work. Dr. Aliyu Jauro of the Abubakar Tafawa Balewa University is also acknowledged for proof reading the draft manuscript and his positive criticisms.

\section{REFERENCES}

[1] BPE, “Nigerian Economy,” Bureau of Public Enterprises Report. 2006. www.bpe.gov.ng.

[2] Wikipedia, "Economy of Nigeria," Wikipedia Foundation Inc., 2006. www.en.wikipedia.org/wiki/Economy_of_Nigeria.

[3] P. Zaborski, F. Ugodulunwa, A. Idornigie, P. Nnabo and K. Ibe, "Stratigraphy and Structure of the Cretaceous Gongola Basin, Northeast Nigeria,” Bulletin du Centre de Recherches elf Exploration Production, Vol. 21, No. 1, 1997, pp. 153-185.

[4] J. Benkhelil, "The Origin and Evolution of the Cretaceous Benue Trough (Nigeria)," Journal of African Earth Science, Vol. 8, No. 2-4, 1989, pp. 251-282. http://dx.doi.org/10.1016/S0899-5362(89)80028-4

[5] L. C. King, "Outline and Distribution of Gondwanaland," Geological Magazine, Vol. 87, No. 5, 1950, pp. 353-359. http://dx.doi.org/10.1017/S0016756800077311

[6] J. D. Carter, W. Barber, E. A. Tait, “The Geology of Part of Adamawa, Bauchi and Bornu Provinces in Northeastern Nigeria," Bulletin-Geological Survey of Nigeria, Vol. 30, 1963, pp. 1-109.
[7] C. R. Cratchley and G. P. Jones, "An Interpretation of the Geology and Gravity Anomalies of the Benue Valley, Nigeria," Overseas Geological Surveys Geophysical Paper, Vol. 1, H.M. Stationery Office, Norwich, 1965, pp. 1-26.

[8] C. R. Cratchley, P. Louis and D. E. Ajakaiye, “Geophysical and Geochemical Evidence for the Benue-Chad Basin Cretaceous Rift Valley System and its Tectonic Implications,” Journal of African Earth Science, Vol. 2, No. 2, 1984, pp. 141-150.

[9] J. Benkhelil and B. Robineau, "Le Fossé de la Bénoué Est-il un Rift?” In: J. Benkhelil, M. Guiraud, J. F. Posard and L. Saugy, Eds., The Bornu-Benue Trough, the Niger Delta and its Offshore: Tectono-Sedimentary Reconstruction during the Cretaceous and Tertiary from Geophysical Data and Geology, In: C. A. Kogbe, Ed., $2^{\text {nd }}$ Edition, Geology of Nigeria, Rock View Nigeria Limited., Jos, 1989, pp. 277-309.

[10] M. Popoff, J. Benkhelil, B. Simon and J. J. Motte, “Approche Géodynamique du Fossé de la Bénoué (N. Nigeria a Partir des Données de Terrain et de Télédètection,” Bulletin Des Centres de Recherches Exploration-Production Elf-Aquitaine, Vol. 7, No. 1, 1983, pp. 323-337.

[11] K. C. Burke, T. F. Dessauvagie and A. J. Whiteman, "Geologic History of the Benue Valley and Adjacent Areas,” In: T. F. J. Dessauvagie and A. J. Whiteman, Eds., African Geology, Ibadan University Press, Ibadan, 1970, pp. 187-205.

[12] N. K. Grant, “The South Atlantic Benue Trough and Gulf of Guinea Cretaceous Triple Junction,” Geological Society of American Bulletin, Vol. 82, No. 8, 1971, pp. 22592298.

http://dx.doi.org/10.1130/0016-7606(1971)82[2295:SAB TAG]2.0.CO;2

[13] M. A. Olade, "Evolution of Nigeria's Benue Trough (Aulacogen): A Tectonic Model,” Geological Magazine, Vol. 112, No. 6, 1975, pp. 575-583. http://dx.doi.org/10.1017/S001675680003898X

[14] J. B. Wright, "Origin of the Benue Trough: A Critical Review,” In: C. A. Kogbe, Ed., Geology of Nigeria, Elisabethan Publ. Co., Lagos, 1976, pp. 309-317.

[15] M. Guiraud, “Tectono-Sedimentary Framework of the Early Cretaceous Continental Bima Formation (Upper Benue Trough, NE Nigeria)," Journal of African Earth Science, Vol. 10, No. 1-2, 1990, pp. 341-353. http://dx.doi.org/10.1016/0899-5362(90)90065-M

[16] O. Lawal and M. Moullade, "Palynological Biostratigraphy of Cretaceous Sediments in the Upper Benue Basin,” Revue de Micropaleontologie, Vol. 29, No. 1, 1986, pp. 61- 83.

[17] M. Guiraud, O. Laborde and H. Philip, "Characterization of Various Types of Deformation and Their Corresponding Deviatoric Stress Tensors using Microfault Analysis," Tectonophysics, Vol. 170, 1989, pp. 1-28.

[18] P. M. Zaborski, "The Cretaceous and Paleocene Transgressions in Nigeria and Niger," Journal of Mining Geology, Vol. 36, No. 2, 2000, pp. 153-173.

[19] P. M. Zaborski, "Guide to the Cretaceous System in the 
Upper Part of the Upper Benue Trough, Northeastern Nigeria,” African Geosciences Review, Vol. 10, No. 1-2, 2003, pp. 13-32.

[20] R. A. Reyment, "Aspects of the Geology of Nigeria," Ibadan University Press, Ibadan, 1965.

[21] C. A. Kogbe, "Palaeogeographic History of Nigeria from Albian Times,” In: C. A. Kogbe, Ed., Geology of Nigeria, Elisabethan Publishing Co., Lagos, 1976, pp. 237-252.

[22] M. E. Offodile, "A Review of the Geology of the Cretaceous of the Benue Valley,” In: C. A. Kogbe, Ed., Geology of Nigeria, Elisabethan Publishing Co., Lagos, 1976, pp. 319-330.

[23] S. W. Petters, "Central West African Cretaceous-Tertiary Benthic Foraminifera and Stratigraphy,” Palaeontolographica Abteilung A, Vol. 179, E. Schweizerbart'sche Verlagsbuchhandlung, 1982, pp. 1-104.

[24] S. W. Petters and C. M. Ekweozor, "Petroleum Geology of Benue Trough and Southeastern Chad Basin," AAPG Bulletin, Vol. 66, No. 8, 1982, pp. 1141-1149.

[25] C. S. Nwajide, "Geology of Nigeria's Sedimentary Basins,” CSS Bookshops Ltd, Lagos, 2013.

[26] M. Rebelle, "The Marine Transgression in the Benue Trough (NE Nigeria): A Paleogeographic Interpretation of the Gongila Formation," Journal of African Earth Science, Vol. 10, No. 4, 1990, pp. 643-655. http://dx.doi.org/10.1016/0899-5362(90)90031-9

[27] T. J. A. Reijers and S. W. Petters, "Sequence Stratigraphy Based on Microfacies Analysis: Mfamosing Limestone, Calabar Flank, Nigeria," Geologie en Mijnbouw, Vol. 76, No. 3, 1997, pp. 197-215. http://dx.doi.org/10.1023/A:1003089529914

[28] P. Allix, E. Grosdidier, S. Jardine, O. Legoux and M. Popoff, “Decouverte d' Aptien Supe'rieur a'Albien Inférieur Date' par Microfossiles dans la Se’rie Détritique Cretace du Fosse de la Benoue (Nigeria),” In: H. Gebhardt, Ed., Journal Paper, Cenomanian to Turonian Foraminifera from Ashaka (NE Nigeria): Quantitative Analysis and Palaeoenvironmental Interpretation, Cretaceous Research, Vol. 18, No. 1, 1997, pp. 17-36. http://dx.doi.org/10.1006/cres.1996.0047

[29] M. Brunet, J. Dejax, A. Brillanceau, J. Congleton, W. Downs, M. Duperon-Laudoueneix, V. Eisenmann, K. Flanagan, L. Flynn, E. Heinzt, J. Hell, L. Jacobs, Y. Jehenne, E. Ndjeng, G. Mouchelin and D. Pilbeam, "Mise en evidence d' une Sédimentations Précoce d'Age Barremien dans lé Fossé de la Bénoué en Afrique Occidentale (Bassin de Mayo Oulo Léré, Cameroun),” In: J. Benkhelil, M. Guiraud, J. F. Posard and L. Saugy, Eds., The BornuBenue Trough, the Niger Delta and its Offshore: Tectono-Sedimentary Reconstruction during the Cretaceous and Tertiary from Geophysical Data and Geology, In: C. A. Kogbe, Ed., $2^{\text {nd }}$ Edition, Geology of Nigeria, Rock View Nigeria Limited., Jos, 1989, pp. 277-309.

[30] R. A. Reyment and R. V. Dingle, "Paleogeography of Africa during the Cretaceous Period," Palaeogeography, $\mathrm{Pa}$ laeoclimatology, Palaeoecology, Vol. 59, 1987, pp. 93116. http://dx.doi.org/10.1016/0031-0182(87)90076-9

[31] S. O. Nwachukwu, "The Tectonic Evolution of the Southern Portion of the Benue Trough, Nigeria,” Geological Maga- zine, Vol. 109, No. 5, 1972, pp. 411-419. http://dx.doi.org/10.1017/S0016756800039790

[32] L. C. Amajor, "The Cenomanian Hiatus in the Southern Benue Trough, Nigeria,” Geological Magazine, Vol. 122, No. 1, 1985, pp. 39-50. http://dx.doi.org/10.1017/S0016756800034063

[33] R. C. Murat, "Stratigraphy and Paleogeography of the Cretaceous and Lower Tertiary in Southern Nigeria," In: T. F. J. Dessauvagie and A. J. Whiteman, Eds., African Geology, Ibadan University Press, Ibadan, 1972, pp. 251266.

[34] P. Allix, "Environments Mésozoïques de la Partie NordOrientale du Fossé de la Bénoué (Nigéria): Stratigraphie, Sédimentologie, Evolution Géodynamique,” In: M. Popoff, J. Wiedmann and I. Klasz, Eds., a Jounal Paper, The Upper Cretaceous Gongila and Pindiga Formations, Northern Nigeria: Subdivisions, Age, Stratigraphic Correlation and Paleogeographic Implications, Eclogae Geologie Helvetica, Vol. 79, No. 2, 1986, pp. 343-363.

[35] N. G. Obaje and H. Hamza, "Biostratigraphy and Source Rock Potential of Cretaceous Sediments in the Upper Benue Trough and Chad Basin of Nigeria,” Department of Geology, Ahmadu Bello University, Zaria, 1997. Unpublished Report.

[36] R. Guiraud and W. Bosworth, "Senonian Basin Inversion and Rejuvenation of Rifting in Africa and Arabia: Synthesis and Implications to Plate-scale Tectonics," Tectonophysics, Vol. 282, No. 1-4, 1997, pp. 39-82. http://dx.doi.org/10.1016/S0040-1951(97)00212-6

[37] K. C. Short and A. J. Stauble, "Outline of Geology of Niger Delta,” AAPG Bulletin, Vol. 51, No. 5, 1967, pp. 761-770.

[38] G. J. Genik, "Petroleum Geology of Cretaceous-Tertiary Rift Basins in Niger, Chad and Central African Republics," AAPG Bulletin, Vol. 77, No. 8, 1993, pp. 1405-1434.

[39] J. Benkhelil, M. Guiraud, J. F. Posard and L. Saugy, “The Bornu-Benue Trough, the Niger Delta and its Offshore: Tectono-Sedimentary Reconstruction during the Cretaceous and Tertiary from Geophysical Data and Geology,” In: C. A. Kogbe, Ed., $2^{\text {nd }}$ Edition, Geology of Nigeria, Rock View Nigeria Limited., Jos, 1989, pp. 277-309.

[40] A. A. Avbovbo, E. O. Ayoola and G. A. Osahon, "Depositional and Structural Styles in Chad Basin of Northeastern Nigeria,” AAPG Bulletin, Vol. 70, No. 12, 1986, pp. 17871798.

[41] N. R. Giedt, "Unity Field Sudan, Muglad Rift Basin, Upper Nile Province,” In: E. A. Beaumont and N. H. Foster, Eds., Structural Traps III: Tectonic Fold and Fault Traps: American Association of Petroleum Geology Bulletin Treatise of Petroleum Geology Atlas of Oil and Gas Fields, 1990, pp. 177-197.

[42] O. S. Adegoke, A. E. Agumanu, M. J. Benkhelil and P. O. Ajayi, "New Stratigraphic, Sedimentologic and Structural Data on the Kerri-Kerri Formation, Bauchi and Borno States, Nigeria," Journal of African Earth Sciences, Vol. 5, No. 3, 1986, pp. 249-277.

[43] N. G. Obaje, H. Wehner, G. Scheeder, M. B. Abubakar and A. Jauro, "Hydrocarbon Prospectivity of Nigeria's Inland Basins: From the Viewpoint of Organic Geoche- 
mistry and Organic Petrology,” AAPG Bulletin, Vol. 88, No. 3, 2004, pp. 325-353.

[44] K. M. Onuoha, “A Close Look at the Petroleum Potential of the Anambra Basin: Inputs from Geophysics and Geohistory,” In: C. O. Okogbue, Ed., Hydrocarbon Potentials of the Anambra Basin: Geology, Geochemistry and Geohistory Perspectives, Great AP Express Publisher Limited, Nsukka, 2005, pp. 83-110.

[45] H. D. Klemme, "Petroleum Basins-Classifications and Descriptions,” Journal of Petroleum Geology, Vol. 3, No. 2, 1980, pp. 187-207. http://dx.doi.org/10.1111/j.1747-5457.1980.tb00982.x

[46] M. B. Abubakar, E. F. C. Dike, N. G. Obaje, H. Wehner and A. Jauro, "Petroleum Prospectivity of Cretaceous Formations in the Gongola Basin, Upper Benue Trough, Nigeria: An Organic Geochemical Perspective on a Migrated Oil Controversy," Journal of Petroleum Geology, Vol. 31, No. 4, 2008, pp. 387-408. http://dx.doi.org/10.1111/j.1747-5457.2008.00428.x

[47] C. S. Nwajide, “Anambra Basin of Nigeria: Synoptic Basin Analysis as a Basis for Evaluating its Hydrocarbon Prospectivity,” In C. O. Okogbue, Ed., Hydrocarbon Potentials of the Anambra Basin: Geology, Geochemistry and Geohistory Perspectives, Great AP Express Publisher Limited, Nsukka, 2005, pp. 83-110.

[48] L. B. Magoon and W. G. Dow, "The Petroleum System," AAPG, Memoir 60, 1994, pp. 3-24.

[49] J. M. Hunt, "Petroleum Geochemistry and Geology," $2^{\text {nd }}$ Edition, Freeman, W.H. and Co., New York, 1996, p. 743

[50] R. C. Haack, P. Sunderaman, J. O. Diedjomahor, H. Xiao, N. J. Gant, E. D. May and K. Kelsch, "Niger Delta Petroleum System, Nigeria,” In: M. R. Mello and B. J. Katz, Eds., Petroleum System of South Atlantic Margins, AAPG Memoir 73, 2000, pp. 213-231.

[51] M. Y. Abdalla, M. J. Pearson, W. A. Ashcroft, J. E. Iliffe and A. J. Whiteman, "Modeling Petroleum Generation in Southern Muglad Rift Basin, Sudan,” AAPG Bulletin, Vol. 83, No. 12, 1999, pp. 1943-1964.

[52] A. A. Mustapha and R. V. Tyson, "Organic Facies of Early Cretaceous Syn-rift Lacustrine Source Rocks from the Muglad Basin, Sudan,” Journal of Petroleum Geology, Vol. 25, No. 3, 2002, pp. 351-366. http://dx.doi.org/10.1111/j.1747-5457.2002.tb00013.x

[53] M. B. Abubakar, "Biostratigraphy, Palaeoenvironment and Organic Geochemistry of the Cretaceous Sequences of the Gongol Basin, Upper Benue Trough, Nigeria,” Ph.D dissertation, Abubakar Tafawa Balewa University, Bauchi, 2006.

[54] N. K. Samaila, "Reservoirs Potentials of the Upper Bima Sandstone in the Yola and Lau-Lamurde Basins, Upper Benue Trough, NE Nigeria,” Ph.D. Dissertation, Abubakar Tafawa Balewa University, Bauchi, 2007.

[55] M. Popoff, "Du Gondwana à l'Atlantique Sud: Connexions du Fossé de la Bénoué Avec les Basins du Nord-est Brésilien Jusqu’à l’Ouverture du Golfe de Guinée au Cretace Inférieur,” Journal of African Earth Science, Vol. 7, No. 2, 1988, pp. 409-431. http://dx.doi.org/10.1016/0899-5362(88)90086-3

[56] M. Guiraud, "Mécanisme de Formation du Basin Crétacé sur décrochements Multiples de la Haute-Bénoué (Nigéria),” Bulletin Des Centres de Recherches Exploration-Production Elf-Aquitaine, Vol. 15, No. 1, 1991, pp. 11-67.

[57] N. G. Obaje, O. K. Ulu and S. W. Petters, "Biostratigraphy and Geochemical Controls of Hydrocarbon Prospects in the Benue Trough and Anambra Basin, Nigeria," NAPE Bulletin, Vol. 14, No. 1, 1999, pp. 18-54.

[58] S. O. Akande, O. J. Ojo, B. D. Erdtmann and M. Hetenyi, "Paleoenvironment, Source Rock Potential and Thermal Maturity of the Upper Benue Rift Basins, Nigeria: Implications for Hydrocarbon Exploration,” Organic Geochemistry, Vol. 29, No. 1-3, 1998, pp. 531-542. http://dx.doi.org/10.1016/S0146-6380(98)00125-9

[59] O. J. Ojo and S. O. Akande, "Petroleum Geochemical Evaluation of the Mid Cretaceous Sequence in the Dadiya Syncline, Yola Basin, Northeastern Nigeria," Journal of Mining and Geology, Vol. 38, No. 1, 2002, pp. 35-42. http://dx.doi.org/10.4314/jmg.v38i1.18772

[60] C. M. Ekweozor, "Searching for Petroleum in the Anambra Basin of Nigeria,” In: C. O. Okogbue, Ed., Hydrocarbon Potentials of the Anambra Basin: Geology, Geochemistry and Geohistory Perspectives, Great AP Express Publication Limited, Nsukka, 2005, pp. 83-110.

[61] M. Zanguina, A. Bruneton and R. Gnnard, “An Introduction to the Petroleum Potential of Niger," Journal of Petroleum Geology, Vol. 21, No. 1, 1998, pp. 83-103. http://dx.doi.org/10.1111/j.1747-5457.1998.tb00647.x

[62] E. F. C. Dike and A. S. Maigari, "Reservoirs of the Northeast and Future of Petroleum Exploration,” Proceedings NCPRD Symposium Sustainable Fossil Fuel and Renewable Energy in the Northeast and Other Related Regions of Nigeria, 2009, pp. 50-62.

[63] D. G. Morse, "Siliciclastic Reservoir Rocks," AAPG, Memoir 60, 1994, pp. 121-140.

[64] N. G. Obaje and H. Hamza, "Liquid Hydrocarbon SourceRock Potential of mid-Cretaceous Coals and Coal Measures in the Middle Benue Trough of Nigeria,” International Journal of Earth Science, Vol. 89, No. 1, 2000, pp. 130-139. http://dx.doi.org/10.1007/s005310050321

[65] A. O. Ehinola, C. M. Ekweozor and B. R. T. Simoneit, "Geological and Geochemical Evaluation of Lafia-Obi Coal, Benue Trough, Nigeria,” NAPE Bulletin, Vol. 15, No. 1, 2000, pp. 92-104.

[66] G. I. Unomah and C. S. Ekweozor, "Application of Vitrinite Reflectance in Reconstruction of Tectonic Features in the Anambra Basin, Nigeria: Implication for Petroleum Potential,” AAPG Bulletin, Vol. 77, No. 3, 1993, pp. 436-451.

[67] M. U. Uzoegbu and E. F. C. Dike, "Petrography and Technological Evaluation of Late Cretaceous Coals from the Anambra basin, Nigeria," Proceedings NCPRD Symposium Sustainable Fossil Fuel and Renewable Energy in the Northeast and Other Related Regions of Nigeria, 2009, pp. 50-62.

[68] A. J. Whiteman, "Nigeria: Its Petroleum Geology, Resources and Potential," Graham and Trotman, London, 1982, p. 394. http://dx.doi.org/10.1007/978-94-009-7361-9

[69] K. O. Ladipo, C. S. Nwajide and S. O. Akande, “Creta- 
ceous and Paleogene Sequences in the Abakaliki and Anambra Basins, Southern Nigeria, A Field Guide,” International Symposium on the Geology of Deltas, Porthacourt, 1992, p. 39.

[70] O. K. Agagu, E. A. Fayose and S. W. Petters, "Stratigraphy and Sedimentation in the Senonian Anambra Basin of Eastern Nigeria," Journal of Mining and Geology, Vol. 22, No. 1-2, 1985, pp. 25-36.

[71] B. D. Evamy, J. Haremboure, P. Kamerling, W. A. Knaap, F. A. Molloy and P. H. Rowlands, "Hydrocarbon Habitat of Tertiary Niger Delta,” AAPG Bulletin, Vol. 62, No. 1, 1978, pp. 1-39.

[72] O. C. Adeigbe and A. E. Salufu, "Geology and petroleum potential of Campano-Maastrichtian sediments in the Anambra Basin, southeastern Nigeria,” NAPE Bulletin, Vol. 22, No. 1, 2010, pp. 13-17.

[73] I. M. Akaegbobi, G. C. Amaechi and O. A. Boboye, "Source Rock Potential and Depositional Environment of the Campanian-Maastrichtian Shales Outcropping along the Enugu-Leru axis, Anambra Basin, Nigeria,” NAPE Bulletin, Vol. 21, No. 1, 2009, pp. 25-37.
[74] A. O. Ehinola, O. O. Sonibare, O. A. Falode and B. O. Awofala, "Hydrocarbon Potentials and Thermal Maturity of Nkporo Shale from Lower Benue Trough, Nigeria,” Journal of Applied Science, Vol. 5, No. 4, 2005, pp. 689695. http://dx.doi.org/10.3923/jas.2005.689.695

[75] A. O. Ehinola, O. O. Sonibare, D. M. Javie and E. A. Oluwale, "Geochemical Appraisal of Organic Matter in the Mid-Cretaceous Sediments of the Calabar Flank, Southeastern, Nigeria," European Journal of Scientific Research, Vol. 23, No. 4, 2008, pp. 567-577.

[76] P. M. Zaborski and M. B. Abubakar, "Evaluation of the Gongola Basin,” Technical Report, NNPC, Abuja, 2010, p. 37.

[77] A. U. Nwaezeapu, "Hydrocarbon Exploration in Frontier Basins: The Nigerian Chad Basin Experience," NMGS Annual Conf., PortHarcourt, 1992, p. 17.

[78] C. M. Ekweozor, "Petroleum Geochemistry Application to Petroleum Exploration in Nigeria's Lower Benue Trough,” Journal of Mining and Geology, Vol. 19, 1982, pp. 122-131. 